\title{
Selective Syntheses of Partially Etherified Derivatives of Tetrakis(2- hydroxyphenyl)ethene. An Alternative to the Calix[4]arene Ligand System
}

Megumi Fujita, Guizhong Qi, Udo H. Verkerk, Trevor L. Dzwiniel, Robert McDonald ${ }^{\dagger}$ and Jeffrey M. Stryker*

\section{Supporting Information}

Instruments and Analysis: IR spectra were obtained on Nicolet Magna IR 750 or Nicolet 20SX spectrophotometer. Nuclear magnetic resonance (NMR) spectra were recorded on Bruker AM-360, AM-400, AM-300, AM-200, and Unity Inova 500. Spectra were obtained at $25{ }^{\circ} \mathrm{C}$ unless otherwise noted. Chemical shifts are reported in parts per million (ppm, $\delta)$ relative to TMS and coupling constants are reported as $J$ in Hz. The "apparent" coupling constants of, for example, a doublet of doublets that appears as a triplet, or broadened signals in which not all couplings are certain, are reported as $J_{o b s}$ or $J$. The assignment of aromatic carbons in ${ }^{13} \mathrm{C}$ NMR spectroscopy is either based on ${ }^{13} \mathrm{C}$-APT spectroscopy or, occasionally, on the relative intensities obtained from broadband decoupled analysis. Mass spectra were obtained on a Kratos MS-50 High Resolution Mass Spectrometer (EI, at 8kV), Micromass VG 7070E (CI, at 6kV), Kratos

MS-9 (FAB at 6kV) or Micromass ZabSpec Sector-TOF (ES, at 4kV). Elemental analyses were performed by the University of Alberta Microanalytical Service Laboratory. Single crystal Xray diffraction analysis was performed by Dr. Robert McDonald of the University of Alberta Department of Chemistry X-ray Crystallography Laboratory.

Reaction Conditions: All air- or moisture-sensitive manipulations were conducted under a nitrogen atmosphere by using standard Schlenk techniques. Flash column chromatographic separations were performed on silica gel 60 (230-400 mesh, SiliCycle). TLC (Silica Gel 60 F254) was visualized by UV light or Morstein reagent. ${ }^{1}$ 
Materials: Unless indicated otherwise, solvents and reagents were purchased from commercial vendors and used as received. The following solvents/liquid reagents were dried and stored as indicated before use: THF, benzene and hexanes were purified by distillation from sodium benzophenone ketyl; toluene was distilled over potassium and degassed; acetonitrile was distilled from $\mathrm{CaH}_{2}$ freshly before use. The solids $\mathrm{KI}$ and $\mathrm{Ni}(\mathrm{OAc})_{2}$ were dried under reduced pressure with vigorous stirring and mild heating overnight and stored in a drybox. $\mathrm{NaH}$ was washed with hexanes to remove the oil, dried in vacuo, and stored in a drybox. The following complexes were prepared by following published procedures: tetrakis(2-methoxyphenyl)ethene, ${ }^{2}$ tetrakis(5-tert-butyl-2-methoxyphenyl)ethene, ${ }^{2}$ tetrakis(2-hydroxyphenyl)ethene $1,{ }^{2}$ 2,2'dimethoxybenzophenone, ${ }^{2}$ and nickel peroxide $\left(\mathrm{NiO}_{2}{ }^{\prime}\right)^{3}$

2-Hydroxy-2'-methoxybenzophenone 3. This reaction is based on a literature procedure. ${ }^{4}$ To a solution of 2,2'-dimethoxybenzophenone $(6.7 \mathrm{~g}, 0.028 \mathrm{~mol})$ in $\mathrm{CH}_{2} \mathrm{Cl}_{2}(95 \mathrm{~mL}$, freshly distilled from $\mathrm{CaH}_{2}$ ) cooled to $-65^{\circ} \mathrm{C}$ under nitrogen atmosphere, was added a solution of $\mathrm{BCl}_{3}$ in heptane $(1.0 \mathrm{M}, 52 \mathrm{~mL}, 0.052 \mathrm{~mol})$. The reaction mixture was allowed to warm to room temperature over $75 \mathrm{~min}$ and then slowly poured into a stirred solution of saturated aqueous $\mathrm{NaHCO}_{3}$ cooled in an ice bath. The organic fraction was extracted into $\mathrm{CH}_{2} \mathrm{Cl}_{2}$ and the extract washed successively with water and saturated $\mathrm{NaCl}$, and then dried over $\mathrm{Na}_{2} \mathrm{SO}_{4}$. After removal of the solvent in vacuo, the residue was almost pure product, containing about $3 \%$ of the starting material (total 6.3 g, 99\%). This material was used without further purification. ${ }^{1} \mathrm{H}$ NMR $\left(360 \mathrm{MHz}, \mathrm{CDCl}_{3}\right): \delta$ $12.18(\sim 0.7 \mathrm{H}, \mathrm{s}, \mathrm{OH}), 7.48(1 \mathrm{H}, \mathrm{ddd}, J=9,7.9,1.9 \mathrm{~Hz}), 7.47(1 \mathrm{H}, \mathrm{ddd}, J=9,7.5,1.9 \mathrm{~Hz}), 7.33$ $(1 \mathrm{H}, \mathrm{dd}, J=7.6,1.9 \mathrm{~Hz}), 7.30(1 \mathrm{H}, \mathrm{dd}, J=7.6,1.9 \mathrm{~Hz}), 7.06(1 \mathrm{H}, \mathrm{td}, J=7.5,1 \mathrm{~Hz}), 7.03(1 \mathrm{H}, \mathrm{dd}$, $J=8.5,1 \mathrm{~Hz}), 7.02(1 \mathrm{H}, \mathrm{d}, \mathrm{br}, J=\sim 8.5 \mathrm{~Hz}), 6.80(1 \mathrm{H}, \mathrm{ddd}, J=\sim 9,7.6,1 \mathrm{~Hz}), 3.78(3 \mathrm{H}, \mathrm{s}, \mathrm{OMe})$, $1.56\left(\sim 0.5 \mathrm{H}, \mathrm{s}, \mathrm{OH}+\mathrm{H}_{2} \mathrm{O} ?\right) .{ }^{13} \mathrm{C} \mathrm{NMR}\left(75 \mathrm{MHz}, \mathrm{CDCl}_{3}, \mathrm{APT}\right): \delta 202.2(\mathrm{C}=\mathrm{O}), 163.0\left(4^{\circ}\right), 156.6$ $\left(4^{\circ}\right), 136.5,133.8,131.9,128.9,127.9\left(4^{\circ}\right), 125.6,120.3\left(4^{\circ}\right), 118.7,118.1,111.5,55.7\left(\mathrm{OCH}_{3}\right)$. 
HRMS (EI) $m / z:$ Calcd for $\mathrm{C}_{14} \mathrm{H}_{12} \mathrm{O}_{3}: 228.07864$. Found: $228.07876(35 \%)\left[\mathrm{M}^{+}\right], 197.06085$ $(100 \%)\left[\mathrm{M}^{+}-\mathrm{OCH}_{3}\right]$

2-Benzyloxy-2'-methoxybenzophenone 4. A mixture of 2-hydroxy-2'-methoxybenzophenone 3 $(8.08 \mathrm{~g}, 0.0354 \mathrm{~mol}), \mathrm{K}_{2} \mathrm{CO}_{3}(24.5 \mathrm{~g}, 0.177 \mathrm{~mol})$ and $\mathrm{BnBr}(4.4 \mathrm{~mL}, 0.037 \mathrm{~mol})$ in $90 \mathrm{~mL}$ of DMSO was stirred at room temperature overnight. The reaction mixture was diluted with diethyl ether and water. The aqueous layer was extracted with additional diethyl ether and the combined organic layers were washed successively with water and saturated $\mathrm{NaCl}$ and then dried over $\mathrm{Na}_{2} \mathrm{SO}_{4}$. After purification by flash chromatography (eluent: $\mathrm{CH}_{2} \mathrm{Cl}_{2} /$ hexanes $=5 / 4 \rightarrow 3 / 2$ ), pure product $(7.98 \mathrm{~g}, 97 \%)$ was obtained as clear, colorless oil. ${ }^{1} \mathrm{H}$ NMR $\left(360 \mathrm{MHz}, \mathrm{CDCl}_{3}\right): \delta 7.61$ $(1 \mathrm{H}, \mathrm{dd}, J=8,1.8 \mathrm{~Hz}), 7.57(1 \mathrm{H}, \mathrm{dd}, J=8,2 \mathrm{~Hz}), 7.43(1 \mathrm{H}, \mathrm{ddd}, J=8.5,7.5,2 \mathrm{~Hz}), 7.39(1 \mathrm{H}$, ddd, $J=8,7.5,1.8 \mathrm{~Hz}), 7.22 \sim 7.18(3 \mathrm{H}$, overlapping), $7.04(1 \mathrm{H}, \mathrm{td}, J=7.5,1 \mathrm{~Hz}), 6.98 \sim 6.94$ (4H, overlapping), $6.84(1 \mathrm{H}, \mathrm{d}, \mathrm{br}, J=8 \mathrm{~Hz}), 5.45\left(2 \mathrm{H}, \mathrm{s}, \mathrm{PhCH}_{2}\right), 3.60\left(3 \mathrm{H}, \mathrm{s}, \mathrm{CH}_{3}\right) .{ }^{13} \mathrm{C} \mathrm{NMR}$ (75 MHz, $\left.\mathrm{CDCl}_{3}, \mathrm{APT}\right): \delta 195.5(\mathrm{C}=\mathrm{O}), 158.4\left(4^{\circ}\right), 157.2\left(4^{\circ}\right), 136.4\left(4^{\circ}\right), 132.6,130.9\left(4^{\circ}\right)$, $130.6\left(4^{\circ}\right), 130.5,130.3,127.5$ (two peaks overlapping), 126.7, 120.8, 120.4, 112.4, 111.7, 70.1 $\left(\mathrm{OCH}_{2} \mathrm{Ph}\right), 55.7\left(\mathrm{OCH}_{3}\right)$. HRMS (EI) $m / z$ : Calcd for $\mathrm{C}_{21} \mathrm{H}_{18} \mathrm{O}_{3}: 318.12558$. Found: 318.12606 $(22 \%)\left[\mathrm{M}^{+}\right], 287.10749(16 \%)\left[\mathrm{M}^{+}-\mathrm{OCH}_{3}\right], 227.07093(8 \%)\left[\mathrm{M}^{+}-\mathrm{C}_{7} \mathrm{H}_{7}\right], 91.05485(100 \%)$ $\left[\mathrm{C}_{7} \mathrm{H}_{7}^{+}\right]$.

2-Benzyloxy-2'-methoxybenzophenone hydrazide. A mixture of 2-benzyloxy-2'-methoxybenzophenone $4(7.98 \mathrm{~g}, 0.025 \mathrm{~mol})$ and hydrazine hydrate $(16.0 \mathrm{~mL}, 0.328 \mathrm{~mol})$ in $28 \mathrm{~mL}$ of butanol was stirred at reflux overnight. The reaction mixture was diluted with water and the organic portion was extracted twice with THF-acetonitrile $(1: 1)$. After washing the organic extracts with saturated $\mathrm{NaCl}$ and drying over $\mathrm{Na}_{2} \mathrm{SO}_{4}$, the volatiles were removed by rotary evaporator to give a mixture of oil and solid. The solid part increased after being left under 
reduced pressure overnight. The resulting crude product weighed more than $>100 \%(8.78 \mathrm{~g})$ and was used without further purification. The product gave a complicated ${ }^{1} \mathrm{H}$ NMR spectrum, presumably a consequence of forming a mixture of syn/anti isomers. HRMS (EI) $m / z$ : Calcd for $\mathrm{C}_{21} \mathrm{H}_{20} \mathrm{~N}_{2} \mathrm{O}_{2}: 332.15247$. Found: $332.15233(16 \%)\left[\mathrm{M}^{+}\right], 316.13307(31 \%)\left[\mathrm{M}^{+}-\mathrm{NH}_{2}\right]$, $301.12286(41 \%)\left[\mathrm{M}^{+}-\mathrm{N}_{2} \mathrm{H}_{3}\right], 91.05483(100 \%)\left[\mathrm{C}_{7} \mathrm{H}_{7}^{+}\right]$.

E-/Z-1,2-Bis(2-benzyloxyphenyl)-bis(2-methoxyphenyl)ethene 5. To a solution of 2Benzyloxy-2'-methoxybenzophenone hydrazide $(5.60 \mathrm{~g}, 0.0168 \mathrm{~mol})$ in acetonitrile $(100 \mathrm{~mL}$, freshly distilled from $\mathrm{CaH}_{2}$ ), protected from moisture by a $\mathrm{CaCl}_{2}$ drying tube and cooled to $0^{\circ} \mathrm{C}$, was added nickel peroxide ("NiO ${ }_{2}$ ", active oxygen atom $2.69 \times 10^{-3} \mathrm{~mol} / \mathrm{g}, 17.3 \mathrm{~g}, 0.0464 \mathrm{~mol}$ ). The reaction mixture was stirred for $40 \mathrm{~min}$ (N.B.: longer reaction times lead to increased formation of byproduct 6 ). The reaction mixture was filtered quickly through basic aluminum oxide (activity I) and the flask and nickel peroxide filter cake were rinsed with fresh acetonitrile (total of $80 \mathrm{~mL}$ ) until the washings were no longer pink. To the deep pink filtrate was added a few drops of a solution of anhydrous $p$-toluenesulfonic acid in benzene; the color immediately changed to yellow-brown. The acidic reaction mixture was neutralized by the addition of a few drops of concentrated aqueous $\mathrm{KOH}$ with stirring, resulting in a lighter yellow color. This neutralization was crucial to avoid the formation of unknown byproducts. After removal of the volatile fraction and purification by flash chromatography (silica gel, eluent $\mathrm{CH}_{2} \mathrm{Cl}_{2} /$ hexanes $=$ $1 / 3 \rightarrow 1 / 1 \rightarrow 4 / 1)$, analytically pure product was obtained (4.68 $\mathrm{g}, 88.5 \%)$ as an inseparable mixture of the diastereoisomers, but these are not distinguishable spectroscopically. $\mathbf{5}(\boldsymbol{E} / \boldsymbol{Z}):{ }^{1} \mathrm{H}$ NMR (360 MHz, $\left.\mathrm{CDCl}_{3}\right): \delta$ 7.25 7.15 (6H, overlapping, br), $7.11(1 \mathrm{H}, \mathrm{d}, \mathrm{br}), 7.03 \sim 6.97(2 \mathrm{H}$, overlapping, br), 6.68 6.59 (4H, overlapping, br), $4.83\left(2 \mathrm{H}, \mathrm{s}, \mathrm{br}, \mathrm{PhCH}_{2}\right), 3.39(3 \mathrm{H}, \mathrm{s}, \mathrm{br}$, $\left.\mathrm{OCH}_{3}\right) .{ }^{13} \mathrm{C} \mathrm{NMR}\left(75 \mathrm{MHz}, \mathrm{CDCl}_{3}, \mathrm{APT}\right.$ and BB): $\delta 157.4\left(4^{\circ}\right), 156.4\left(4^{\circ}\right), 138.0\left(4^{\circ}\right), 137.0$ $\left(4^{\circ}\right), 132.8\left(4^{\circ}\right), 132.4\left(3^{\circ}\right), 132.3\left(3^{\circ}\right), 128.1\left(3^{\circ}\right), 127.34\left(3^{\circ}\right), 127.28\left(3^{\circ}\right), 127.2\left(3^{\circ}\right), 127.1\left(3^{\circ}\right)$, 119.6 $\left(3^{\circ}\right), 111.8\left(3^{\circ}\right), 111.0\left(3^{\circ}\right), 69.7\left(\mathrm{PhCH}_{2}\right), 55.2\left(\mathrm{CH}_{3}\right)$, one $4^{\circ}$ carbon and one $3^{\circ}$ carbon are 
missing or overlap with other aromatic signals. Anal. Calcd for $\mathrm{C}_{42} \mathrm{H}_{36} \mathrm{O}_{4}: \mathrm{C}, 83.42 ; \mathrm{H}, 6.00$. Found: C, 83.24; H, 6.02. HRMS (EI) $m / z$ : Calcd for $\mathrm{C}_{42} \mathrm{H}_{36} \mathrm{O}_{4}$ : 604.26135. Found: 604.26081 $(85 \%)\left[\mathrm{M}^{+}\right], 513.20567(5 \%)\left[\mathrm{M}^{+}-\mathrm{C}_{7} \mathrm{H}_{7}\right], 422.15187(24 \%)\left[\mathrm{M}^{+}-2\left(\mathrm{C}_{7} \mathrm{H}_{7}\right)\right], 91.05485(100 \%)$ $\left[\mathrm{C}_{7} \mathrm{H}_{7}^{+}\right]$. Byproduct 6. ${ }^{1} \mathrm{H}$ NMR $\left(360 \mathrm{MHz}, \mathrm{CDCl}_{3}\right): \delta 7.72(2 \mathrm{H}, \mathrm{dd}$ ?), $7.62(1 \mathrm{H}, \mathrm{dd}, J=\sim 8.5$, $\sim 1 \mathrm{~Hz}$ ) 7.49 7.33 (7H, overlapping), 7.27 (1H, td, $J=\sim 7.5, \sim 1.0 \mathrm{~Hz}), 7.11$ (2H, overlapping?), 3.70 (3H, s, OMe). HRMS (EI) $m / z$ : Calcd for $\mathrm{C}_{21} \mathrm{H}_{16} \mathrm{O}_{2}: 300.11502$. Found: 300.11511 (100\%) $\left[\mathrm{M}^{+}\right]$. The structural assignment of $\mathbf{6}$ is tentative, based on these limited data. ${ }^{5}$

$E-/ Z$-1,2-Bis(2-hydroxyphenyl)-bis(2-methoxyphenyl)ethene (2E, 2Z). [Due to the limited volume of the reaction apparatus, this reaction was performed in two separate batches, with the resulting crude product mixture combined for isolation and purification.] In a small stainless steel autoclave (Parr), a mixture of E-/Z-Bis(2-benzyloxyphenyl)-bis(2-methoxyphenyl)ethene 5 and $10 \% \mathrm{Pd}-\mathrm{C}$ in EtOAc and $\mathrm{MeOH}$ was pressurized with $\mathrm{H}_{2}$ under 400 psig for $17 \mathrm{~h}$. In the first batch, 5 (2.01 g, $3.32 \mathrm{mmol}), 10 \% \mathrm{Pd}-\mathrm{C}$ (0.355 g, $0.334 \mathrm{mmol})$, EtOAc (40 mL), and MeOH $(160 \mathrm{~mL})$ were used. In the second run, 5 (2.48 g, $4.10 \mathrm{mmol}), 10 \% \mathrm{Pd}-\mathrm{C}(0.429 \mathrm{~g}, 0.403$ $\mathrm{mmol})$, EtOAc $(50 \mathrm{~mL})$ and $\mathrm{MeOH}(155 \mathrm{~mL})$ were used. After the hydrogenation was complete, the reaction mixtures were combined, filtered through Celite, and the volatiles removed in vacuo. The crude $E$ - and Z-isomers (ratio: 7/1, as shown by ${ }^{1} \mathrm{H}-\mathrm{NMR}$ spectroscopy) were separated and purified by flash chromatography (hexanes/EtOAc $=20 / 1$, then 10/1). Crystallization of each of the $E$-dominant portion and Z-dominant portion afforded $1.24 \mathrm{~g}(39 \%)$ of pure $E$-isomer $2 \mathbf{E}$ and $0.3817 \mathrm{~g}(12 \%)$ of pure $Z$-isomer $2 Z$. The remaining fractions of $E / Z$ mixture yielded an additional $17 \%$ of product. By use of crystal seeding, considerable additional $\mathbf{2 E}$ or $\mathbf{2 Z}$ could be further crystallized to purity from both the mixture portion and the mother liquors. The stereochemistry of each isomer was assigned based on the structure of $\mathbf{2 Z}$ as determined by Xray crystallography. The crystals of $\mathbf{2 Z}$ used for X-ray analysis were grown from EtOAc/hexanes by slow diffusion. $\boldsymbol{E}$-1,2-Bis(2-hydroxyphenyl)-bis(2-methoxyphenyl)ethene 
2E: IR $\left(\mathrm{CHCl}_{3}\right.$, cast) 3530 (br, s), 3440 (br, s) cm ${ }^{-1} .{ }^{1} \mathrm{H} \mathrm{NMR}\left(360 \mathrm{MHz}, \mathrm{C}_{6} \mathrm{D}_{6}\right): \delta 7.22(2 \mathrm{H}, \mathrm{d}$, br, $J=\sim 7.5 \mathrm{~Hz}), 7.07(2 \mathrm{H}$, br d, $J=7.5 \mathrm{~Hz}), 6.87 \sim 6.81(2 \mathrm{H}+2 \mathrm{H}+2 \mathrm{H}$, overlapping), $6.66(2 \mathrm{H}, \mathrm{td}$, $J=7.5,1 \mathrm{~Hz}), 6.56(2 \mathrm{H}, \mathrm{ddd}, J=\sim 7.5, \sim 7,1.5 \sim 1.8 \mathrm{~Hz}), 6.27(2 \mathrm{H}, \mathrm{dd}, J=\sim 8, \sim 1 \mathrm{~Hz}), 3.21(6 \mathrm{H}$, s, OMe). ${ }^{13} \mathrm{C}$ NMR (75 MHz, acetone- $\mathrm{d}_{6}$, APT and BB): $\delta 156.9\left(4^{\circ}\right), 154.8\left(4^{\circ}\right), 138.2(\mathrm{C}=\mathrm{C})$, $131.5,131.4\left(4^{\circ}\right), 131.1,129.8\left(4^{\circ}\right), 129.1,128.7,121.0,119.5,115.8,111.5,55.8(\mathrm{OMe})$. $\mathrm{UV} / \mathrm{V}$ is $\left(\mathrm{CH}_{2} \mathrm{Cl}_{2}\right): \lambda_{\max }\left(\varepsilon_{\max }\right)=222$ (29000), 284 (13000). Anal. Calcd for $\mathrm{C}_{28} \mathrm{H}_{24} \mathrm{O}_{4}: \mathrm{C}, 79.22 ; \mathrm{H}$, 5.70. Found: C, 78.94; H, 5.73. HRMS (EI) $m / z$ : Calcd for $\mathrm{C}_{28} \mathrm{H}_{24} \mathrm{O}_{4}$ : 424.16745. Found: $424.16699(100 \%)\left[\mathrm{M}^{+}\right]$. Z-1,2-Bis(2-hydroxyphenyl)-bis(2-methoxyphenyl)ethene 2Z: IR $\left(\mathrm{CHCl}_{3}\right.$, cast) 3424 (br, s) cm ${ }^{-1} .{ }^{1} \mathrm{H}$ NMR $\left(360 \mathrm{MHz}, \mathrm{C}_{6} \mathrm{D}_{6}\right): \delta 7.31(2 \mathrm{H}, \mathrm{br} \mathrm{d}, J=\sim 7.5 \mathrm{~Hz}), 7.15$ (2H, dd, overlapping with solvent), $6.91(2 \mathrm{H}, \mathrm{dd}, J=8,1.5 \mathrm{~Hz}), 6.86(2 \mathrm{H}, \mathrm{ddd}, J=\sim 8,7,1.7$ Hz), $6.77(2 \mathrm{H}, \mathrm{ddd}, J=8,7.2,1.8 \mathrm{~Hz}), 6.65(2 \mathrm{H}, \mathrm{td}, J=7.2,1.5 \mathrm{~Hz}), 6.59(2 \mathrm{H}, \mathrm{td}, J=7.5,1 \mathrm{~Hz})$, $6.20(2 \mathrm{H}, \mathrm{dd}, J=8,<1 \mathrm{~Hz}), 3.11(6 \mathrm{H}, \mathrm{OMe}) .{ }^{13} \mathrm{C}$ NMR $(75 \mathrm{MHz}$, acetone-d 6 , APT and BB): $\delta$ $156.9\left(4^{\circ}\right), 154.9\left(4^{\circ}\right), 138.0(\mathrm{C}=\mathrm{C}), 131.9\left(4^{\circ}\right), 131.3,131.2,129.7\left(4^{\circ}\right), 129.0,128.9,120.7$, 119.6, 115.8, 111.4, 55.7 (OMe). UV/Vis $\left(\mathrm{CH}_{2} \mathrm{Cl}_{2}\right): \lambda_{\max }\left(\varepsilon_{\max }\right)=222(29000), 283(13000)$. HRMS (EI) $m / z$ : Calcd for $\mathrm{C}_{28} \mathrm{H}_{24} \mathrm{O}_{4}$ : 424.16745. Found: 424.16685 (100\%) [M+]. Anal. Calcd for $\mathrm{C}_{28} \mathrm{H}_{24} \mathrm{O}_{4}$ : C, 79.22; H, 5.70. Found: C, 78.93; H, 5.50.

Cis-4b,9b-dihydro-4b,9b-bis(2-hydroxyphenyl)benzofuro(3,2-b)benzofuran 8. Tetrakis(2hydroxyphenyl)ethene $1(0.103 \mathrm{~g}, 0.259 \mathrm{mmol})$ and DDQ (2,3-dichloro-5,6-dicyano-1,4benzoquinone, $0.0899 \mathrm{~g}, 0.396 \mathrm{mmol})$ were dissolved in methanol $(15 \mathrm{~mL})$ and stirred at room temperature for $16 \mathrm{~h}$. Reaction progress was followed by TLC (hexane/EtOAc $=1 / 1)$. Upon completion and removal of the volatiles in vacuo, the crude product was separated from the residue by passing it through a short column of silica gel (eluent: hexanes/EtOAc $=1 / 1$ ). Combination of the pure fractions yielded $0.068 \mathrm{~g}(66 \%)$, with additional product present in the impure fractions. Crystals for $\mathrm{X}$-ray analysis were grown by slow evaporation from $\mathrm{Et}_{2} \mathrm{O}$ at room temperature. IR (cast): $3406 \mathrm{~cm}^{-1}(\mathrm{OH}), 1599,1481,1463,749 .{ }^{1} \mathrm{H}$ NMR (400 MHz, 
DMSO-d $\left._{6}, 120^{\circ} \mathrm{C}\right): \delta 8.24(2 \mathrm{H}, \mathrm{br}, \mathrm{OH}), 7.50(2 \mathrm{H}, \mathrm{br}), 7.21(2 \mathrm{H}, \mathrm{ddd}, J=8,7.5,1.5 \mathrm{~Hz}), 7.07$ (2H, d, slightly br, $J=7.5 \mathrm{~Hz}), 6.92(2 \mathrm{H}, \mathrm{d}, J=7.5 \mathrm{~Hz}), 6.89-6.87(2 \mathrm{H}+2 \mathrm{H}$, overlapping, ddd

\& ddd), $6.64\left(2 \mathrm{H}\right.$, dt, slightly br, $\left.J_{o b s}=(7.5), 1 \mathrm{~Hz}\right), 6.35(2 \mathrm{H}, \mathrm{dd}, J=8,1 \mathrm{~Hz}) .{ }^{13} \mathrm{C}$ NMR $(125$ MHz, DMSO-d $\left.6,100^{\circ} \mathrm{C}\right): \delta 158.3\left(4^{\circ}\right), 153.9\left(4^{\circ}\right), 130.4\left(4^{\circ}\right), 129.2,127.9,127.7$ (br), 124.6 (br), $124.0\left(4^{\circ}\right), 120.0,116.7,114.7,109.3$; one $4^{\circ}$ carbon was not observed. $\mathrm{UV} / \mathrm{V}$ is $\left(\mathrm{CH}_{3} \mathrm{OH}\right): \lambda_{\max }$ $\left(\varepsilon_{\max }\right)=279$ (10941), 220 (obscured by solvent band). HRMS (EI) $m / z$ Calcd for $\mathrm{C}_{26} \mathrm{H}_{18} \mathrm{O}_{4}$ : 394.12051. Found: 394.12035 (100\%) [ $\left.\mathrm{M}^{+}\right]$. Anal. Calcd for $\mathrm{C}_{26} \mathrm{H}_{18} \mathrm{O}_{4}: \mathrm{C}, 79.17 ; \mathrm{H}, 4.60$. Found: C, 79.09; H, 4.59 .

Cis-4b,9b-dihydro-4b,9b-bis(2-methoxyphenyl)benzofuro(3,2-b)benzofuran. A mixture of benzofuran 8 (0.206 g, $0.522 \mathrm{mmol}), \mathrm{CH}_{3} \mathrm{I}(1 \mathrm{~mL}, 16 \mathrm{mmol})$ and $\mathrm{K}_{2} \mathrm{CO}_{3}(2.19 \mathrm{~g}, 15.8 \mathrm{mmol})$ in acetone $(45 \mathrm{~mL})$ was stirred under reflux. The reaction was followed by $\mathrm{TLC}($ hexane/EtOAc $=$ 5/1) and after $16 \mathrm{~h}$, a second aliquot of $\mathrm{CH}_{3} \mathrm{I}(1 \mathrm{~mL}, 16 \mathrm{mmol})$ was added. After an additional 26 $\mathrm{h}$, the mixture was cooled to room temperature, the volatiles were removed in vacuo. The resulting crude reaction mixture was diluted with water, and the organic contents were extracted with EtOAc. The organic layer was washed successively with water and brine and dried over $\mathrm{Na}_{2} \mathrm{SO}_{4}$. After removal of the volatiles, pure product was obtained by crystallization from EtOAc/hexanes (0.196 g, 89\%). ${ }^{1} \mathrm{H}$ NMR (360 MHz, $\left.\mathrm{CDCl}_{3}\right)$ : most peaks slightly broadened; $\delta$ $8.01(2 \mathrm{H}, \mathrm{d}, J=7.5 \mathrm{~Hz}), 7.19\left(2 \mathrm{H}, \mathrm{t}, J_{o b s}=7.5 \mathrm{~Hz}\right), 7.07 \sim 7.06(2 \mathrm{H}+2 \mathrm{H}), 6.95 \sim 6.94(2 \mathrm{H}+2 \mathrm{H})$, $6.82\left(2 \mathrm{H}, \mathrm{t}, J_{o b s}=7.5 \mathrm{~Hz}\right), 6.33(2 \mathrm{H}, \mathrm{d}, J=8 \mathrm{~Hz}), 3.96(6 \mathrm{H}, \mathrm{s})$; some unassigned broad peaks were also present. ${ }^{13} \mathrm{C} \mathrm{NMR}\left(75 \mathrm{MHz}, \mathrm{CDCl}_{3}\right): \delta 158.8\left(4^{\circ}\right), 155.7\left(4^{\circ}\right), 131.3\left(4^{\circ}\right), 129.6,128.7$, $128.1\left(4^{\circ}\right), 127.3,124.6,120.7,119.0,110.3,109.8,54.2$. One $4^{\circ}$ carbon was not found in the spectrum. HRMS (EI) m/z Calcd for $\mathrm{C}_{28} \mathrm{H}_{22} \mathrm{O}_{4}$ : 422.15179. Found: 422.15158 (100\%) $\left[\mathrm{M}^{+}\right]$. Anal. Calcd for $\mathrm{C}_{28} \mathrm{H}_{22} \mathrm{O}_{4}$ : C, 79.60; H, 5.25. Found: C, 79.70; H, 5.21. 
Z-Bis(2-hydroxyphenyl)-bis(2-methoxyphenyl)ethane $2 Z$. Method B. To a cooled $\left(-78^{\circ} \mathrm{C}\right)$ solution of tetrakis(2-methoxyphenyl)ethene (0.5 g, $1.1 \mathrm{mmol})$ in $\mathrm{CH}_{2} \mathrm{Cl}_{2}(100 \mathrm{~mL}), \mathrm{BBr}_{3}(1.1$ mmol in 1.1. $\mathrm{mL} \mathrm{CH}_{2} \mathrm{Cl}_{2}$ ) was added slowly. After maintaining at $-78{ }^{\circ} \mathrm{C}$ for $1 \mathrm{~h}$, the reaction mixture was allowed to warm slowly to room temperature and stir for $12 \mathrm{~h}$. The reaction mixture was quenched by addition of saturated $\mathrm{NaHCO}_{3}(20 \mathrm{ml})$ and the mixture was extracted several times with $\mathrm{Et}_{2} \mathrm{O}$. The combined ether extracts were concentrated under reduced pressure and precipitated upon addition of pentane. Pure product $(0.35 \mathrm{~g}, 75 \%)$, spectroscopically identical to authentic $2 Z$, was obtained by flash chromatography on silica, eluting with $20 \%$ ethyl acetate/hexane.

\section{Z-Bis(5- tert-butyl-2-hydroxyphenyl)-bis(5- tert-butyl-2-methoxyphenyl)ethene 9Z. To a} cooled $\left(-78^{\circ} \mathrm{C}\right)$ solution of tetrakis(5-tert-butyl-2-methoxyphenyl)ethene ${ }^{2}(2.0 \mathrm{~g}, 2.9 \mathrm{mmol})$ in $\mathrm{CH}_{2} \mathrm{Cl}_{2}(200 \mathrm{~mL}), \mathrm{BBr}_{3}\left(2.9 \mathrm{mmol}\right.$, in $\left.2.9 \mathrm{~mL} \mathrm{CH}_{2} \mathrm{Cl}_{2}\right)$ was added slowly. After maintaining at $78^{\circ} \mathrm{C}$ for $1 \mathrm{~h}$, the reaction mixture was allowed to warm slowly to room temperature and stir for $12 \mathrm{~h}$. The reaction was quenched by addition of saturated $\mathrm{NaHCO}_{3}(40 \mathrm{ml})$ and the mixture was extracted several times with $\mathrm{Et}_{2} \mathrm{O}$. Pure product (1.34 g, 70\%) was obtained by flash chromatography on silica, eluting with $12 \%$ ethyl acetate/hexane. IR $\left(\mathrm{CH}_{2} \mathrm{Cl}_{2}\right.$ cast, $\left.\mathrm{cm}^{-1}\right) 3423$ (br); ${ }^{1} \mathrm{H}$ NMR (400 MHz, $\mathrm{CDCl}_{3}$ ): $\delta 7.13$ (d, 2H, J = 2.5 Hz), 7.03 7.06 (m, 4H, overlapping), $6.94(\mathrm{dd}, 2 \mathrm{H}, \mathrm{J}=8.5,2.5 \mathrm{~Hz}), 6.58(\mathrm{dd}, 4 \mathrm{H}, \mathrm{J}=8.4,7.2 \mathrm{~Hz}), 6.41(\mathrm{~s}, \mathrm{br}, 2 \mathrm{H}, \mathrm{OH}), 3.65$ (s, 6H, OMe), 1.14 (s, 18H, t-Bu), 1.12 (s, $18 \mathrm{H}, \mathrm{t}-\mathrm{Bu}) .{ }^{13} \mathrm{C}\left(125 \mathrm{MHz}, \mathrm{CDCl}_{3}, \mathrm{APT}\right): \delta 152.9\left(4^{\circ}\right), 150.9$ $\left(4^{\circ}\right), 143.1\left(4^{\circ}\right), 141.9\left(4^{\circ}\right), 138.7\left(4^{\circ}\right), 129.2\left(4^{\circ}\right), 127.7\left(4^{\circ}\right), 127.2\left(3^{\circ}\right), 126.6\left(3^{\circ}\right), 124.9\left(3^{\circ}\right)$, $114.7\left(3^{\circ}\right), 109.8\left(3^{\circ}\right), 94.4\left(3^{\circ}\right), 55.5\left(1^{\circ}\right), 33.8\left(4^{\circ}\right), 31.3\left(1^{\circ}\right)$. HRMS (EI) $m / z:$ Calcd for $\mathrm{C}_{44} \mathrm{H}_{56} \mathrm{O}_{4}: 648.41785$. Found: $648.41818(100 \%)\left[\mathrm{M}^{+}\right]$. Scanned ${ }^{1} \mathrm{H}$ and ${ }^{13} \mathrm{C}-\mathrm{APT}$ NMR spectra are provided as an appendix. 


\section{Mono-Sodium Salt of Z-Bis(5-tert-butyl-2-hydroxyphenyl)-bis(5-tert-butyl-2-methoxy-}

phenyl)ethene. To a solution of Z-bis(5-tert-butyl-2-hydroxyphenyl)-bis(2-methoxyphenyl)ethene $9 \mathrm{Z}(0.077 \mathrm{~g}, 0.118 \mathrm{mmol})$ in THF (3 mL) was slowly added a suspension of $\mathrm{NaH}(0.006$ $\mathrm{g}, 0.25 \mathrm{mmol})$ in THF (2 mL). After maintaining at room temperature for $5 \mathrm{~h}$, the reaction mixture was filtered through Celite, concentrated in vacuo, and layered with hexanes. Slow diffusion gave colorless crystals $(0.023 \mathrm{~g}, 27 \%)$, which were characterized by X-ray crystallography. Elemental analysis (nonstoichiometric THF solvate): Calcd for $\mathrm{C}_{49.5} \mathrm{H}_{66.5} \mathrm{NaO}_{5}$ : C, 77.7\%; H, 8.7\%. Found: C, 76.2\%; H, 8.8\%.

(5-tert-Butyl-2-hydroxyphenyl)-tris(5-tert-butyl-2-methoxyphenyl)ethene 10. To a solution of tetra(5-tert-butyl-2-methoxyphenyl)ethene ${ }^{2}(2.0 \mathrm{~g}, 3.0 \mathrm{mmol})$ in $\mathrm{CH}_{2} \mathrm{Cl}_{2}(150 \mathrm{~mL})$ at $0{ }^{\circ} \mathrm{C}$ under a nitrogen atmosphere was added iodotrimethylsilane $(0.43 \mathrm{~mL}, 3.0 \mathrm{mmol})$. After $1 \mathrm{~h}, \mathrm{CHCl}_{3}(10$ $\mathrm{mL}$ ) was added and the reaction mixture was allowed to stir overnight. The solvent was removed in vacuo and the residue purified by flash chromatography on silica (eluent: $8-10 \%$ THF in pentane) to give pure (5-tert-butyl-2-hydroxyphenyl)-tris(5-tert-butyl-2-methoxyphenyl)ethene

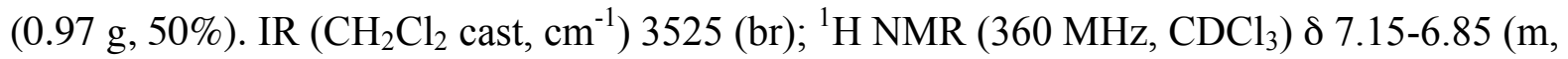
overlapping signals), 6.61-6.5 (m, overlapping signals), 6.26 (br s, OH), 3.56 (s, 3H, OMe), 3.55 (s, 3H, OMe), 3.52 (s, 3H, OMe), 1.14(s, 9H, tert-Bu), 1.12 (s, 9H, tert-Bu), 1.10 (s, 9H, tert-Bu), $1.09\left(\mathrm{~s}, 9 \mathrm{H}\right.$, tert-Bu). ${ }^{13} \mathrm{C} \mathrm{NMR}\left(\mathrm{APT}, 125 \mathrm{MHz}, \mathrm{CDCl}_{3}\right) \delta: 154.9\left(4^{\circ}\right), 154.8\left(4^{\circ}\right), 151.2\left(4^{\circ}\right)$, $142.7\left(4^{\circ}\right), 141.9\left(4^{\circ}\right), 141.1\left(4^{\circ}\right), 135.2\left(4^{\circ}\right), 130.7\left(4^{\circ}\right), 129.9\left(3^{\circ}\right), 127.9\left(3^{\circ}\right), 124.3\left(3^{\circ}\right), 124.2$ $\left(3^{\circ}\right), 123.8\left(3^{\circ}\right), 123.6\left(3^{\circ}\right), 114.0\left(3^{\circ}\right), 110.4\left(3^{\circ}\right), 110.2\left(3^{\circ}\right), 110.1\left(3^{\circ}\right), 55.7\left(1^{\circ}\right), 55.6\left(1^{\circ}\right), 55.5$ $\left(1^{\circ}\right), 33.8\left(4^{\circ}\right), 31.5\left(1^{\circ}\right), 31.4\left(1^{\circ}\right)$. Several signals unobserved or overlapping. $\mathrm{MS} \mathrm{m} / \mathrm{z}$ calculated for $\mathrm{C}_{45} \mathrm{H}_{58} \mathrm{O}_{4}$ : 662.43353; found: 662.43453 (100\%). Scanned ${ }^{1} \mathrm{H}$ and ${ }^{13} \mathrm{C}-\mathrm{APT}$ NMR spectra are provided in an appendix.

Initial fractions from the column provide near quantitative recovery of unconverted starting material. Higher yields of mono-alcohol $\mathbf{1 0}$ can be obtained on a smaller scale by using excess 
iodotrimethylsilane ( 3 equiv) and carefully monitoring the reaction progress by TLC analysis. This procedure, however, also results in the formation of minor amounts of isomeric diols $9 \boldsymbol{E} / \boldsymbol{Z}$ as well as recovered starting material.

\section{Notes and references.}

1. Composition of the Morstein visualization reagent: $20 \mathrm{~g}$ of ammonium molybdate, $0.4 \mathrm{~g}$ of cerium (IV) sulfate, and $500 \mathrm{~mL}$ of $10 \% \mathrm{H}_{2} \mathrm{SO}_{4}$. See: Eur. J. Org. Chem. 2000, 1745-1758.

2. Verkerk, U.; Fujita, M.; Dzwiniel, T. L.; McDonald, R.; Stryker, J. M. J. Am. Chem. Soc. 2002, 124, 9988-9989.

3. Nakagawa, K.; Konaka, R.; Nakata, T. J. Org. Chem. 1962, 27, 1597-1601.

4. Dean, M. F.; Goodchild, J.; Houghton, L. E.; Martin, J. A.; Morton, R. B.; Parton, B.; Price, A. W.; Somvicien, N. Tetrahedron Lett. 1966, 4153-4159.

5. Sonoda, T.; Kobayashi, S.; Taniguchi, H. Bull. Chem. Soc. Jpn. 1976, 49, 2560-2566. 
Appendix 1. Z-Bis(5- tert-butyl-2-hydroxyphenyl)-bis(5-tert-butyl-2-methoxyphenyl)ethene 9Z. ${ }^{1} \mathrm{H}$ NMR spectrum.

$400 \mathrm{MHz} 1 \mathrm{D}$ in $\operatorname{CDC}_{3} 3$ (ref. to $\operatorname{CDC} 13$ e $7.26 \mathrm{ppm}$ ), temp $26.8 \mathrm{C} \rightarrow$ actual temp $=27.0 \mathrm{C}$, asw400 probe

Pulse Sequence: s2pul date:Mar 232004 seqfil:s2pul hz/mm:16.67 sweep width[Hz]:4002 spectrometer:i400 file:exp

Z-1,2-bis(5'-tert-butoxy-2'-hydroxyphenyl)-bis(5'-tert-butoxy-2'-methoxyphenyl)ethene $9 Z$

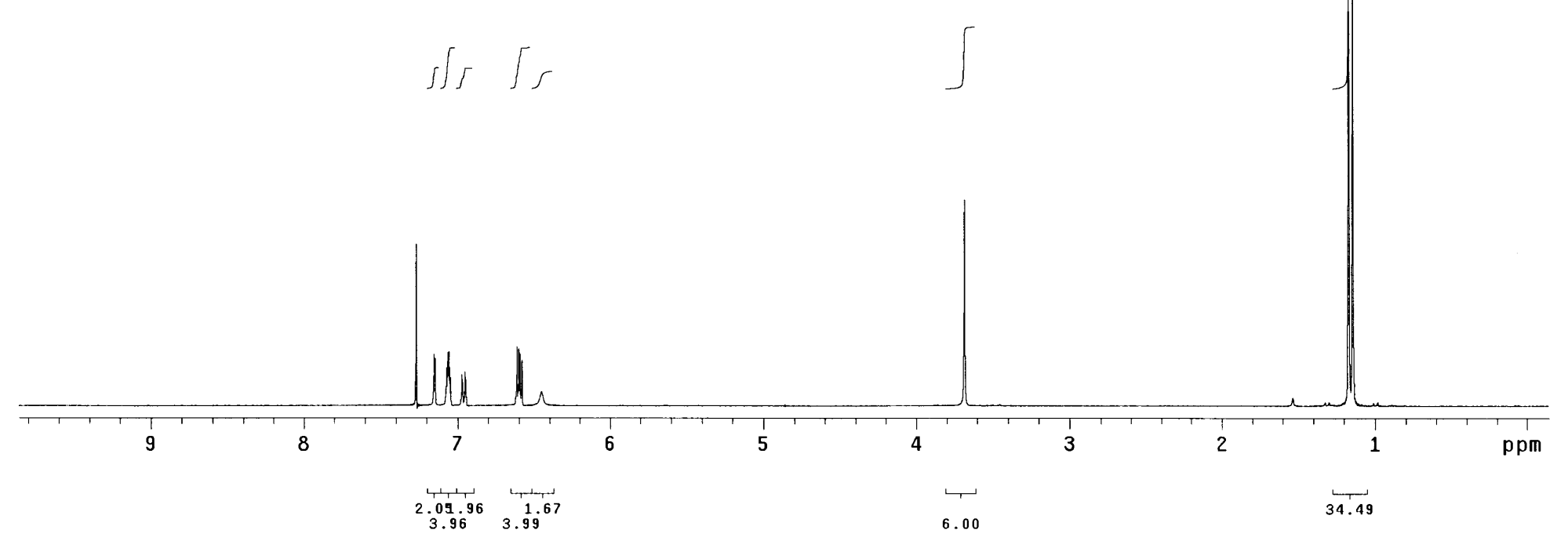


Appendix 1. Z-Bis(5- tert-butyl-2-hydroxyphenyl)-bis(5-tert-butyl-2-methoxyphenyl)ethene 9Z. ${ }^{13} \mathrm{C}$-APT NMR spectrum.

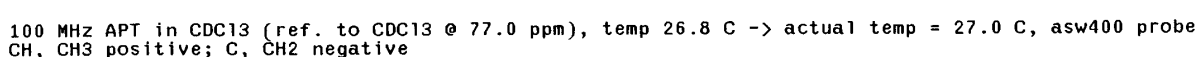
$\begin{array}{lllll}\text { date:Jan } 242003 & \text { seqfil:apt } & \mathrm{hz} / \mathrm{mm}: 83.33 & \text { sweep width[Hz]:20000 } & \text { spectrometer:i400 file:exp } \\ \text { acq.time[s]:2.0 } & \text { relax. time[s]:0.5 } & \text { dig.res.[Hz/pt]:0.31 } & \text { of scans:1168 } & \end{array}$

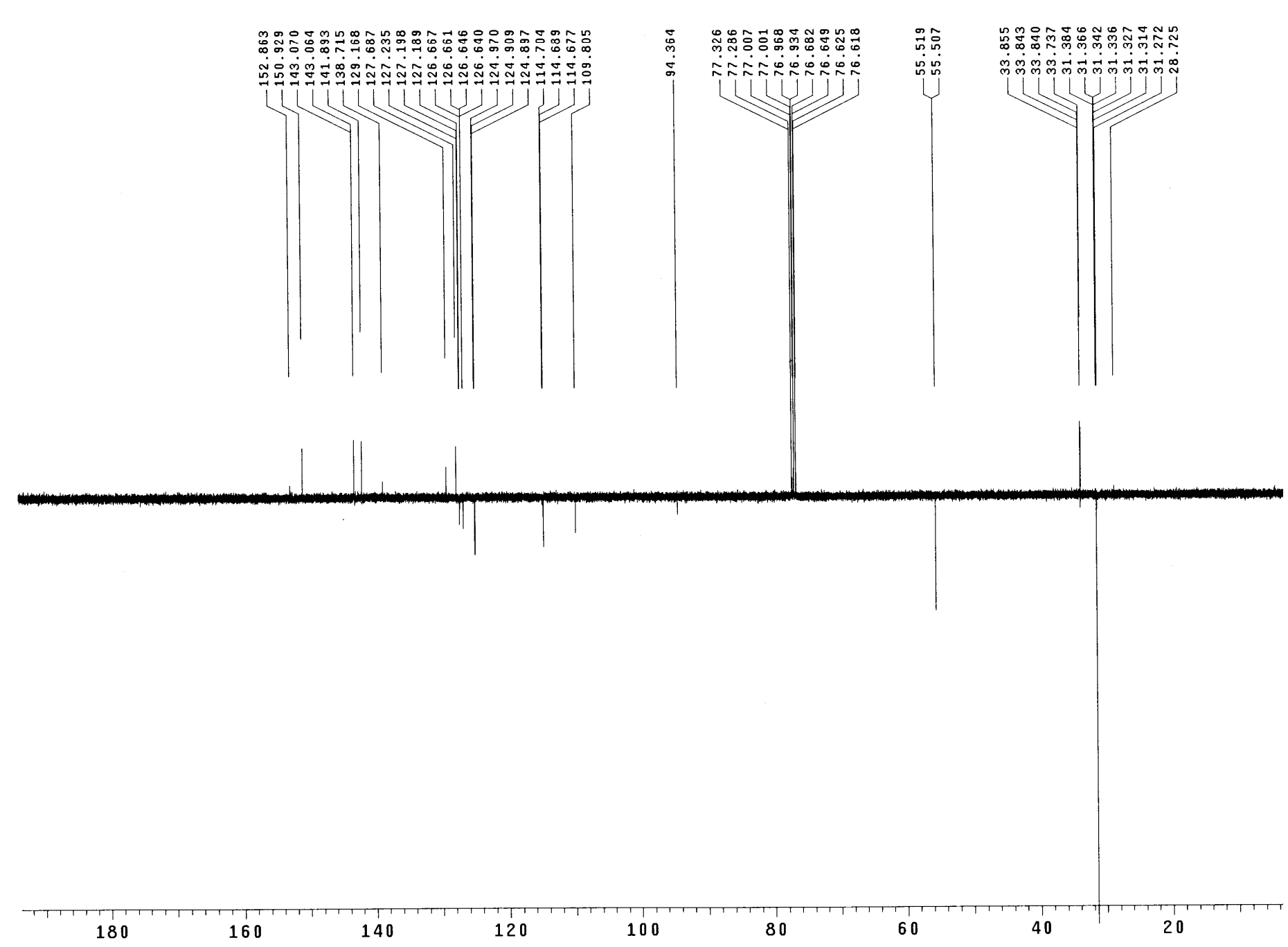

Pulse Sequence: apt

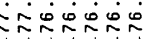

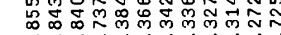

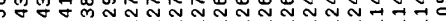


Appendix 2. (5-tert-Butyl-2-hydroxyphenyl)-tris(5-tert-butyl-2-methoxyphenyl)ethene 10. ${ }^{1} \mathrm{H}$ NMR spectrum.

$400 \mathrm{MHz}$ id in $\operatorname{COC} 13$ (ref. to $\operatorname{CDC} 13$ e $7.26 \mathrm{ppm}$ ), temp $26.8 \mathrm{C} \rightarrow$ actual temp $=27.0 \mathrm{C}$, asw400 probe

Pulse Sequence: s2pul

date:Feb $? 2007$ seqfil:s2pul hz:mm:16.fiz sweep width[Hz]:1002 spectrometer:i400 file:exp

(5-tert-butyl-2- hydroxyphenyl)-tris(5-tert-butyl-2-methoxyphenyl)ethene 10

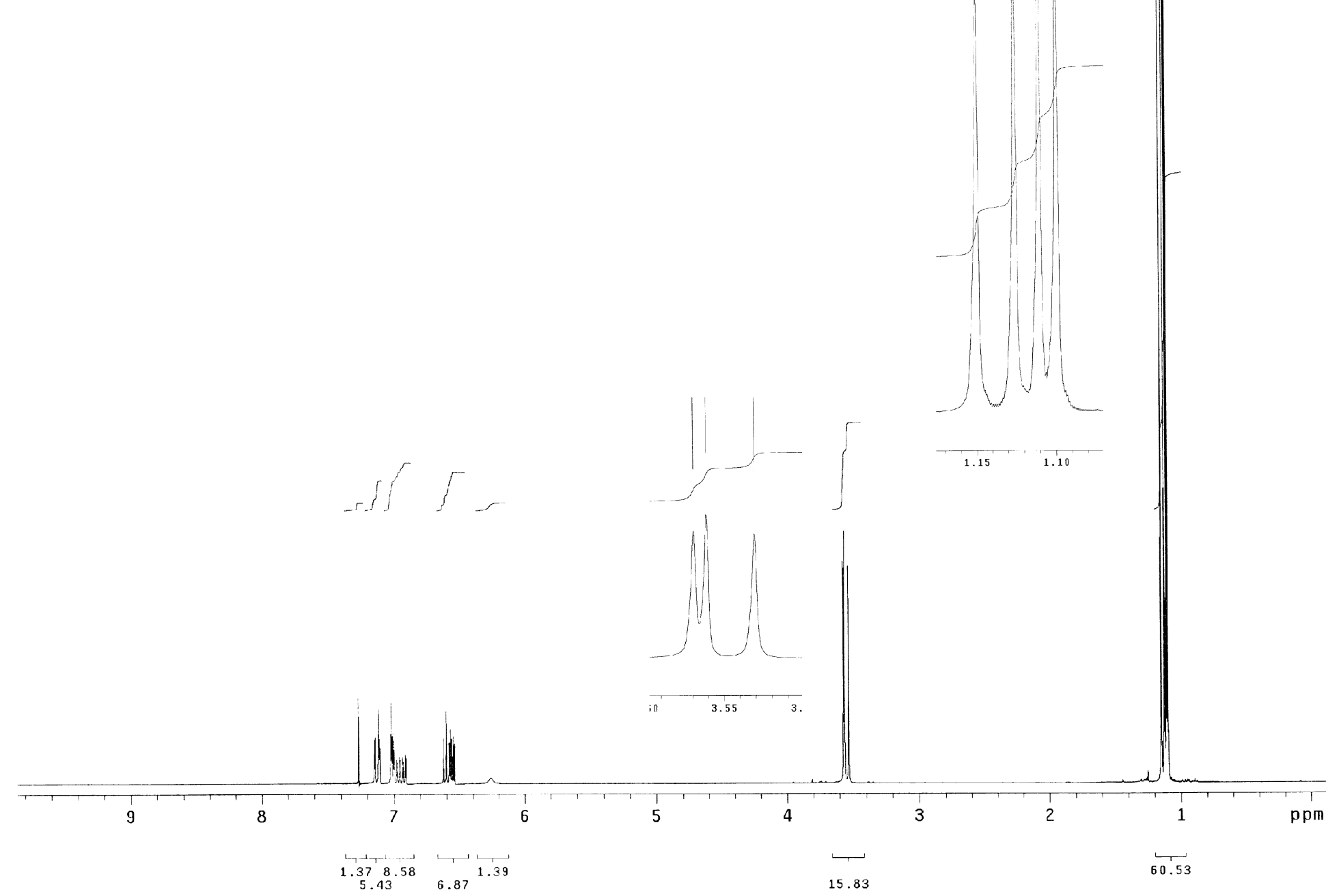


Appendix 2. (5-tert-Butyl-2-hydroxyphenyl)-tris(5-tert-butyl-2-methoxyphenyl)ethene 10. ${ }^{13} \mathrm{C}$-APT NMR spectrum.

$125 \mathrm{MHZ}$ APT in $\mathrm{CDCl} 13$ (ref. to $\mathrm{CDC} 13$ o $77.0 \mathrm{ppm})$, temp $27.2 \mathrm{C} \rightarrow$ actual temp $=27.0 \mathrm{C}$, sw probe
$\mathrm{C} \& \mathrm{CH}$ same, $\mathrm{CH} \& \mathrm{CH} 3$ opposite side of solvent'signal

Pulse Sequence: apt date:Feb 92004 seqfil:apt hz/mm:73.62 sweep width[Hz]:31299 spectrometer:ibd5 $\begin{array}{lll}\text { acq.time[s]:2.0 } & \text { relax. time[s]:0.1 } & \text { dig.res.[Hz/pt]:0.48 \# of scans:336 }\end{array}$

i 1 e: /mnt/d600/t11nmrdata/gzq/gzq-s-mono-oh-apt.fid

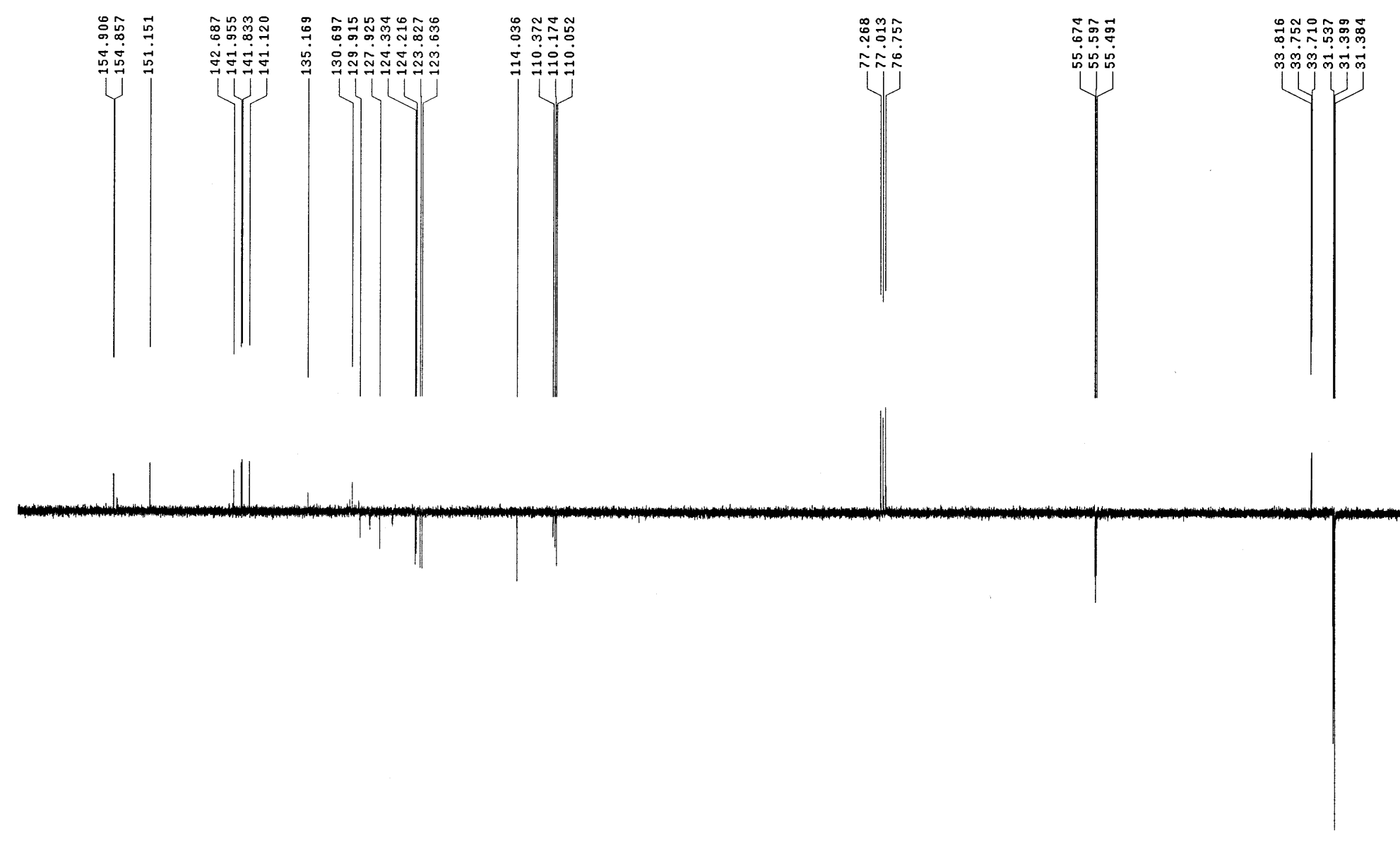

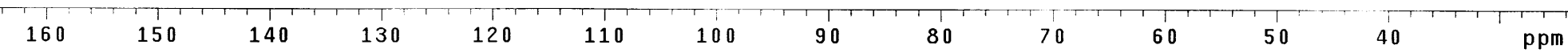




\section{X-ray Crystal Structure Report}

Compound: cis-1,2-Di(2-hydroxyphenyl)-1,2-di(2-methoxyphenyl)ethene $\mathbf{2 Z}$

Formula: $\quad \mathrm{C}_{28} \mathrm{H}_{24} \mathrm{O}_{4}$

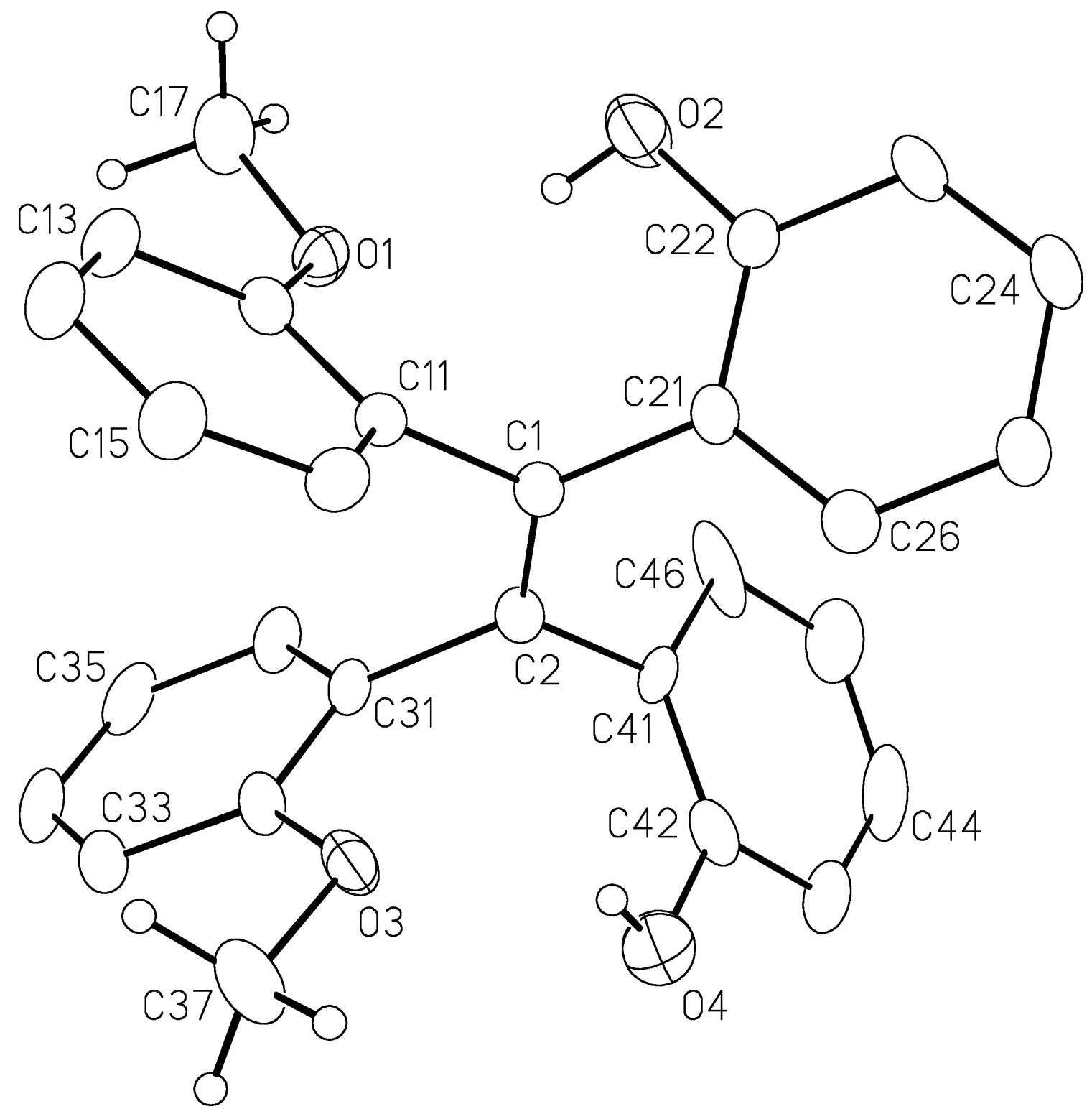

Figure 1. Perspective view of the cis-1,2-di(2-hydroxyphenyl)-1,2-di(2-methoxyphenyl)ethene molecule showing the atom labelling scheme. Non-hydrogen atoms are represented by Gaussian ellipsoids at the $20 \%$ probability level. Hydroxyl and methyl hydrogen atoms are shown with arbitrarily small thermal parameters; all other hydrogens are not shown. 


\section{List of Tables}

Table 1. Crystallographic Experimental Details

Table 2. Atomic Coordinates and Equivalent Isotropic Displacement Parameters

Table 3. Selected Interatomic Distances

Table 4. Selected Interatomic Angles

Table 5. Anisotropic Displacement Parameters

Table 7. Derived Atomic Coordinates and Displacement Parameters for Hydrogen Atoms

Table 1. Crystallographic Experimental Details
A. Crystal Data
formula
$\mathrm{C}_{28} \mathrm{H}_{24} \mathrm{O}_{4}$
formula weight
424.47
crystal dimensions ( $\mathrm{mm}$ )
$0.25 \times 0.12 \times 0.04$
crystal system
monoclinic
space group
$P 2{ }_{1} / c$ (No. 14)
unit cell parameters ${ }^{a}$
$a(\AA)$
$10.9757(13)$
$b(\AA)$
$15.4466(18)$
$c(\AA)$
$13.6421(13)$
$\beta(\operatorname{deg})$
$99.547(2)$
$V\left(\AA^{3}\right)$
$2280.8(4)$
$Z$
4
$\rho_{\text {calcd }}\left(\mathrm{g} \mathrm{cm}^{-3}\right)$
1.236
$\mu\left(\mathrm{mm}^{-1}\right)$
0.082

B. Data Collection and Refinement Conditions

diffractometer radiation $(\lambda[\AA])$

temperature $\left({ }^{\circ} \mathrm{C}\right)$

scan type

data collection $2 \theta$ limit (deg)

total data collected

independent reflections

number of observations $(N O)$

structure solution method

refinement method
Bruker P4/RA/SMART $1000 \mathrm{CCD}^{b}$

graphite-monochromated Mo $\mathrm{K} \alpha(0.71073)$

$-80$

$\phi$ rotations $\left(0.3^{\circ}\right) / \omega$ scans $\left(0.3^{\circ}\right)(30 \mathrm{~s}$ exposures $)$

51.60

$12189(-13 \leq h \leq 13,-18 \leq k \leq 18,-16 \leq l \leq 15)$

$4356\left(R_{\text {int }}=0.1625\right)$

$1167\left[F_{\mathrm{o}}^{2} \geq 2 \sigma\left(F_{\mathrm{o}}^{2}\right)\right]$

direct methods (SHELXS-86 $)$

full-matrix least-squares on $F^{2}\left(S H E L X L-93^{d}\right)$ 
absorption correction method

range of transmission factors

data/restraints/parameters

extinction coefficient $(x)^{e}$

goodness-of-fit $(S)^{f}$

final $R$ indices $g$

$$
\begin{aligned}
& R_{1}\left[F_{\mathrm{O}}^{2} \geq 2 \sigma\left(F_{\mathrm{O}}^{2}\right)\right] \\
& w R_{2}\left[F_{\mathrm{O}}^{2} \geq-3 \sigma\left(F_{\mathrm{O}}^{2}\right)\right]
\end{aligned}
$$

largest difference peak and hole multi-scan (SADABS)

0.9674-0.6240

$4356\left[F_{\mathrm{o}}^{2} \geq-3 \sigma\left(F_{\mathrm{o}}^{2}\right)\right] / 0 / 292$

$0.0116(13)$

$0.771\left[F_{\mathrm{o}}^{2} \geq-3 \sigma\left(F_{\mathrm{o}}^{2}\right)\right]$

0.0659

0.1837

0.631 and -0.276 e $\AA^{-3}$

${ }^{a}$ Obtained from least-squares refinement of 1526 centered reflections.

$b$ Programs for diffractometer operation, data collection, data reduction and absorption correction were those supplied by Bruker.

cSheldrick, G. M. Acta Crystallogr. 1990, A46, 467-473.

${ }^{d}$ Sheldrick, G. M. SHELXL-93. Program for crystal structure determination. University of Göttingen, Germany, 1993. Refinement on $F_{\mathrm{O}}{ }^{2}$ for all reflections (all of these having $F_{\mathrm{O}}{ }^{2} \geq$ $-3 \sigma\left(F_{\mathrm{o}}^{2}\right)$ ). Weighted $R$-factors $w R_{2}$ and all goodnesses of fit $S$ are based on $F_{\mathrm{o}}{ }^{2}$; conventional $R$-factors $R_{1}$ are based on $F_{\mathrm{o}}$, with $F_{\mathrm{o}}$ set to zero for negative $F_{\mathrm{O}}{ }^{2}$. The observed criterion of $F_{\mathrm{O}}^{2}>2 \sigma\left(F_{\mathrm{O}}{ }^{2}\right)$ is used only for calculating $R_{1}$, and is not relevant to the choice of reflections for refinement. $R$-factors based on $F_{\mathrm{O}}{ }^{2}$ are statistically about twice as large as those based on $F_{\mathrm{O}}$, and $R$-factors based on ALL data will be even larger.

$e F_{\mathrm{c}} *=k F_{\mathrm{c}}\left[1+x\left\{0.001 F_{\mathrm{c}}{ }^{2} \lambda^{3} / \sin (2 \theta)\right\}\right]^{-1 / 4}$ where $k$ is the overall scale factor.

$f_{S}=\left[\Sigma w\left(F_{\mathrm{o}}{ }^{2}-F_{\mathrm{c}^{2}}\right)^{2} /(n-p)\right]^{1 / 2}(n=$ number of data; $p=$ number of parameters varied; $w=$ $\left[\sigma^{2}\left(F_{\mathrm{o}}{ }^{2}\right)+(0.0622 P)^{2}\right]^{-1}$ where $\left.P=\left[\operatorname{Max}\left(F_{\mathrm{o}}^{2}, 0\right)+2 F_{\mathrm{c}}^{2}\right] / 3\right)$.

$g R_{1}=\Sigma|| F_{\mathrm{o}}|-| F_{\mathrm{c}}|| / \Sigma\left|F_{\mathrm{o}}\right| ; w R_{2}=\left[\Sigma w\left(F_{\mathrm{o}}{ }^{2}-F_{\mathrm{c}}{ }^{2}\right)^{2} / \Sigma w\left(F_{\mathrm{o}}{ }^{4}\right)\right]^{1 / 2}$. 
Table 2. Atomic Coordinates and Equivalent Isotropic Displacement Parameters

\begin{tabular}{lrlll} 
Atom & \multicolumn{1}{l}{$y$} & $y$ & $z$ & $U_{\text {eq }} \AA^{2}$ \\
O1 & $0.4055(3)$ & $-0.3307(2)$ & $0.5354(2)$ & $0.0558(10)^{*}$ \\
O2 & $0.3979(4)$ & $-0.1525(2)$ & $0.5096(3)$ & $0.0894(14)^{*}$ \\
O3 & $0.1331(3)$ & $-0.4187(2)$ & $0.2159(3)$ & $0.0636(11)^{*}$ \\
O4 & $-0.0828(4)$ & $-0.3190(3)$ & $0.2451(4)$ & $0.1117(16)^{*}$ \\
C1 & $0.2437(5)$ & $-0.2692(3)$ & $0.3724(3)$ & $0.0399(13)^{*}$ \\
C2 & $0.1420(5)$ & $-0.3140(3)$ & $0.3836(3)$ & $0.0418(13)^{*}$ \\
C11 & $0.3600(4)$ & $-0.3163(3)$ & $0.3630(4)$ & $0.0404(13)^{*}$ \\
C12 & $0.4381(5)$ & $-0.3501(3)$ & $0.4432(4)$ & $0.0454(13)^{*}$ \\
C13 & $0.5421(5)$ & $-0.3962(3)$ & $0.4342(4)$ & $0.0599(16)^{*}$ \\
C14 & $0.5685(5)$ & $-0.4114(4)$ & $0.3389(4)$ & $0.0637(16)^{*}$ \\
C15 & $0.4933(5)$ & $-0.3770(3)$ & $0.2568(4)$ & $0.0610(16)^{*}$ \\
C16 & $0.3895(5)$ & $-0.3307(3)$ & $0.2701(4)$ & $0.0507(14)^{*}$ \\
C17 & $0.4892(5)$ & $-0.3560(4)$ & $0.6235(4)$ & $0.083(2)^{*}$ \\
C21 & $0.2445(5)$ & $-0.1726(3)$ & $0.3628(4)$ & $0.0412(13)^{*}$ \\
C22 & $0.3219(5)$ & $-0.1205(4)$ & $0.4292(4)$ & $0.0512(14)^{*}$ \\
C23 & $0.3226(5)$ & $-0.0317(4)$ & $0.4179(4)$ & $0.0616(16)^{*}$ \\
C24 & $0.2472(5)$ & $0.0072(3)$ & $0.3401(4)$ & $0.0554(15)^{*}$ \\
C25 & $0.1704(5)$ & $-0.0423(4)$ & $0.2723(4)$ & $0.0539(15)^{*}$ \\
C26 & $0.1687(4)$ & $-0.1318(4)$ & $0.2840(4)$ & $0.0505(14)^{*}$ \\
C31 & $0.1442(4)$ & $-0.4117(3)$ & $0.3881(5)$ & $0.0488(14)^{*}$ \\
C32 & $0.1436(5)$ & $-0.4632(4)$ & $0.3046(5)$ & $0.0565(15)^{*}$ \\
C33 & $0.1506(5)$ & $-0.5522(4)$ & $0.3118(5)$ & $0.0648(17)^{*}$ \\
C34 & $0.1545(5)$ & $-0.5912(4)$ & $0.4020(6)$ & $0.077(2)^{*}$ \\
C35 & $0.1512(5)$ & $-0.5427(4)$ & $0.4864(5)$ & $0.0691(18)^{*}$ \\
C36 & $0.1471(4)$ & $-0.4532(4)$ & $0.4797(4)$ & $0.0596(16)^{*}$ \\
C37 & $0.1461(6)$ & $-0.4685(4)$ & $0.1304(4)$ & $0.089(2)^{*}$ \\
C41 & $0.0236(4)$ & $-0.2714(3)$ & $0.4013(4)$ & $0.0495(15)^{*}$ \\
C42 & $-0.0800(6)$ & $-0.2780(3)$ & $0.3311(5)$ & $0.0599(16)^{*}$ \\
C43 & $-0.1949(5)$ & $-0.2457(4)$ & $0.3408(6)$ & $0.0742(19)^{*}$ \\
C44 & $-0.2002(7)$ & $-0.2007(4)$ & $0.4267(6)$ & $0.090(2)^{*}$ \\
C45 & $-0.0962(7)$ & $-0.1918(4)$ & $0.4976(5)$ & $0.084(2)^{*}$ \\
C46 & $0.0218(7)$ & $-0.2269(3)$ & $0.4912(4)$ & $0.080(2)^{*}$
\end{tabular}

Anisotropically-refined atoms are marked with an asterisk (*). The form of the anisotropic displacement parameter is: $\exp \left[-2 \pi^{2}\left(h^{2} a^{* 2} U_{11}+k^{2} b^{* 2} U_{22}+l^{2} c^{* 2} U_{33}+2 k l b * c^{*} U_{23}\right.\right.$ $\left.\left.+2 h l a^{*} c^{*} U_{13}+2 h k a^{*} b^{*} U_{12}\right)\right]$. 
Table 3. Selected Interatomic Distances ( $\AA$ )

\begin{tabular}{|c|c|c|c|c|c|}
\hline Atom1 & Atom2 & Distance & Atom1 & Atom2 & Distance \\
\hline $\mathrm{O} 1$ & C12 & $1.395(5)$ & C21 & C26 & $1.396(6)$ \\
\hline $\mathrm{O} 1$ & $\mathrm{C} 17$ & $1.441(5)$ & $\mathrm{C} 22$ & $\mathrm{C} 23$ & $1.381(6)$ \\
\hline $\mathrm{O} 2$ & $\mathrm{C} 22$ & $1.356(5)$ & $\mathrm{C} 23$ & $\mathrm{C} 24$ & $1.371(6)$ \\
\hline $\mathrm{O} 3$ & $\mathrm{C} 32$ & $1.380(6)$ & $\mathrm{C} 24$ & $\mathrm{C} 25$ & $1.376(6)$ \\
\hline $\mathrm{O} 3$ & C37 & $1.424(5)$ & $\mathrm{C} 25$ & $\mathrm{C} 26$ & $1.393(6)$ \\
\hline $\mathrm{O} 4$ & $\mathrm{C} 42$ & $1.329(6)$ & $\mathrm{C} 31$ & $\mathrm{C} 32$ & $1.388(6)$ \\
\hline $\mathrm{C} 1$ & $\mathrm{C} 2$ & $1.344(6)$ & $\mathrm{C} 31$ & $\mathrm{C} 36$ & $1.399(6)$ \\
\hline $\mathrm{C} 1$ & $\mathrm{C} 11$ & $1.493(6)$ & $\mathrm{C} 32$ & $\mathrm{C} 33$ & $1.379(7)$ \\
\hline $\mathrm{C} 1$ & $\mathrm{C} 21$ & $1.497(6)$ & C33 & C34 & $1.364(7)$ \\
\hline $\mathrm{C} 2$ & $\mathrm{C} 31$ & $1.511(6)$ & C34 & C35 & $1.379(7)$ \\
\hline $\mathrm{C} 2$ & $\mathrm{C} 41$ & $1.511(6)$ & $\mathrm{C} 35$ & $\mathrm{C} 36$ & $1.387(7)$ \\
\hline C11 & $\mathrm{C} 12$ & $1.376(6)$ & $\mathrm{C} 41$ & $\mathrm{C} 42$ & $1.363(7)$ \\
\hline C11 & $\mathrm{C} 16$ & $1.378(6)$ & $\mathrm{C} 41$ & $\mathrm{C} 46$ & $1.409(7)$ \\
\hline $\mathrm{C} 12$ & $\mathrm{C} 13$ & $1.368(6)$ & $\mathrm{C} 42$ & $\mathrm{C} 43$ & $1.382(7)$ \\
\hline $\mathrm{C} 13$ & $\mathrm{C} 14$ & $1.398(6)$ & $\mathrm{C} 43$ & C44 & $1.372(8)$ \\
\hline $\mathrm{C} 14$ & $\mathrm{C} 15$ & $1.384(6)$ & C44 & $\mathrm{C} 45$ & $1.375(8)$ \\
\hline $\mathrm{C} 15$ & $\mathrm{C} 16$ & $1.382(6)$ & $\mathrm{C} 45$ & C46 & $1.421(7)$ \\
\hline $\mathrm{C} 21$ & $\mathrm{C} 22$ & $1.391(6)$ & & & \\
\hline
\end{tabular}


Table 4. Selected Interatomic Angles (deg)

$\begin{array}{llllllll}\text { Atom1 } & \text { Atom2 } & \text { Atom3 } & \text { Angle } & \text { Atom1 } & \text { Atom2 } & \text { Atom3 } & \text { Angle } \\ \text { C12 } & \text { O1 } & \text { C17 } & 118.0(4) & \text { C22 } & \text { C23 } & \text { C24 } & 120.6(5) \\ \text { C32 } & \text { O3 } & \text { C37 } & 116.4(4) & \text { C23 } & \text { C24 } & \text { C25 } & 120.1(5) \\ \text { C2 } & \text { C1 } & \text { C11 } & 119.8(4) & \text { C24 } & \text { C25 } & \text { C26 } & 119.5(5) \\ \text { C2 } & \text { C1 } & \text { C21 } & 122.7(5) & \text { C21 } & \text { C26 } & \text { C25 } & 121.3(5) \\ \text { C11 } & \text { C1 } & \text { C21 } & 117.4(4) & \text { C2 } & \text { C31 } & \text { C32 } & 122.7(5) \\ \text { C1 } & \text { C2 } & \text { C31 } & 120.7(5) & \text { C2 } & \text { C31 } & \text { C36 } & 119.4(5) \\ \text { C1 } & \text { C2 } & \text { C41 } & 123.2(4) & \text { C32 } & \text { C31 } & \text { C36 } & 117.8(5) \\ \text { C31 } & \text { C2 } & \text { C41 } & 115.9(4) & \text { O3 } & \text { C32 } & \text { C31 } & 115.0(5) \\ \text { C1 } & \text { C11 } & \text { C12 } & 123.1(4) & \text { O3 } & \text { C32 } & \text { C33 } & 123.7(6) \\ \text { C1 } & \text { C11 } & \text { C16 } & 119.5(4) & \text { C31 } & \text { C32 } & \text { C33 } & 121.3(6) \\ \text { C12 } & \text { C11 } & \text { C16 } & 117.3(5) & \text { C32 } & \text { C33 } & \text { C34 } & 119.8(6) \\ \text { O1 } & \text { C12 } & \text { C11 } & 114.5(5) & \text { C33 } & \text { C34 } & \text { C35 } & 120.8(6) \\ \text { O1 } & \text { C12 } & \text { C13 } & 122.4(5) & \text { C34 } & \text { C35 } & \text { C36 } & 119.5(6) \\ \text { C11 } & \text { C12 } & \text { C13 } & 123.0(5) & \text { C31 } & \text { C36 } & \text { C35 } & 120.7(6) \\ \text { C12 } & \text { C13 } & \text { C14 } & 118.4(5) & \text { C2 } & \text { C41 } & \text { C42 } & 119.7(5) \\ \text { C13 } & \text { C14 } & \text { C15 } & 120.1(5) & \text { C2 } & \text { C41 } & \text { C46 } & 119.2(5) \\ \text { C14 } & \text { C15 } & \text { C16 } & 119.1(5) & \text { C42 } & \text { C41 } & \text { C46 } & 121.1(5) \\ \text { C11 } & \text { C16 } & \text { C15 } & 122.0(5) & \text { O4 } & \text { C42 } & \text { C41 } & 123.3(6) \\ \text { C1 } & \text { C21 } & \text { C22 } & 122.1(5) & \text { O4 } & \text { C42 } & \text { C43 } & 111.7(7) \\ \text { C1 } & \text { C21 } & \text { C26 } & 120.3(5) & \text { C41 } & \text { C42 } & \text { C43 } & 125.0(6) \\ \text { C22 } & \text { C21 } & \text { C26 } & 117.5(5) & \text { C42 } & \text { C43 } & \text { C44 } & 115.7(6) \\ \text { O2 } & \text { C22 } & \text { C21 } & 122.9(5) & \text { C43 } & \text { C44 } & \text { C45 } & 120.2(7) \\ \text { O2 } & \text { C22 } & \text { C23 } & 116.0(5) & \text { C44 } & \text { C45 } & \text { C46 } & 125.3(7) \\ \text { C21 } & \text { C22 } & \text { C23 } & 121.1(5) & \text { C41 } & \text { C46 } & \text { C45 } & 112.6(6)\end{array}$


Table 5. Anisotropic Displacement Parameters $\left(U_{\mathrm{ij}}, \AA^{2}\right)$

\begin{tabular}{|c|c|c|c|c|c|c|}
\hline Atom & $U_{11}$ & $U_{22}$ & $U_{33}$ & $U_{23}$ & $U_{13}$ & $U_{12}$ \\
\hline $\mathrm{O} 1$ & $0.059(2)$ & $0.056(2)$ & $0.049(2)$ & $0.002(2)$ & $0.0004(19)$ & $0.008(2)$ \\
\hline $\mathrm{O} 2$ & $0.131(4)$ & $0.051(3)$ & $0.072(3)$ & $0.002(2)$ & $-0.022(3)$ & $-0.004(3)$ \\
\hline $\mathrm{O} 3$ & $0.086(3)$ & $0.037(2)$ & $0.064(2)$ & $-0.010(2)$ & $0.002(2)$ & $0.002(2)$ \\
\hline $\mathrm{O} 4$ & $0.086(3)$ & $0.083(4)$ & $0.155(4)$ & $-0.010(4)$ & $-0.012(3)$ & $0.003(3)$ \\
\hline $\mathrm{C} 1$ & $0.043(3)$ & $0.036(3)$ & $0.041(3)$ & $0.004(3)$ & $0.008(3)$ & $0.000(3)$ \\
\hline $\mathrm{C} 2$ & $0.047(3)$ & $0.037(3)$ & $0.042(3)$ & $0.005(3)$ & $0.010(3)$ & $0.001(3)$ \\
\hline $\mathrm{C} 11$ & $0.040(3)$ & $0.035(3)$ & $0.046(3)$ & $0.003(3)$ & $0.006(3)$ & $-0.002(3)$ \\
\hline C12 & $0.047(3)$ & $0.042(4)$ & $0.050(3)$ & $-0.006(3)$ & $0.015(3)$ & $0.000(3)$ \\
\hline C13 & $0.049(4)$ & $0.062(4)$ & $0.067(4)$ & $0.005(3)$ & $0.006(3)$ & $0.015(3)$ \\
\hline $\mathrm{C} 14$ & $0.050(4)$ & $0.064(4)$ & $0.078(4)$ & $0.002(4)$ & $0.016(3)$ & $0.018(3)$ \\
\hline $\mathrm{C} 15$ & $0.052(3)$ & $0.064(4)$ & $0.071(4)$ & $0.006(4)$ & $0.023(3)$ & $0.006(3)$ \\
\hline $\mathrm{C} 16$ & $0.045(3)$ & $0.054(4)$ & $0.054(4)$ & $0.008(3)$ & $0.009(3)$ & $-0.001(3)$ \\
\hline $\mathrm{C} 17$ & $0.115(5)$ & $0.071(5)$ & $0.060(4)$ & $0.003(3)$ & $0.001(4)$ & $0.025(4)$ \\
\hline $\mathrm{C} 21$ & $0.046(3)$ & $0.035(3)$ & $0.045(3)$ & $0.002(3)$ & $0.014(3)$ & $0.005(3)$ \\
\hline $\mathrm{C} 22$ & $0.059(4)$ & $0.043(4)$ & $0.047(3)$ & $0.004(3)$ & $-0.006(3)$ & $0.006(3)$ \\
\hline $\mathrm{C} 23$ & $0.083(5)$ & $0.031(4)$ & $0.069(4)$ & $-0.003(3)$ & $0.006(3)$ & $-0.007(3)$ \\
\hline $\mathrm{C} 24$ & $0.061(4)$ & $0.032(3)$ & $0.079(4)$ & $0.009(4)$ & $0.028(3)$ & $0.000(3)$ \\
\hline $\mathrm{C} 25$ & $0.052(4)$ & $0.041(4)$ & $0.070(4)$ & $0.013(3)$ & $0.016(3)$ & $0.001(3)$ \\
\hline $\mathrm{C} 26$ & $0.047(3)$ & $0.047(4)$ & $0.059(4)$ & $0.000(3)$ & $0.015(3)$ & $0.000(3)$ \\
\hline C31 & $0.032(3)$ & $0.030(3)$ & $0.083(4)$ & $0.012(4)$ & $0.004(3)$ & $0.001(3)$ \\
\hline C32 & $0.052(4)$ & $0.026(4)$ & $0.090(5)$ & $0.009(4)$ & $0.006(3)$ & $-0.002(3)$ \\
\hline $\mathrm{C} 33$ & $0.055(4)$ & $0.035(4)$ & $0.100(5)$ & $-0.001(4)$ & $0.001(4)$ & $0.004(3)$ \\
\hline C34 & $0.046(4)$ & $0.039(4)$ & $0.144(6)$ & $0.022(5)$ & $0.011(4)$ & $0.012(3)$ \\
\hline C35 & $0.042(4)$ & $0.062(5)$ & $0.105(5)$ & $0.041(4)$ & $0.015(4)$ & $0.012(3)$ \\
\hline $\mathrm{C} 36$ & $0.048(4)$ & $0.044(4)$ & $0.088(4)$ & $0.023(4)$ & $0.014(3)$ & $0.007(3)$ \\
\hline C37 & $0.127(6)$ & $0.050(4)$ & $0.090(4)$ & $-0.015(4)$ & $0.014(4)$ & $0.003(4)$ \\
\hline $\mathrm{C} 41$ & $0.027(3)$ & $0.032(3)$ & $0.086(4)$ & $0.028(3)$ & $0.002(3)$ & $0.000(3)$ \\
\hline $\mathrm{C} 42$ & $0.075(5)$ & $0.032(4)$ & $0.076(4)$ & $-0.005(3)$ & $0.021(4)$ & $0.001(4)$ \\
\hline $\mathrm{C} 43$ & $0.039(4)$ & $0.049(4)$ & $0.134(6)$ & $0.033(4)$ & $0.012(4)$ & $0.004(3)$ \\
\hline $\mathrm{C} 44$ & $0.084(6)$ & $0.058(5)$ & $0.144(7)$ & $0.050(5)$ & $0.063(6)$ & $0.029(4)$ \\
\hline $\mathrm{C} 45$ & $0.100(6)$ & $0.076(5)$ & $0.085(5)$ & $0.026(4)$ & $0.040(5)$ & $0.021(5)$ \\
\hline $\mathrm{C} 46$ & $0.133(6)$ & $0.032(4)$ & $0.104(5)$ & $-0.001(4)$ & $0.097(5)$ & $0.011(4)$ \\
\hline
\end{tabular}

The form of the anisotropic displacement parameter is:

$\exp \left[-2 \pi^{2}\left(h^{2} a^{* 2} U_{11}+k^{2} b^{* 2} U_{22}+l^{2} c^{* 2} U_{33}+2 k l b^{*} c^{*} U_{23}+2 h l a^{*} c^{*} U_{13}+2 h k a^{*} b^{*} U_{12}\right)\right]$ 
Table 6. Derived Atomic Coordinates and Displacement Parameters for Hydrogen Atoms

$\begin{array}{lcccc}\text { Atom } & x & y & z & U_{\mathrm{eq}}, \AA^{2} \\ \text { H2O } & 0.3791 & -0.2042 & 0.5187 & 0.134 \\ \mathrm{H} 4 \mathrm{O} & -0.0107 & -0.3230 & 0.2324 & 0.168 \\ \mathrm{H} 13 & 0.5951 & -0.4174 & 0.4913 & 0.072 \\ \mathrm{H} 14 & 0.6381 & -0.4455 & 0.3306 & 0.076 \\ \mathrm{H} 15 & 0.5127 & -0.3850 & 0.1920 & 0.073 \\ \mathrm{H} 16 & 0.3370 & -0.3082 & 0.2135 & 0.061 \\ \mathrm{H} 17 \mathrm{~A} & 0.4547 & -0.3392 & 0.6825 & 0.100 \\ \mathrm{H} 17 \mathrm{~B} & 0.5689 & -0.3271 & 0.6246 & 0.100 \\ \mathrm{H} 17 \mathrm{C} & 0.5011 & -0.4189 & 0.6233 & 0.100 \\ \mathrm{H} 23 & 0.3757 & 0.0028 & 0.4643 & 0.074 \\ \mathrm{H} 24 & 0.2480 & 0.0684 & 0.3331 & 0.066 \\ \mathrm{H} 25 & 0.1189 & -0.0156 & 0.2178 & 0.065 \\ \mathrm{H} 26 & 0.1149 & -0.1658 & 0.2375 & 0.061 \\ \mathrm{H} 33 & 0.1526 & -0.5862 & 0.2541 & 0.078 \\ \mathrm{H} 34 & 0.1594 & -0.6525 & 0.4067 & 0.092 \\ \mathrm{H} 35 & 0.1518 & -0.5705 & 0.5486 & 0.083 \\ \mathrm{H} 36 & 0.1462 & -0.4196 & 0.5379 & 0.071 \\ \mathrm{H} 37 \mathrm{~A} & 0.1367 & -0.4306 & 0.0720 & 0.107 \\ \mathrm{H} 37 \mathrm{~B} & 0.0824 & -0.5136 & 0.1204 & 0.107 \\ \mathrm{H} 37 \mathrm{C} & 0.2280 & -0.4954 & 0.1401 & 0.107 \\ \mathrm{H} 43 & -0.2656 & -0.2541 & 0.2912 & 0.089 \\ \mathrm{H} 44 & -0.2760 & -0.1757 & 0.4373 & 0.108 \\ \mathrm{H} 45 & -0.1040 & -0.1595 & 0.5555 & 0.101 \\ \mathrm{H} 46 & 0.0919 & -0.2210 & 0.5419 & 0.097\end{array}$




\section{X-ray Crystal Structure Report}

\section{Cis-4b,9b-dihydro-4b,9b-bis(2-hydroxyphenyl)benzofuro(3,2-b)benzofuran 8}

Compound: $\quad\left\{\mathrm{C}\left(\mathrm{C}_{6} \mathrm{H}_{4}-2-\mathrm{OH}\right)\left(\mathrm{C}_{6} \mathrm{H}_{4}-2-\mathrm{O}-\right)\right\}_{2} \cdot \mathrm{Et}_{2} \mathrm{O}$

Formula: $\quad \mathrm{C}_{30} \mathrm{H}_{28} \mathrm{O}_{5}\left(\mathrm{C}_{26} \mathrm{H}_{18} \mathrm{O}_{4} \cdot \mathrm{C}_{4} \mathrm{H}_{10} \mathrm{O}\right)$

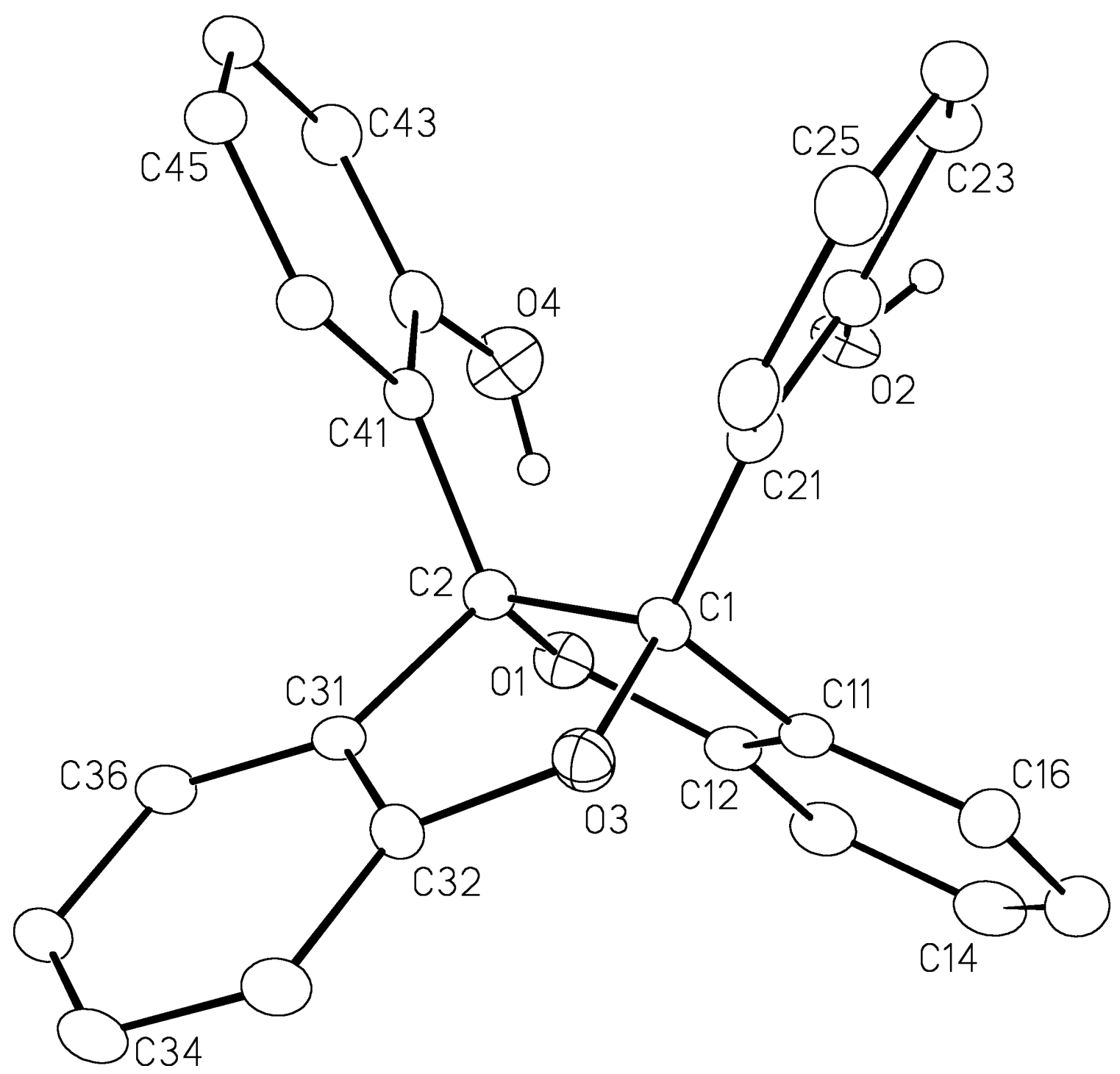

Figure 1. Perspective view of the $\left\{\mathrm{C}\left(\mathrm{C}_{6} \mathrm{H}_{4}-2-\mathrm{OH}\right)\left(\mathrm{C}_{6} \mathrm{H}_{4}-2-\mathrm{O}-\right)\right\}_{2}$ molecule showing the atom labelling scheme. Non-hydrogen atoms are represented by Gaussian ellipsoids at the $20 \%$ probability level. Hydroxyl hydrogen atoms are shown with arbitrarily small thermal parameters; all other hydrogens are not shown. 


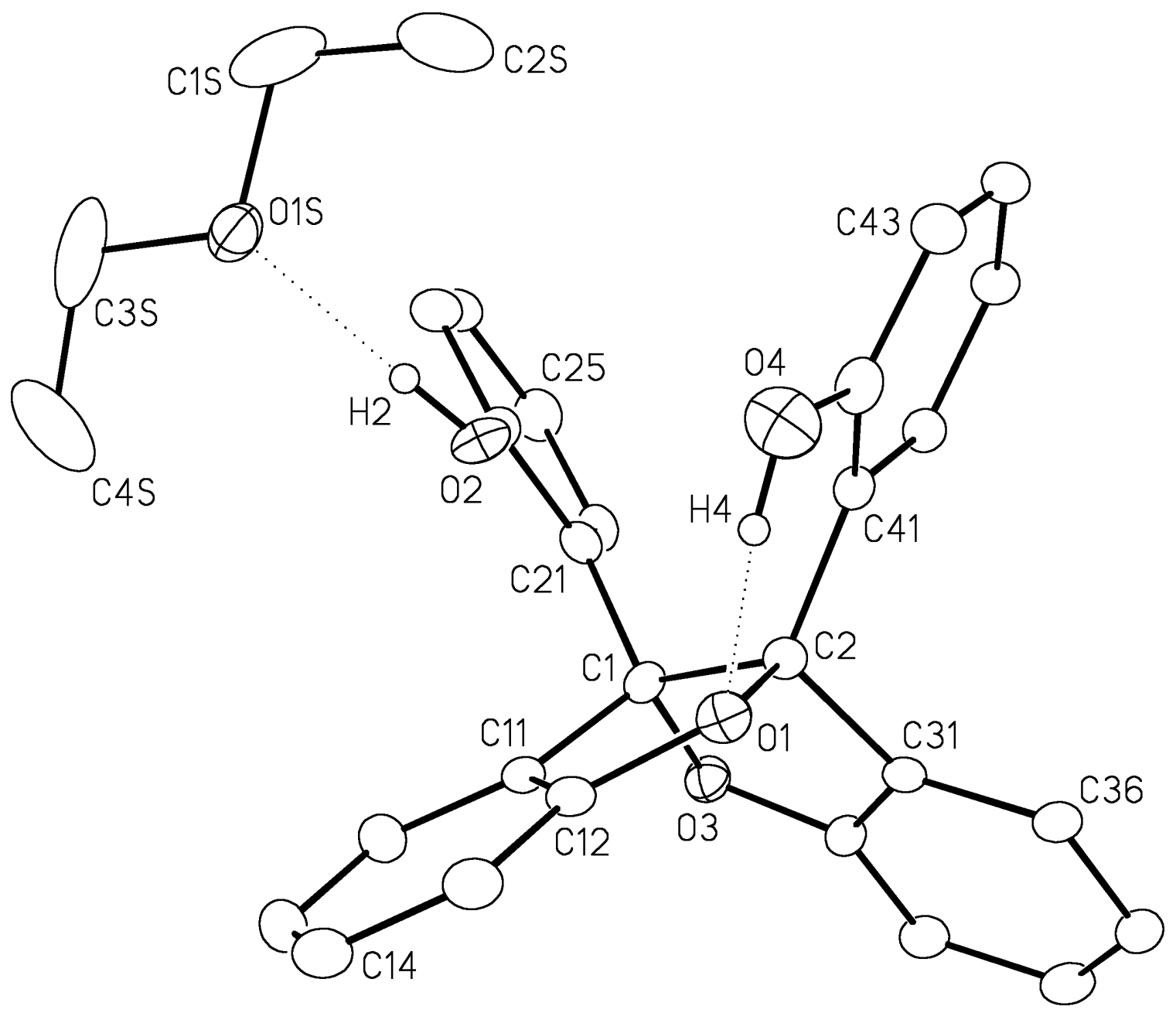

Figure 2. Alternate view showing the intra- $(\mathrm{O} 1 \cdots \mathrm{H} 4=1.86 \AA)$ and intermolecular $(\mathrm{O} 1 \mathrm{~S} \cdots \mathrm{H} 2=1.86 \AA)$ hydrogen-bonded interactions within and between the $\left\{\mathrm{C}\left(\mathrm{C}_{6} \mathrm{H}_{4}-2-\mathrm{OH}\right)\left(\mathrm{C}_{6} \mathrm{H}_{4}-2-\mathrm{O}-\right)\right\}_{2}$ molecule and the solvent diethyl ether molecule.

\section{List of Tables}

Table 1. Crystallographic Experimental Details

Table 2. Atomic Coordinates and Equivalent Isotropic Displacement Parameters

Table 3. Selected Interatomic Distances

Table 4. Selected Interatomic Angles

Table 5. Anisotropic Displacement Parameters

Table 6. Derived Atomic Coordinates and Displacement Parameters for Hydrogen Atoms 
Table 1. Crystallographic Experimental Details
A. Crystal Data
formula
$\mathrm{C}_{30} \mathrm{H}_{28} \mathrm{O}_{5}$
formula weight
468.52
crystal dimensions ( $\mathrm{mm}$ )
crystal system
triclinic
space group
$P$ I (No. 2)
unit cell parameters ${ }^{a}$

$\begin{array}{ll}a(\AA) & 8.8406(10) \\ b(\AA) & 9.5462(11) \\ c(\AA) & 16.604(2) \\ \alpha(\mathrm{deg}) & 88.872(2) \\ \beta(\mathrm{deg}) & 74.665(2) \\ \gamma(\mathrm{deg}) & 65.160(2) \\ V\left(\AA^{3}\right) & 1219.7(2) \\ Z & 2 \\ \rho_{\text {calcd }}\left(\mathrm{g} \mathrm{cm}^{-3}\right) & 1.276 \\ \left(\mathrm{~mm}^{-1}\right) & 0.086\end{array}$

$0.28 \times 0.08 \times 0.04$

B. Data Collection and Refinement Conditions

diffractometer

radiation $(\lambda[\AA]])$

temperature $\left({ }^{\circ} \mathrm{C}\right)$

scan type

data collection $2 \theta$ limit (deg)

total data collected

independent reflections

number of observations $(\mathrm{NO})$

structure solution method

refinement method

absorption correction method

data/restraints/parameters

goodness-of-fit $(S)^{f}$

final $R$ indices $g$

$$
\begin{array}{cl}
R_{1}\left[F_{\mathrm{O}}^{2} \geq 2 \sigma\left(F_{\mathrm{o}}^{2}\right)\right] & 0.0578 \\
w R_{2}\left[F_{\mathrm{o}}^{2} \geq-3 \sigma\left(F_{\mathrm{o}}^{2}\right)\right] & 0.1516 \\
\text { largest difference peak and hole } & 0.316 \text { and }-0.303 \mathrm{e} \AA^{-3}
\end{array}
$$

Bruker P4/RA/SMART $1000 \mathrm{CCD}^{b}$

graphite-monochromated Mo $\mathrm{K} \alpha(0.71073)$

$-80$

$\phi$ rotations $\left(0.3^{\circ}\right) / \omega$ scans $\left(0.3^{\circ}\right)$ (30 s exposures)

51.40

$6660(-9 \leq h \leq 10,-11 \leq k \leq 10,-20 \leq l \leq 19)$

$4614\left(R_{\text {int }}=0.0687\right)$

$1550\left[F_{\mathrm{O}}^{2} \geq 2 \sigma\left(F_{\mathrm{O}}^{2}\right)\right]$

direct methods (SHELXS-86 $)$

full-matrix least-squares on $F^{2}\left(S H E L X L-93^{d}\right)$

none $e^{e}$

$4614\left[F_{\mathrm{O}}^{2} \geq-3 \sigma\left(F_{\mathrm{O}}^{2}\right)\right] / 0 / 318$

$0.807\left[F_{\mathrm{O}}^{2} \geq-3 \sigma\left(F_{\mathrm{O}}^{2}\right)\right]$

${ }^{a}$ Obtained from least-squares refinement of 1532 centered reflections.

$b$ Programs for diffractometer operation, data collection, data reduction and absorption correction were those supplied by Bruker.

(continued) 
Table 1. Crystallographic Experimental Details (continued)

cSheldrick, G. M. Acta Crystallogr. 1990, A46, 467-473.

${ }^{d}$ Sheldrick, G. M. SHELXL-93. Program for crystal structure determination. University of Göttingen, Germany, 1993. Refinement on $F_{\mathrm{O}}{ }^{2}$ for all reflections (all of these having $F_{\mathrm{O}}{ }^{2} \geq$ $-3 \sigma\left(F_{\mathrm{o}}^{2}\right)$ ). Weighted $R$-factors $w R_{2}$ and all goodnesses of fit $S$ are based on $F_{\mathrm{o}}{ }^{2}$; conventional $R$-factors $R_{1}$ are based on $F_{\mathrm{o}}$, with $F_{\mathrm{O}}$ set to zero for negative $F_{\mathrm{O}}{ }^{2}$. The observed criterion of $F_{\mathrm{O}}^{2}>2 \sigma\left(F_{\mathrm{O}}{ }^{2}\right)$ is used only for calculating $R_{1}$, and is not relevant to the choice of reflections for refinement. $R$-factors based on $F_{\mathrm{O}}{ }^{2}$ are statistically about twice as large as those based on $F_{\mathrm{O}}$, and $R$-factors based on ALL data will be even larger.

${ }^{e}$ No absorption correction was applied due to the small crystal size and low value of the linear absorption coefficient $(\mu)$.

$f_{S}=\left[\Sigma w\left(F_{\mathrm{o}}^{2}-F_{\mathrm{c}^{2}}\right)^{2} /(n-p)\right]^{1 / 2}(n=$ number of data; $p=$ number of parameters varied; $w=$ $\left[\sigma^{2}\left(F_{\mathrm{o}}^{2}\right)+(0466 P)^{2}\right]^{-1}$ where $\left.P=\left[\operatorname{Max}\left(F_{\mathrm{o}}^{2}, 0\right)+2 F_{\mathrm{c}}^{2}\right] / 3\right)$.

$g R_{1}=\Sigma|| F_{\mathrm{o}}|-| F_{\mathrm{c}}|/ \Sigma| F_{\mathrm{o}} \mid ; w R_{2}=\left[\Sigma w\left(F_{\mathrm{o}}^{2}-F_{\mathrm{c}}^{2}\right)^{2 / \Sigma} w\left(F_{\mathrm{o}}{ }^{4}\right)\right]^{1 / 2}$. 
Table 2. Atomic Coordinates and Equivalent Isotropic Displacement Parameters

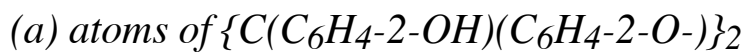

$\begin{array}{lllll}\text { Atom } & x & y & z & U_{\text {eq }}, \AA^{2} \\ \text { O1 } & 0.7918(3) & 0.4140(3) & 0.32005(17) & 0.0399(7)^{*} \\ \text { O2 } & 0.4160(3) & 0.6025(3) & 0.29920(18) & 0.0446(8)^{*} \\ \text { O3 } & 0.7960(3) & 0.1758(3) & 0.16455(17) & 0.0410(8)^{*} \\ \text { O4 } & 0.7040(5) & 0.7094(4) & 0.3249(2) & 0.0674(10)^{*} \\ \text { C1 } & 0.6852(5) & 0.3206(4) & 0.2198(3) & 0.0351(11)^{*} \\ \text { C2 } & 0.8118(5) & 0.3958(4) & 0.2299(2) & 0.0300(10)^{*} \\ \text { C11 } & 0.6270(5) & 0.2845(5) & 0.3084(3) & 0.0345(10)^{*} \\ \text { C12 } & 0.6936(5) & 0.3370(5) & 0.3604(3) & 0.0385(11)^{*} \\ \text { C13 } & 0.6595(6) & 0.3226(5) & 0.4452(3) & 0.0502(13)^{*} \\ \text { C14 } & 0.5582(6) & 0.2454(6) & 0.4765(3) & 0.0586(14)^{*} \\ \text { C15 } & 0.4904(6) & 0.1903(6) & 0.4249(3) & 0.0639(15)^{*} \\ \text { C16 } & 0.5232(5) & 0.2094(5) & 0.3405(3) & 0.0503(13)^{*} \\ \text { C21 } & 0.5371(5) & 0.4136(5) & 0.1823(3) & 0.0335(10)^{*} \\ \text { C22 } & 0.4024(5) & 0.5516(5) & 0.2257(3) & 0.0369(11)^{*} \\ \text { C23 } & 0.2627(5) & 0.6344(5) & 0.1949(3) & 0.0425(12)^{*} \\ \text { C24 } & 0.2560(5) & 0.5785(5) & 0.1204(3) & 0.0469(13)^{*} \\ \text { C25 } & 0.3895(6) & 0.4433(5) & 0.0755(3) & 0.0474(12)^{*} \\ \text { C26 } & 0.5293(5) & 0.3615(5) & 0.1069(3) & 0.0415(11)^{*} \\ \text { C31 } & 0.9864(5) & 0.2663(4) & 0.1877(2) & 0.0308(10)^{*} \\ \text { C32 } & 0.9656(5) & 0.1471(5) & 0.1545(3) & 0.0391(11)^{*} \\ \text { C33 } & 1.1041(5) & 0.0120(5) & 0.1110(3) & 0.0471(12)^{*} \\ \text { C34 } & 1.2684(6) & 0.0007(5) & 0.1037(3) & 0.0563(14)^{*} \\ \text { C35 } & 1.2944(5) & 0.1185(5) & 0.1370(3) & 0.0519(13)^{*} \\ \text { C36 } & 1.1521(5) & 0.2531(5) & 0.1792(3) & 0.0418(12)^{*} \\ \text { C41 } & 0.7864(4) & 0.5502(5) & 0.1942(3) & 0.0300(10)^{*} \\ \text { C42 } & 0.7442(5) & 0.6884(5) & 0.2397(3) & 0.0370(11)^{*} \\ \text { C43 } & 0.7356(5) & 0.8204(5) & 0.2007(3) & 0.0453(12)^{*} \\ \text { C44 } & 0.7666(5) & 0.8168(5) & 0.1155(3) & 0.0482(13)^{*} \\ \text { C45 } & 0.8057(5) & 0.6829(5) & 0.0675(3) & 0.0396(11)^{*} \\ \text { C46 } & 0.8178(5) & 0.5485(5) & 0.1061(3) & 0.0368(11)^{*} \\ & & & \end{array}$

(b) solvent diethyl ether atoms

$\begin{array}{lllll}\text { Atom } & x & y & z & U_{\text {eq }}, \AA^{2} \\ \text { O1S } & 0.1080(4) & 0.8266(4) & 0.38901(18) & 0.0547(9)^{*} \\ \text { C1S } & 0.0678(7) & 0.9846(6) & 0.3719(4) & 0.0840(19)^{*} \\ \text { C2S } & 0.2230(8) & 1.0116(6) & 0.3526(3) & 0.0922(19)^{*} \\ \text { C3S } & -0.0383(7) & 0.7913(9) & 0.4094(4) & 0.104(2)^{*} \\ \text { C4S } & 0.0152(8) & 0.6288(9) & 0.4244(5) & 0.149(4)^{*}\end{array}$

Anisotropically-refined atoms are marked with an asterisk $(*)$. The form of the anisotropic displacement parameter is: $\exp \left[-2 \pi^{2}\left(h^{2} a^{* 2} U_{11}+k^{2} b^{* 2} U_{22}+l^{2} c^{* 2} U_{33}+2 k l b^{*} c^{*} U_{23}\right.\right.$ $\left.\left.+2 h l a^{*} c^{*} U_{13}+2 h k a^{*} b^{*} U_{12}\right)\right]$. 
Table 3. Selected Interatomic Distances ( $(̊)$

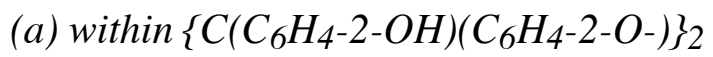

$\begin{array}{llllll}\text { Atom1 } & \text { Atom2 } & \text { Distance } & \text { Atom1 } & \text { Atom2 } & \text { Distance } \\ \text { O1 } & \text { C2 } & 1.465(4) & \text { C21 } & \text { C22 } & 1.392(5) \\ \text { O1 } & \text { C12 } & 1.397(4) & \text { C21 } & \text { C26 } & 1.385(5) \\ \text { O1 } & \text { H4 } & 1.86^{\dagger} & \text { C22 } & \text { C23 } & 1.382(5) \\ \text { O2 } & \text { C22 } & 1.375(4) & \text { C23 } & \text { C24 } & 1.382(5) \\ \text { O3 } & \text { C1 } & 1.466(4) & \text { C24 } & \text { C25 } & 1.381(5) \\ \text { O3 } & \text { C32 } & 1.369(4) & \text { C25 } & \text { C26 } & 1.387(5) \\ \text { O4 } & \text { C42 } & 1.360(4) & \text { C31 } & \text { C32 } & 1.374(5) \\ \text { C1 } & \text { C2 } & 1.605(5) & \mathrm{C} 31 & \mathrm{C} 36 & 1.385(5) \\ \text { C1 } & \text { C11 } & 1.509(5) & \mathrm{C} 32 & \mathrm{C} 33 & 1.388(5) \\ \text { C1 } & \text { C21 } & 1.524(5) & \mathrm{C} 33 & \mathrm{C} 34 & 1.382(5) \\ \mathrm{C} 2 & \mathrm{C} 31 & 1.504(5) & \mathrm{C} 34 & \mathrm{C} 35 & 1.390(6) \\ \mathrm{C} 2 & \mathrm{C} 41 & 1.527(5) & \mathrm{C} 35 & \mathrm{C} 36 & 1.391(5) \\ \mathrm{C} 11 & \mathrm{C} 12 & 1.370(5) & \mathrm{C} 41 & \mathrm{C} 42 & 1.388(5) \\ \mathrm{C} 11 & \mathrm{C} 16 & 1.385(5) & \mathrm{C} 41 & \mathrm{C} 46 & 1.414(5) \\ \mathrm{C} 12 & \mathrm{C} 13 & 1.376(5) & \mathrm{C} 42 & \mathrm{C} 43 & 1.387(5) \\ \mathrm{C} 13 & \mathrm{C} 14 & 1.382(6) & \mathrm{C} 43 & \mathrm{C} 44 & 1.367(6) \\ \mathrm{C} 14 & \mathrm{C} 15 & 1.387(6) & \mathrm{C} 44 & \mathrm{C} 45 & 1.382(5) \\ \mathrm{C} 15 & \mathrm{C} 16 & 1.378(6) & \mathrm{C} 45 & \mathrm{C} 46 & 1.398(5)\end{array}$

(b) within the solvent diethyl ether molecule

$\begin{array}{llllll}\text { Atom1 } & \text { Atom2 } & \text { Distance } & \text { Atom1 } & \text { Atom2 } & \text { Distance } \\ \text { O1S } & \text { C1S } & 1.438(6) & \text { C1S } & \text { C2S } & 1.454(7) \\ \text { O1S } & \text { C3S } & 1.425(5) & \text { C3S } & \text { C4S } & 1.457(8)\end{array}$

$\begin{array}{lll}\mathrm{O} 1 \mathrm{~S} & \mathrm{H} 2 & 1.86^{\dagger}\end{array}$

$\nmid$ Nonbonded distance. 
Table 4. Selected Interatomic Angles (deg)

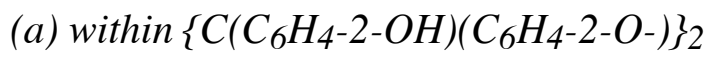

$\begin{array}{llllllll}\text { Atom1 } & \text { Atom2 } & \text { Atom3 } & \text { Angle } & \text { Atom1 } & \text { Atom2 } & \text { Atom3 } & \text { Angle } \\ \text { C2 } & \text { O1 } & \text { C12 } & 107.8(3) & \text { O2 } & \text { C22 } & \text { C21 } & 117.2(4) \\ \text { C1 } & \text { O3 } & \text { C32 } & 108.0(3) & \text { O2 } & \text { C22 } & \text { C23 } & 121.8(4) \\ \text { O3 } & \text { C1 } & \text { C2 } & 106.0(3) & \text { C21 } & \text { C22 } & \text { C23 } & 120.9(4) \\ \text { O3 } & \text { C1 } & \text { C11 } & 109.8(3) & \text { C22 } & \text { C23 } & \text { C24 } & 119.4(4) \\ \text { O3 } & \text { C1 } & \text { C21 } & 107.0(3) & \text { C23 } & \text { C24 } & \text { C25 } & 120.7(4) \\ \text { C2 } & \text { C1 } & \text { C11 } & 101.0(3) & \text { C24 } & \text { C25 } & \text { C26 } & 119.3(4) \\ \text { C2 } & \text { C1 } & \text { C21 } & 118.3(3) & \text { C21 } & \text { C26 } & \text { C25 } & 121.0(4) \\ \text { C11 } & \text { C1 } & \text { C21 } & 114.3(3) & \text { C2 } & \text { C31 } & \text { C32 } & 110.0(3) \\ \text { O1 } & \text { C2 } & \text { C1 } & 106.4(3) & \text { C2 } & \text { C31 } & \text { C36 } & 130.4(4) \\ \text { O1 } & \text { C2 } & \text { C31 } & 109.3(3) & \text { C32 } & \text { C31 } & \text { C36 } & 119.6(4) \\ \text { O1 } & \text { C2 } & \text { C41 } & 109.2(3) & \text { O3 } & \text { C32 } & \text { C31 } & 114.2(4) \\ \text { C1 } & \text { C2 } & \text { C31 } & 100.8(3) & \text { O3 } & \text { C32 } & \text { C33 } & 122.7(4) \\ \text { C1 } & \text { C2 } & \text { C41 } & 117.8(3) & \text { C31 } & \text { C32 } & \text { C33 } & 123.1(4) \\ \text { C31 } & \text { C2 } & \text { C41 } & 112.7(3) & \text { C32 } & \text { C33 } & \text { C34 } & 116.3(4) \\ \text { C1 } & \text { C11 } & \text { C12 } & 110.4(4) & \text { C33 } & \text { C34 } & \text { C35 } & 122.1(4) \\ \text { C1 } & \text { C11 } & \text { C16 } & 129.8(4) & \text { C34 } & \text { C35 } & \text { C36 } & 119.8(4) \\ \text { C12 } & \text { C11 } & \text { C16 } & 119.8(4) & \text { C31 } & \text { C36 } & \text { C35 } & 119.0(4) \\ \text { O1 } & \text { C12 } & \text { C11 } & 113.5(4) & \text { C2 } & \text { C41 } & \text { C42 } & 126.0(4) \\ \text { O1 } & \text { C12 } & \text { C13 } & 123.1(4) & \text { C2 } & \text { C41 } & \text { C46 } & 116.3(4) \\ \text { C11 } & \text { C12 } & \text { C13 } & 123.2(4) & \text { C42 } & \text { C41 } & \text { C46 } & 117.7(4) \\ \text { C12 } & \text { C13 } & \text { C14 } & 116.6(5) & \text { O4 } & \text { C42 } & \text { C41 } & 124.4(4) \\ \text { C13 } & \text { C14 } & \text { C15 } & 121.1(5) & \text { O4 } & \text { C42 } & \text { C43 } & 113.9(4) \\ \text { C14 } & \text { C15 } & \text { C16 } & 121.1(5) & \text { C41 } & \text { C42 } & \text { C43 } & 121.7(4) \\ \text { C11 } & \text { C16 } & \text { C15 } & 118.1(5) & \text { C42 } & \text { C43 } & \text { C44 } & 119.9(4) \\ \text { C1 } & \text { C21 } & \text { C22 } & 119.8(4) & \text { C43 } & \text { C44 } & \text { C45 } & 120.6(4) \\ \text { C1 } & \text { C21 } & \text { C26 } & 121.6(4) & \text { C44 } & \text { C45 } & \text { C46 } & 119.9(4) \\ \text { C22 } & \text { C21 } & \text { C26 } & 118.6(4) & \text { C41 } & \text { C46 } & \text { C45 } & 120.2(4)\end{array}$

(b) within the solvent diethyl ether molecule

$\begin{array}{llllllll}\text { Atom1 } & \text { Atom2 } & \text { Atom3 } & \text { Angle } & \text { Atom1 } & \text { Atom2 } & \text { Atom3 } & \text { Angle } \\ \text { C1S } & \text { O1S } & \text { C3S } & 114.1(5) & \text { O1S } & \text { C3S } & \text { C4S } & 110.2(5) \\ \text { O1S } & \text { C1S } & \text { C2S } & 110.7(4) & & & & \end{array}$


Table 5. Anisotropic Displacement Parameters $\left(U_{\mathrm{ij}}, \AA^{2}\right)$

\begin{tabular}{|c|c|c|c|c|c|c|}
\hline Atom & $U_{11}$ & $U_{22}$ & $U_{33}$ & $U_{23}$ & $U_{13}$ & $U_{12}$ \\
\hline $\mathrm{O} 1$ & $0.0445(17)$ & $0.0421(18)$ & $0.0308(19)$ & $0.0003(15)$ & $-0.0126(15)$ & $-0.0151(15)$ \\
\hline $\mathrm{O} 2$ & $0.0408(17)$ & $0.0368(19)$ & $0.049(2)$ & $0.0004(16)$ & $-0.0209(16)$ & $-0.0045(15)$ \\
\hline $\mathrm{O} 3$ & $0.0376(17)$ & $0.0353(17)$ & $0.045(2)$ & $-0.0014(15)$ & $-0.0066(16)$ & $-0.0134(14)$ \\
\hline $\mathrm{O} 4$ & $0.089(3)$ & $0.062(2)$ & $0.052(2)$ & $-0.0057(19)$ & $-0.016(2)$ & $-0.034(2)$ \\
\hline $\mathrm{C} 1$ & $0.027(2)$ & $0.026(2)$ & $0.046(3)$ & $-0.001(2)$ & $-0.005(2)$ & $-0.007(2)$ \\
\hline $\mathrm{C} 2$ & $0.032(2)$ & $0.027(2)$ & $0.028(3)$ & $0.000(2)$ & $-0.008(2)$ & $-0.011(2)$ \\
\hline C11 & $0.030(2)$ & $0.026(2)$ & $0.036(3)$ & $0.007(2)$ & $-0.005(2)$ & $-0.004(2)$ \\
\hline $\mathrm{C} 12$ & $0.036(3)$ & $0.031(3)$ & $0.035(3)$ & $0.008(2)$ & $-0.005(2)$ & $-0.004(2)$ \\
\hline C13 & $0.051(3)$ & $0.046(3)$ & $0.031(3)$ & $0.005(3)$ & $-0.003(3)$ & $-0.004(3)$ \\
\hline C14 & $0.056(3)$ & $0.054(3)$ & $0.037(3)$ & $0.013(3)$ & $-0.003(3)$ & $-0.002(3)$ \\
\hline C15 & $0.049(3)$ & $0.052(4)$ & $0.060(4)$ & $0.017(3)$ & $0.010(3)$ & $-0.009(3)$ \\
\hline C16 & $0.045(3)$ & $0.041(3)$ & $0.058(4)$ & $0.011(3)$ & $-0.006(3)$ & $-0.017(2)$ \\
\hline $\mathrm{C} 21$ & $0.039(2)$ & $0.032(3)$ & $0.037(3)$ & $0.007(2)$ & $-0.012(2)$ & $-0.022(2)$ \\
\hline $\mathrm{C} 22$ & $0.034(2)$ & $0.039(3)$ & $0.041(3)$ & $0.006(2)$ & $-0.016(2)$ & $-0.016(2)$ \\
\hline $\mathrm{C} 23$ & $0.046(3)$ & $0.038(3)$ & $0.049(3)$ & $0.010(2)$ & $-0.023(3)$ & $-0.018(2)$ \\
\hline C24 & $0.044(3)$ & $0.046(3)$ & $0.067(4)$ & $0.022(3)$ & $-0.030(3)$ & $-0.026(3)$ \\
\hline $\mathrm{C} 25$ & $0.052(3)$ & $0.061(3)$ & $0.047(3)$ & $0.006(3)$ & $-0.025(3)$ & $-0.034(3)$ \\
\hline $\mathrm{C} 26$ & $0.043(3)$ & $0.043(3)$ & $0.045(3)$ & $0.005(2)$ & $-0.009(2)$ & $-0.026(2)$ \\
\hline C31 & $0.031(2)$ & $0.026(2)$ & $0.033(3)$ & $0.009(2)$ & $-0.011(2)$ & $-0.010(2)$ \\
\hline C32 & $0.029(2)$ & $0.037(3)$ & $0.041(3)$ & $0.009(2)$ & $-0.004(2)$ & $-0.008(2)$ \\
\hline C33 & $0.045(3)$ & $0.030(3)$ & $0.057(3)$ & $0.005(3)$ & $-0.010(3)$ & $-0.011(2)$ \\
\hline C34 & $0.044(3)$ & $0.034(3)$ & $0.064(4)$ & $0.010(3)$ & $-0.003(3)$ & $0.001(2)$ \\
\hline C35 & $0.034(3)$ & $0.039(3)$ & $0.069(4)$ & $0.016(3)$ & $-0.007(3)$ & $-0.008(2)$ \\
\hline C36 & $0.037(3)$ & $0.035(3)$ & $0.053(3)$ & $0.014(3)$ & $-0.018(2)$ & $-0.012(2)$ \\
\hline C41 & $0.026(2)$ & $0.033(3)$ & $0.035(3)$ & $0.003(2)$ & $-0.013(2)$ & $-0.014(2)$ \\
\hline C42 & $0.034(2)$ & $0.043(3)$ & $0.032(3)$ & $-0.009(3)$ & $-0.006(2)$ & $-0.016(2)$ \\
\hline $\mathrm{C} 43$ & $0.047(3)$ & $0.027(3)$ & $0.059(4)$ & $-0.006(3)$ & $-0.008(3)$ & $-0.017(2)$ \\
\hline C44 & $0.037(3)$ & $0.031(3)$ & $0.081(4)$ & $0.017(3)$ & $-0.022(3)$ & $-0.017(2)$ \\
\hline $\mathrm{C} 45$ & $0.040(3)$ & $0.039(3)$ & $0.040(3)$ & $0.010(2)$ & $-0.016(2)$ & $-0.014(2)$ \\
\hline C46 & $0.033(2)$ & $0.038(3)$ & $0.041(3)$ & $0.009(2)$ & $-0.014(2)$ & $-0.016(2)$ \\
\hline O1S & $0.0392(18)$ & $0.063(2)$ & $0.047(2)$ & 0.0091(18) & $-0.0093(16)$ & $-0.0093(17)$ \\
\hline C1S & $0.084(4)$ & $0.053(4)$ & $0.094(5)$ & $-0.013(4)$ & $-0.054(4)$ & $0.009(3)$ \\
\hline $\mathrm{C} 2 \mathrm{~S}$ & $0.140(6)$ & $0.053(4)$ & $0.085(5)$ & $0.023(3)$ & $-0.042(4)$ & $-0.038(4)$ \\
\hline C3S & $0.046(3)$ & $0.180(7)$ & $0.076(5)$ & $-0.039(5)$ & $0.010(3)$ & $-0.053(5)$ \\
\hline $\mathrm{C} 4 \mathrm{~S}$ & $0.131(6)$ & $0.222(9)$ & $0.193(8)$ & $0.152(7)$ & $-0.102(6)$ & $-0.141(7)$ \\
\hline
\end{tabular}

The form of the anisotropic displacement parameter is:

$\exp \left[-2 \pi^{2}\left(h^{2} a^{* 2} U_{11}+k^{2} b^{* 2} U_{22}+l^{2} c^{* 2} U_{33}+2 k l b^{*} c^{*} U_{23}+2 h l a^{*} c^{*} U_{13}+2 h k a^{*} b^{*} U_{12}\right)\right]$ 
Table 6. Derived Atomic Coordinates and Displacement Parameters for Hydrogen Atoms

$\begin{array}{lcccc}\text { Atom } & x & y & z & U_{\text {eq }} \AA^{2} \\ \text { H2 } & 0.3187 & 0.6706 & 0.3268 & 0.067 \\ \text { H4 } & 0.7311 & 0.6229 & 0.3441 & 0.101 \\ \text { H13 } & 0.7034 & 0.3636 & 0.4804 & 0.060 \\ \text { H14 } & 0.5345 & 0.2298 & 0.5344 & 0.070 \\ \text { H15 } & 0.4202 & 0.1385 & 0.4481 & 0.077 \\ \text { H16 } & 0.4760 & 0.1722 & 0.3054 & 0.060 \\ \text { H23 } & 0.1720 & 0.7290 & 0.2247 & 0.051 \\ \text { H24 } & 0.1586 & 0.6336 & 0.0999 & 0.056 \\ \text { H25 } & 0.3856 & 0.4068 & 0.0236 & 0.057 \\ \text { H26 } & 0.6211 & 0.2683 & 0.0763 & 0.050 \\ \text { H33 } & 1.0870 & -0.0684 & 0.0876 & 0.056 \\ \text { H34 } & 1.3667 & -0.0906 & 0.0750 & 0.068 \\ \text { H35 } & 1.4090 & 0.1071 & 0.1310 & 0.062 \\ \text { H36 } & 1.1683 & 0.3347 & 0.2018 & 0.050 \\ \text { H43 } & 0.7082 & 0.9131 & 0.2332 & 0.054 \\ \text { H44 } & 0.7611 & 0.9072 & 0.0889 & 0.058 \\ \text { H45 } & 0.8244 & 0.6821 & 0.0085 & 0.048 \\ \text { H46 } & 0.8472 & 0.4559 & 0.0731 & 0.044 \\ \text { H1SA } & -0.0200 & 1.0558 & 0.4213 & 0.101 \\ \text { H1SB } & 0.0178 & 1.0067 & 0.3237 & 0.101 \\ \text { H2SA } & 0.1931 & 1.1196 & 0.3409 & 0.111 \\ \text { H2SB } & 0.3094 & 0.9419 & 0.3033 & 0.111 \\ \text { H2SC } & 0.2712 & 0.9916 & 0.4007 & 0.111 \\ \text { H3SA } & -0.0923 & 0.8125 & 0.3627 & 0.125 \\ \text { H3SB } & -0.1258 & 0.8583 & 0.4603 & 0.125 \\ \text { H4SA } & -0.0861 & 0.6056 & 0.4376 & 0.179 \\ \text { H4SB } & 0.0658 & 0.6088 & 0.4716 & 0.179 \\ \text { H4SC } & 0.1018 & 0.5628 & 0.3740 & 0.179\end{array}$




\section{X-Ray Crystal Structure Report}

Compound: Mono-Sodium Salt of Z-Bis(5-tert-butyl-2-hydroxyphenyl)-bis(5-tert-butyl-2methoxyphenyl)ethene

$\left[\left(\mathrm{C}_{4} \mathrm{H}_{8} \mathrm{O}\right) \mathrm{Na}\left\{\kappa^{4}-\mathrm{C}_{2}\left(\mathrm{C}_{6} \mathrm{H}_{3}{ }^{t} \mathrm{BuOM} \underline{\mathrm{OM}}\right)_{2}\left(\mathrm{C}_{6} \mathrm{H}_{3}{ }^{t} \mathrm{BuOH}\right)\left(\mathrm{C}_{6} \mathrm{H}_{3}{ }^{t} \mathrm{BuO}\right)\right] \bullet 0.25 n-\mathrm{C}_{6} \mathrm{H}_{14}\right.$

Formula: $\quad \mathrm{C}_{49.5} \mathrm{H}_{66.5} \mathrm{NaO}_{5}\left(\mathrm{C}_{48} \mathrm{H}_{63} \mathrm{NaO}_{5} \bullet 0.25 \mathrm{C}_{6} \mathrm{H}_{14}\right)$

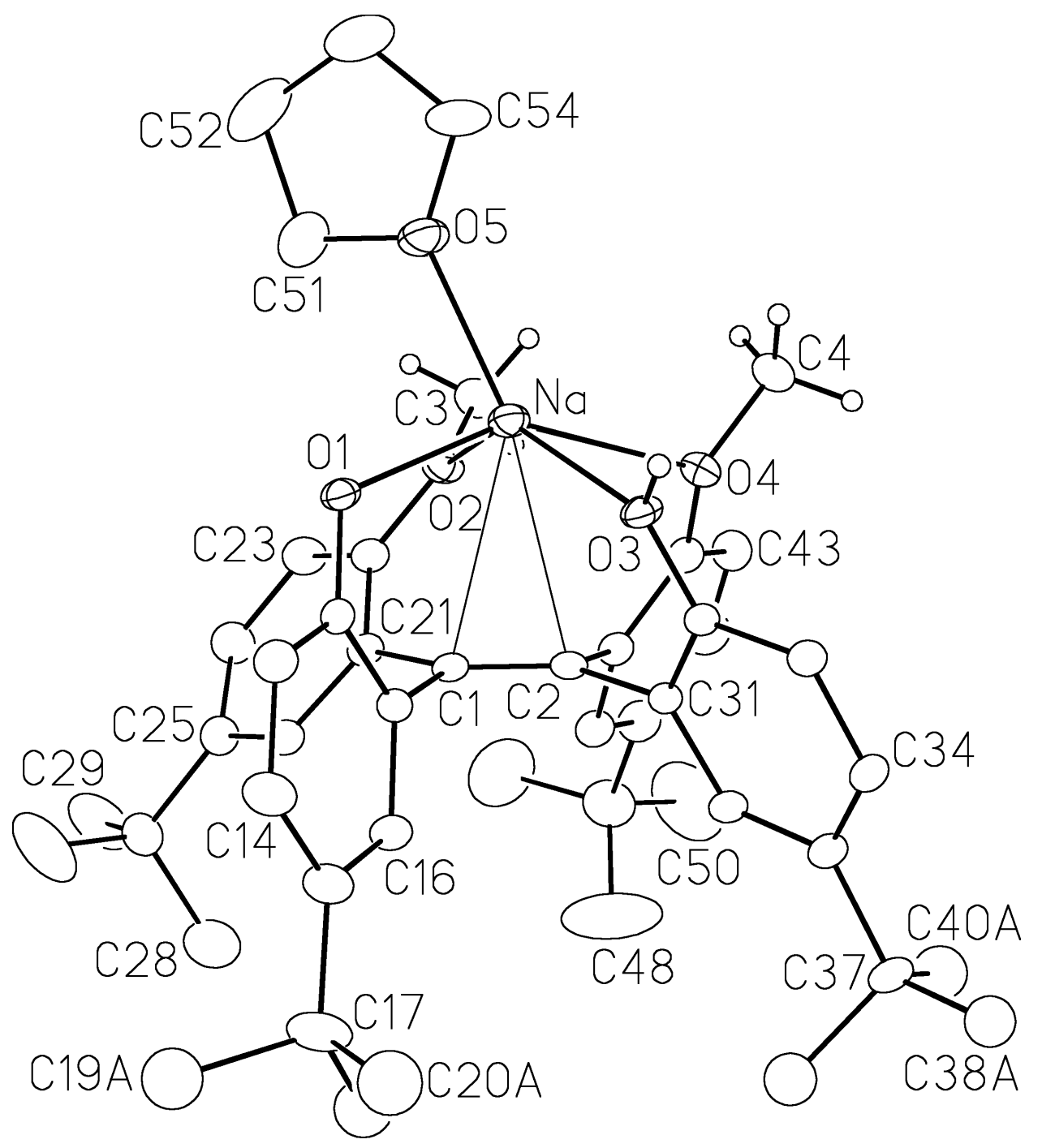

Figure 1. Perspective view of the $\left[\left(\mathrm{C}_{4} \mathrm{H}_{8} \mathrm{O}\right) \mathrm{Na}\left\{\kappa^{4}-\mathrm{C}_{2}\left(\mathrm{C}_{6} \mathrm{H}_{3}{ }^{t} \mathrm{BuOMe}\right)_{2}\left(\mathrm{C}_{6} \mathrm{H}_{3}{ }^{t} \mathrm{BuOH}\right)\right.\right.$ $\left.\left(\mathrm{C}_{6} \mathrm{H}_{3}{ }^{t} \mathrm{BuO}\right)\right]$ molecule showing the atom labelling scheme. Non-hydrogen atoms are represented by Gaussian ellipsoids at the $20 \%$ probability level. Methoxy and hydroxyl hydrogen atoms are shown with arbitrarily small thermal parameters; all other hydrogens are not shown. 


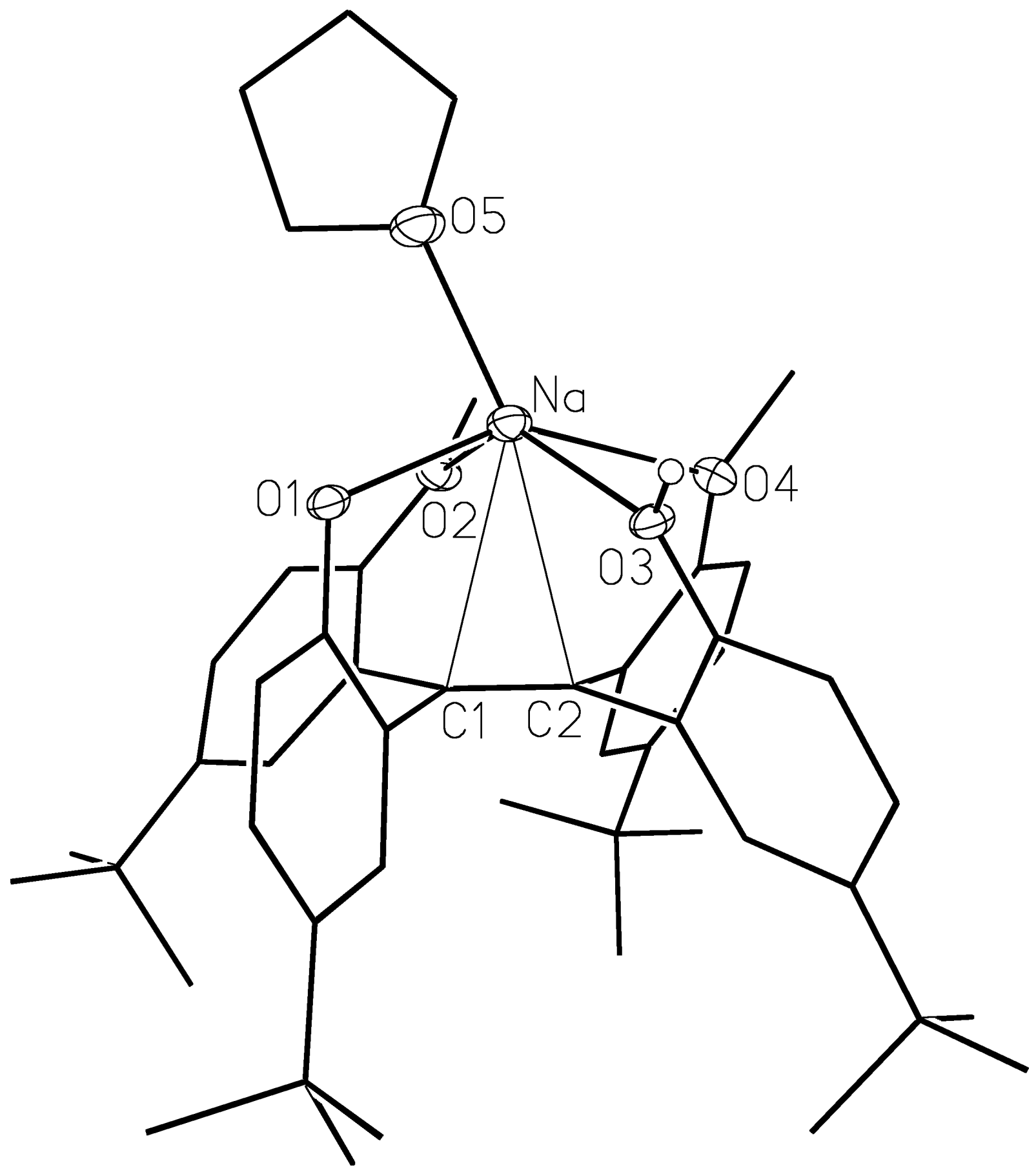

Figure 2. View as in Figure 1, with carbon displacement ellipsoids and methyl hydrogen positions omitted. 


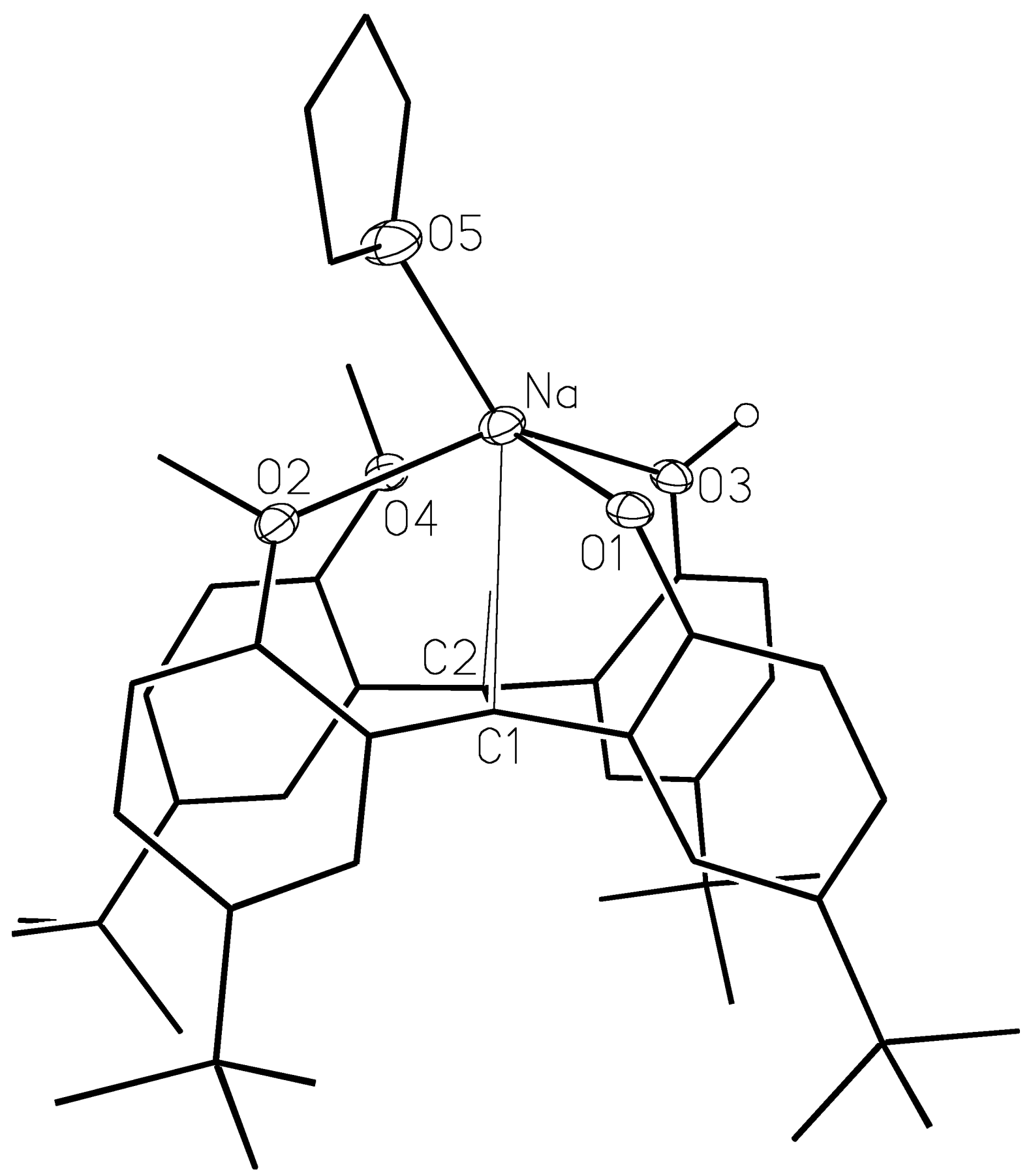

Figure 3. View of the molecule approximately along the $\mathrm{C} 1-\mathrm{C} 2$ bond. 


\section{List of Tables}

Table 1. Crystallographic Experimental Details

Table 2. Atomic Coordinates and Equivalent Isotropic Displacement Parameters

Table 3. Selected Interatomic Distances

Table 4. Selected Interatomic Angles

Table 5. Anisotropic Displacement Parameters

Table 6. Derived Atomic Coordinates and Displacement Parameters for Hydrogen Atoms

Table 1. Crystallographic Experimental Details

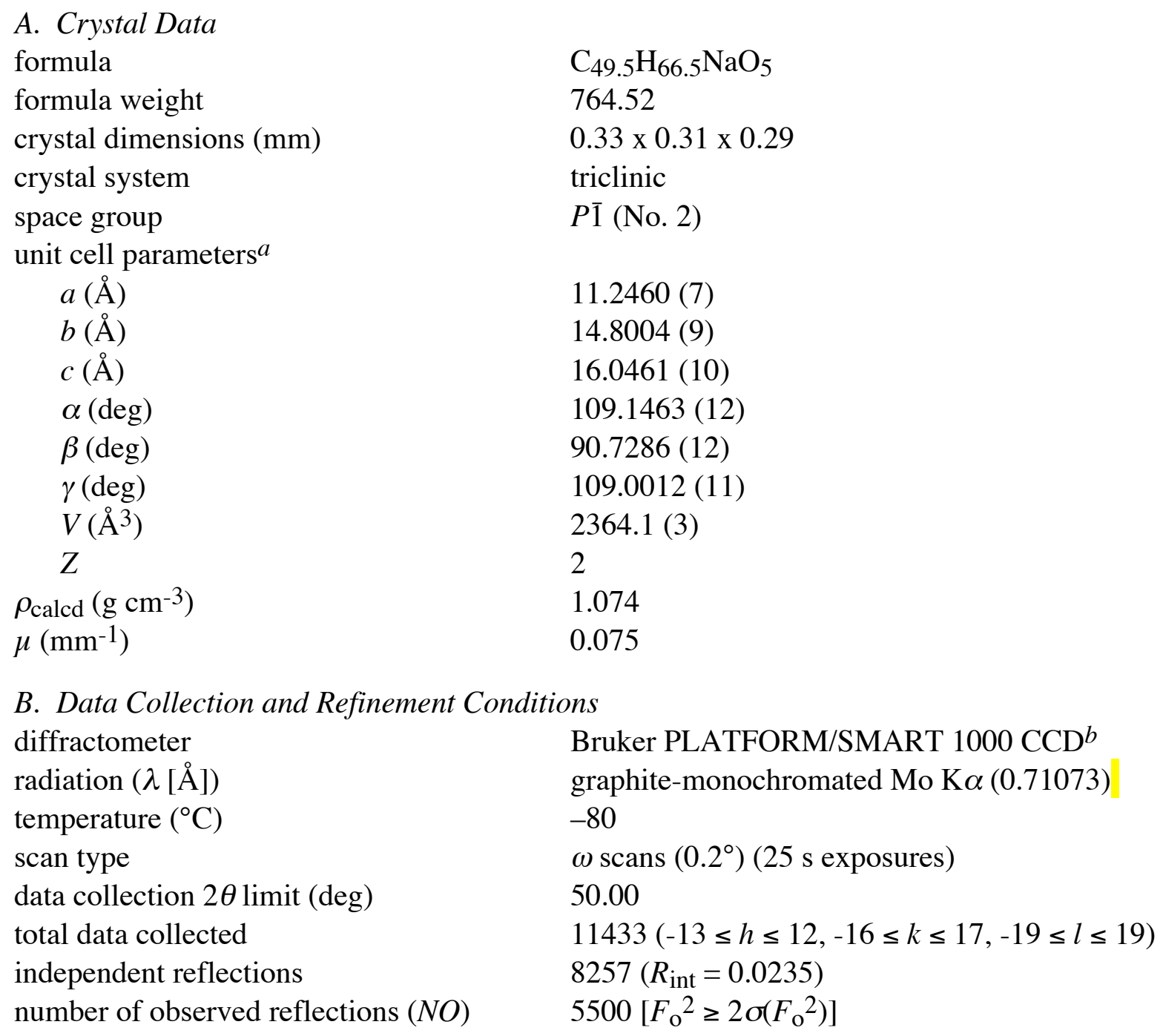

$\begin{array}{ll}a(\AA) & 11.2460(7) \\ b(\AA) & 14.8004(9) \\ c(\AA) & 16.0461(10) \\ \alpha(\mathrm{deg}) & 109.1463(12) \\ \beta(\mathrm{deg}) & 90.7286(12) \\ \gamma(\mathrm{deg}) & 109.0012(11) \\ V\left(\AA^{3}\right) & 2364.1(3) \\ Z & 2 \\ \rho_{\text {calcd }}\left(\mathrm{g} \mathrm{cm}^{-3}\right) & 1.074 \\ \left(\mathrm{~mm}^{-1}\right) & 0.075\end{array}$

B. Data Collection and Refinement Conditions diffractometer radiation $(\lambda[\AA]])$ Bruker PLATFORM/SMART $1000 \mathrm{CCD}^{b}$

temperature $\left({ }^{\circ} \mathrm{C}\right)$ graphite-monochromated Mo $\mathrm{K} \alpha(0.71073)$ scan type $-80$ data collection $2 \theta$ limit (deg) $\quad 50.00$ total data collected $11433(-13 \leq h \leq 12,-16 \leq k \leq 17,-19 \leq l \leq 19)$ independent reflections $8257\left(R_{\text {int }}=0.0235\right)$ number of observed reflections $(N O) \quad 5500\left[F_{\mathrm{o}}{ }^{2} \geq 2 \sigma\left(F_{\mathrm{O}}{ }^{2}\right)\right]$ 
structure solution method

refinement method

absorption correction method

range of transmission factors

data/restraints/parameters

goodness-of-fit $(S)^{f}$

final $R$ indices $g$

$$
\begin{aligned}
& R_{1}\left[F_{\mathrm{O}}^{2} \geq 2 \sigma\left(F_{\mathrm{O}}^{2}\right)\right] \\
& w R_{2}\left[F_{\mathrm{O}}^{2} \geq-3 \sigma\left(F_{\mathrm{O}}^{2}\right)\right]
\end{aligned}
$$

largest difference peak and hole direct methods (SHELXS-86 $)$

full-matrix least-squares on $F^{2}\left(S H E L X L-93^{d}\right)$

Gaussian integration (face-indexed)

0.9785-0.9756

$8257\left[F_{\mathrm{O}}^{2} \geq-3 \sigma\left(F_{\mathrm{O}}^{2}\right)\right] / 5^{e} / 491$

$1.029\left[F_{\mathrm{O}}^{2} \geq-3 \sigma\left(F_{\mathrm{O}}^{2}\right)\right]$

0.0938

0.2980

0.842 and -0.398 e $\AA^{-3}$

${ }^{a}$ Obtained from least-squares refinement of 3926 reflections with $4.52^{\circ}<2 \theta<48.31^{\circ}$.

${ }^{b}$ Programs for diffractometer operation, data collection, data reduction and absorption correction were those supplied by Bruker.

cSheldrick, G. M. Acta Crystallogr. 1990, A46, 467-473.

${ }^{d}$ Sheldrick, G. M. SHELXL-93. Program for crystal structure determination. University of Göttingen, Germany, 1993. Refinement on $F_{\mathrm{O}}{ }^{2}$ for all reflections (all of these having $F_{\mathrm{O}}{ }^{2} \geq$ $\left.-3 \sigma\left(F_{\mathrm{o}}^{2}\right)\right)$. Weighted $R$-factors $w R_{2}$ and all goodnesses of fit $S$ are based on $F_{\mathrm{o}}^{2}$; conventional $R$-factors $R_{1}$ are based on $F_{\mathrm{o}}$, with $F_{\mathrm{O}}$ set to zero for negative $F_{\mathrm{O}}{ }^{2}$. The observed criterion of $F_{\mathrm{O}}{ }^{2}>2 \sigma\left(F_{\mathrm{O}}{ }^{2}\right)$ is used only for calculating $R_{1}$, and is not relevant to the choice of reflections for refinement. $R$-factors based on $F_{\mathrm{O}}{ }^{2}$ are statistically about twice as large as those based on $F_{\mathrm{O}}$, and $R$-factors based on ALL data will be even larger.

${ }^{e}$ An idealized geometry was imposed upon the solvent n-hexan molecule though use of the following interatomic distance restraints: $\mathrm{d}\left(\mathrm{C} 1 \mathrm{~S}-\mathrm{C} 1 \mathrm{~S}^{\prime}\right)=\mathrm{d}(\mathrm{C} 1 \mathrm{~S}-\mathrm{C} 2 \mathrm{~S})=\mathrm{d}(\mathrm{C} 2 \mathrm{~S}-\mathrm{C} 3 \mathrm{~S})=1.54$ $\AA ; \mathrm{d}\left(\mathrm{C} 1 \mathrm{~S} \cdots \mathrm{C} 2 \mathrm{~S}^{\prime}\right)=\mathrm{d}(\mathrm{C} 1 \mathrm{~S} \cdots \mathrm{C} 3 \mathrm{~S})=2.52 \AA$ (primed atoms are related to unprimed ones via the crystallographic inversion center $(0,1 / 2,0)$ at the midpoint of the $\mathrm{C} 1 \mathrm{~S}-\mathrm{C} 1 \mathrm{~S}$ ' bond).

$f_{S}=\left[\Sigma w\left(F_{\mathrm{o}}{ }^{2}-F_{\mathrm{c}^{2}}\right)^{2} /(n-p)\right]^{1 / 2}(n=$ number of data; $p=$ number of parameters varied; $w=$ $\left[\sigma^{2}\left(F_{\mathrm{o}}^{2}\right)+(0.1595 P)^{2}+3.0444 P\right]^{-1}$ where $\left.P=\left[\operatorname{Max}\left(F_{\mathrm{o}}^{2}, 0\right)+2 F_{\mathrm{c}}^{2}\right] / 3\right)$.

$g R_{1}=\Sigma|| F_{\mathrm{o}}|-| F_{\mathrm{c}}|| / \Sigma\left|F_{\mathrm{o}}\right| ; w R_{2}=\left[\Sigma w\left(F_{\mathrm{o}}{ }^{2}-F_{\mathrm{c}^{2}}\right)^{2} / \Sigma w\left(F_{\mathrm{o}}{ }^{4}\right)\right]^{1 / 2}$. 
Table 2. Atomic Coordinates and Equivalent Isotropic Displacement Parameters (a) atoms of $\left[\left(\mathrm{C}_{4} \mathrm{H}_{8} \mathrm{O}\right) \mathrm{Na}\left\{\mathrm{K}^{4}-\mathrm{C}_{2}\left(\mathrm{C}_{6} \mathrm{H}_{3}{ }^{t} \mathrm{Bu} \underline{\mathrm{OMe}}\right)_{2}\left(\mathrm{C}_{6} \mathrm{H}_{3}{ }^{t} \mathrm{Bu} \underline{\mathrm{OH}}\right)\left(\mathrm{C}_{6} \mathrm{H}_{3}{ }^{t} \mathrm{Bu} \underline{\mathrm{O}}\right)\right]\right.$

\begin{tabular}{|c|c|c|c|c|}
\hline Atom & $x$ & $y$ & $z$ & $U_{\text {eq }}, \AA^{2}$ \\
\hline $\mathrm{Na}$ & $0.41550(15)$ & $0.12404(11)$ & $-0.00961(10)$ & $0.0475(4)^{*}$ \\
\hline $\mathrm{O} 1$ & $0.6171(2)$ & $0.16454(19)$ & $0.06206(16)$ & $0.0407(6)^{*}$ \\
\hline $\mathrm{O} 2$ & $0.3942(3)$ & $0.2941(2)$ & $0.03552(17)$ & $0.0493(7) *$ \\
\hline $\mathrm{O} 3$ & $0.3476(2)$ & $-0.01206(19)$ & $0.03594(16)$ & $0.0418(6)^{*}$ \\
\hline $\mathrm{O} 4$ & $0.1668(3)$ & $0.1001(2)$ & $0.00205(17)$ & $0.0470(7)^{*}$ \\
\hline $\mathrm{O} 5$ & $0.4783(4)$ & $0.1738(3)$ & $-0.1317(2)$ & $0.0733(10)^{*}$ \\
\hline $\mathrm{C} 1$ & $0.4471(3)$ & $0.2314(3)$ & $0.1781(2)$ & $0.0348(8) *$ \\
\hline $\mathrm{C} 2$ & $0.3237(3)$ & $0.1740(3)$ & $0.1622(2)$ & $0.0351(8)^{*}$ \\
\hline $\mathrm{C} 3$ & $0.3211(5)$ & $0.3194(4)$ & $-0.0206(3)$ & $0.0624(12)^{*}$ \\
\hline $\mathrm{C} 4$ & $0.0830(4)$ & $0.0541(4)$ & $-0.0804(3)$ & $0.0563(11)^{*}$ \\
\hline C11 & $0.5476(3)$ & $0.1964(3)$ & $0.2053(2)$ & $0.0359(8)^{*}$ \\
\hline $\mathrm{C} 12$ & $0.6303(3)$ & $0.1671(3)$ & $0.1459(2)$ & $0.0369(8)^{*}$ \\
\hline C13 & $0.7249(4)$ & $0.1410(3)$ & $0.1782(3)$ & $0.0498(10)^{*}$ \\
\hline $\mathrm{C} 14$ & $0.7401(4)$ & $0.1456(4)$ & $0.2653(3)$ & $0.0585(12)^{*}$ \\
\hline $\mathrm{C} 15$ & $0.6598(4)$ & $0.1752(3)$ & $0.3255(3)$ & $0.0521(10)^{*}$ \\
\hline $\mathrm{C} 16$ & $0.5627(4)$ & $0.1983(3)$ & $0.2925(2)$ & $0.0415(9)^{*}$ \\
\hline $\mathrm{C} 17$ & $0.6774(6)$ & $0.1841(4)$ & $0.4231(3)$ & $0.0714(15)^{*}$ \\
\hline $\mathrm{C}_{18 \mathrm{~A}^{a}}$ & $0.6008(11)$ & $0.2333(9)$ & $0.4830(8)$ & $0.094(3)$ \\
\hline $\mathrm{C}_{19 \mathrm{~A}^{a}}$ & $0.8192(10)$ & $0.2499(9)$ & $0.4681(8)$ & $0.096(3)$ \\
\hline $\mathrm{C} 20 \mathrm{~A}^{a}$ & $0.6595(12)$ & $0.0800(9)$ & $0.4327(8)$ & $0.102(3)$ \\
\hline $\mathrm{C} 18 \mathrm{~B}^{b}$ & $0.5397(14)$ & $0.1201(12)$ & $0.4433(11)$ & $0.085(4)$ \\
\hline $\mathrm{C} 19 \mathrm{~B}^{b}$ & $0.7056(16)$ & $0.2935(12)$ & $0.4794(11)$ & $0.086(4)$ \\
\hline $\mathrm{C} 20 \mathrm{~B}^{b}$ & $0.7680(17)$ & $0.1378(14)$ & $0.4398(12)$ & $0.093(4)$ \\
\hline $\mathrm{C} 21$ & $0.4908(4)$ & $0.3402(3)$ & $0.1820(2)$ & $0.0389(8)^{*}$ \\
\hline $\mathrm{C} 22$ & $0.4565(4)$ & $0.3703(3)$ & $0.1140(3)$ & $0.0456(9)^{*}$ \\
\hline $\mathrm{C} 23$ & $0.4867(5)$ & $0.4729(3)$ & $0.1274(3)$ & $0.0599(12)^{*}$ \\
\hline $\mathrm{C} 24$ & $0.5528(5)$ & $0.5467(3)$ & $0.2068(3)$ & $0.0615(12)^{*}$ \\
\hline $\mathrm{C} 25$ & $0.5931(4)$ & $0.5207(3)$ & $0.2755(3)$ & $0.0528(10)^{*}$ \\
\hline $\mathrm{C} 26$ & $0.5619(4)$ & $0.4167(3)$ & $0.2601(3)$ & $0.0440(9)^{*}$ \\
\hline $\mathrm{C} 27$ & $0.6638(5)$ & $0.6009(3)$ & $0.3647(3)$ & $0.0610(12)^{*}$ \\
\hline $\mathrm{C} 28$ & $0.5895(8)$ & $0.5797(6)$ & $0.4401(4)$ & $0.117(3)^{*}$ \\
\hline C29 & $0.6684(8)$ & $0.7079(5)$ & $0.3728(5)$ & $0.116(3)^{*}$ \\
\hline C30 & $0.7935(8)$ & $0.5993(7)$ & $0.3783(5)$ & $0.145(4)^{*}$ \\
\hline C31 & $0.2726(3)$ & $0.0691(3)$ & $0.1683(2)$ & $0.0334(8)^{*}$ \\
\hline C32 & $0.2834(3)$ & $-0.0188(3)$ & $0.1038(2)$ & $0.0339(8)^{*}$ \\
\hline C33 & $0.2227(4)$ & $-0.1123(3)$ & $0.1143(2)$ & $0.0397(8)^{*}$ \\
\hline C34 & $0.1577(4)$ & $-0.1194(3)$ & $0.1860(3)$ & $0.0440(9) *$ \\
\hline C35 & $0.1485(4)$ & $-0.0335(3)$ & $0.2514(3)$ & $0.0436(9) *$ \\
\hline C36 & $0.2055(3)$ & $0.0586(3)$ & $0.2386(2)$ & $0.0394(8)^{*}$ \\
\hline
\end{tabular}


Table 2. Atomic Coordinates and Displacement Parameters (continued)

$\begin{array}{lllll}\text { Atom } & x & y & z & U_{\mathrm{eq}}, \AA^{2} \\ \text { C37 } & 0.0798(4) & -0.0392(4) & 0.3322(3) & 0.0558(11)^{*} \\ \text { C38A }^{a} & 0.0457(9) & -0.1463(7) & 0.3407(6) & 0.071(2) \\ \text { C39A }^{a} & 0.1775(9) & 0.0292(7) & 0.4174(6) & 0.079(3) \\ \text { C40A }^{a} & -0.0272(11) & -0.0045(9) & 0.3358(8) & 0.091(3) \\ \text { C38B }^{b} & 0.1183(19) & -0.0935(15) & 0.3762(13) & 0.101(5) \\ \text { C39B } & 0.0846(17) & 0.0697(13) & 0.3954(11) & 0.093(5) \\ \text { C40B } & -0.0710(11) & -0.0794(10) & 0.3015(8) & 0.059(3) \\ \text { C41 } & 0.2228(4) & 0.2151(3) & 0.1498(2) & 0.0396(8)^{*} \\ \text { C42 } & 0.1398(4) & 0.1727(3) & 0.0697(3) & 0.0424(9)^{*} \\ \text { C43 } & 0.0401(4) & 0.2064(4) & 0.0629(3) & 0.0550(11)^{*} \\ \text { C44 } & 0.0253(5) & 0.2837(4) & 0.1342(3) & 0.0626(12)^{*} \\ \text { C45 } & 0.1073(4) & 0.3302(4) & 0.2131(3) & 0.0574(11)^{*} \\ \text { C46 } & 0.2043(4) & 0.2932(3) & 0.2184(3) & 0.0471(10)^{*} \\ \text { C47 } & 0.0968(6) & 0.4197(4) & 0.2902(4) & 0.0780(16)^{*} \\ \text { C48 } & 0.1163(13) & 0.4073(8) & 0.3759(5) & 0.185(6)^{*} \\ \text { C49 } & 0.1912(12) & 0.5158(6) & 0.2849(7) & 0.175(5)^{*} \\ \text { C50 } & -0.0353(9) & 0.4258(8) & 0.2842(7) & 0.185(5)^{*} \\ \text { C51 } & 0.5805(6) & 0.2674(4) & -0.1151(4) & 0.0792(16)^{*} \\ \text { C52 } & 0.6297(7) & 0.2624(6) & -0.2043(6) & 0.105(2)^{*} \\ \text { C53 } & 0.5527(8) & 0.1614(7) & -0.2668(5) & 0.116(3)^{*} \\ \text { C54 } & 0.4603(10) & 0.1125(7) & -0.2215(4) & 0.155(4)^{*}\end{array}$

(b) solvent n-hexane atoms

$\begin{array}{lllcc}\text { Atom } & x & y & z & U_{\text {eq }}, \AA^{2} \\ {\mathrm{C} 1 \mathrm{~S}^{c}}^{c} & -0.0590(10) & 0.4524(4) & -0.0064(14) & 0.178(5) \\ \mathrm{C}^{\mathrm{S}} \mathrm{S}^{c} & -0.1597(9) & 0.4836(14) & 0.0486(12) & 0.178(5) \\ \mathrm{C}^{c} \mathrm{~S}^{c} & -0.0989(18) & 0.5927(13) & 0.1165(12) & 0.178(5)\end{array}$

Anisotropically-refined atoms are marked with an asterisk (*). The form of the anisotropic displacement parameter is: $\exp \left[-2 \pi^{2}\left(h^{2} a^{* 2} U_{11}+k^{2} b^{* 2} U_{22}+l^{2} c^{* 2} U_{33}+2 k l b^{*} c^{*} U_{23}\right.\right.$ $\left.+2 h l a^{*} c^{*} U_{13}+2 h k a^{*} b^{*} U_{12}\right)$ ]. ${ }^{a}$ Refined with an occupancy factor of 0.6. ${ }^{b}$ Refined with an occupancy factor of 0.4. ${ }^{c}$ Carbon atoms of the solvent n-hexane molecule were refined with an occupancy factor of 0.5 and a common isotropic displacement parameter. 
Table 3. Selected Interatomic Distances ( $\AA$ )

$\begin{array}{llllll}\text { Atom1 } & \text { Atom2 } & \text { Distance } & \text { Atom1 } & \text { Atom2 } & \text { Distance } \\ \mathrm{Na} & \mathrm{O} 1 & 2.328(3) & \mathrm{C} 22 & \mathrm{C} 23 & 1.384(6) \\ \mathrm{Na} & \mathrm{O} 2 & 2.473(3) & \mathrm{C} 23 & \mathrm{C} 24 & 1.380(7) \\ \mathrm{Na} & \mathrm{O} 3 & 2.270(3) & \mathrm{C} 24 & \mathrm{C} 25 & 1.395(6) \\ \mathrm{Na} & \mathrm{O} 4 & 2.719(3) & \mathrm{C} 25 & \mathrm{C} 26 & 1.396(6) \\ \mathrm{Na} & \mathrm{O} 5 & 2.350(3) & \mathrm{C} 25 & \mathrm{C} 27 & 1.527(6) \\ \mathrm{Na} & \mathrm{C} 1 & 2.862(4)^{\dagger} & \mathrm{C} 27 & \mathrm{C} 28 & 1.538(9) \\ \mathrm{Na} & \mathrm{C} 2 & 2.906(4)^{\dagger} & \mathrm{C} 27 & \mathrm{C} 29 & 1.529(8) \\ \mathrm{O} 1 & \mathrm{C} 12 & 1.339(4) & \mathrm{C} 27 & \mathrm{C} 30 & 1.481(8) \\ \mathrm{O} 2 & \mathrm{C} 3 & 1.425(5) & \mathrm{C} 31 & \mathrm{C} 32 & 1.415(5) \\ \mathrm{O} 2 & \mathrm{C} 22 & 1.371(5) & \mathrm{C} 31 & \mathrm{C} 36 & 1.389(5) \\ \mathrm{O} 3 & \mathrm{C} 32 & 1.326(4) & \mathrm{C} 32 & \mathrm{C} 33 & 1.396(5) \\ \mathrm{O} 4 & \mathrm{C} 4 & 1.435(5) & \mathrm{C} 33 & \mathrm{C} 34 & 1.386(5) \\ \mathrm{O} 4 & \mathrm{C} 42 & 1.371(5) & \mathrm{C} 34 & \mathrm{C} 35 & 1.394(6) \\ \mathrm{O} 5 & \mathrm{C} 51 & 1.422(6) & \mathrm{C} 35 & \mathrm{C} 36 & 1.388(5) \\ \mathrm{O} 5 & \mathrm{C} 54 & 1.399(7) & \mathrm{C} 35 & \mathrm{C} 37 & 1.530(5) \\ \mathrm{C} 1 & \mathrm{C} 2 & 1.340(5) & \mathrm{C} 37 & \mathrm{C} 38 \mathrm{~A} & 1.558(10) \\ \mathrm{C} 1 & \mathrm{C} 11 & 1.500(5) & \mathrm{C} 37 & \mathrm{C} 39 \mathrm{~A} & 1.558(10) \\ \mathrm{C} 1 & \mathrm{C} 21 & 1.503(5) & \mathrm{C} 37 & \mathrm{C} 40 \mathrm{~A} & 1.448(11) \\ \mathrm{C} 2 & \mathrm{C} 31 & 1.507(5) & \mathrm{C} 37 & \mathrm{C} 38 \mathrm{~B} & 1.385(17) \\ \mathrm{C} 2 & \mathrm{C} 41 & 1.490(5) & \mathrm{C} 37 & \mathrm{C} 39 \mathrm{~B} & 1.581(17) \\ \mathrm{C} 11 & \mathrm{C} 12 & 1.406(5) & \mathrm{C} 37 & \mathrm{C} 40 \mathrm{~B} & 1.613(12) \\ \mathrm{C} 11 & \mathrm{C} 16 & 1.396(5) & \mathrm{C} 41 & \mathrm{C} 42 & 1.405(5) \\ \mathrm{C} 12 & \mathrm{C} 13 & 1.390(6) & \mathrm{C} 41 & \mathrm{C} 46 & 1.385(5) \\ \mathrm{C} 13 & \mathrm{C} 14 & 1.383(6) & \mathrm{C} 42 & \mathrm{C} 43 & 1.383(6) \\ \mathrm{C} 14 & \mathrm{C} 15 & 1.394(6) & \mathrm{C} 43 & \mathrm{C} 44 & 1.385(7) \\ \mathrm{C} 15 & \mathrm{C} 16 & 1.391(6) & \mathrm{C} 44 & \mathrm{C} 45 & 1.383(7) \\ \mathrm{C} 15 & \mathrm{C} 17 & 1.532(6) & \mathrm{C} 45 & \mathrm{C} 46 & 1.385(6) \\ \mathrm{C} 17 & \mathrm{C} 18 \mathrm{~A} & 1.471(12) & \mathrm{C} 45 & \mathrm{C} 47 & 1.526(7) \\ \mathrm{C} 17 & \mathrm{C} 19 \mathrm{~A} & 1.584(12) & \mathrm{C} 47 & \mathrm{C} 48 & 1.470(10) \\ \mathrm{C} 17 & \mathrm{C} 20 \mathrm{~A} & 1.547(12) & \mathrm{C} 47 & \mathrm{C} 49 & 1.501(10) \\ \mathrm{C} 17 & \mathrm{C} 18 \mathrm{~B} & 1.631(16) & \mathrm{C} 50 & 1.520(10) \\ \mathrm{C} 17 & \mathrm{C} 19 \mathrm{~B} & 1.496(16) & \mathrm{C} 52 & 1.529(9) \\ \mathrm{C} 17 & \mathrm{C} 20 \mathrm{~B} & 1.465(17) & \mathrm{C} 53 & 1.464(11) \\ \mathrm{C} 21 & \mathrm{C} 22 & 1.400(5) & & & 1.416(10) \\ \mathrm{C} 21 & \mathrm{C} 26 & 1.395(5) & & & \\ \mathrm{N} & & & & & \end{array}$

$\dagger$ Nonbonded distance. 
Table 4. Selected Interatomic Angles (deg)

\begin{tabular}{|c|c|c|c|c|c|c|c|}
\hline Atom1 & Atom2 & Atom3 & Angle & Atom1 & Atom2 & Atom3 & Angle \\
\hline $\mathrm{O} 1$ & $\mathrm{Na}$ & $\mathrm{O} 2$ & $101.23(10)$ & $\mathrm{Na}$ & $\mathrm{C} 2$ & $\mathrm{C} 41$ & $104.5(2)$ \\
\hline $\mathrm{O} 1$ & $\mathrm{Na}$ & $\mathrm{O} 3$ & $88.45(10)$ & $\mathrm{C} 1$ & $\mathrm{C} 2$ & $\mathrm{C} 31$ & $123.9(3)$ \\
\hline $\mathrm{O} 1$ & $\mathrm{Na}$ & O4 & $148.52(10)$ & $\mathrm{C} 1$ & $\mathrm{C} 2$ & C41 & 122.1(3) \\
\hline $\mathrm{O} 1$ & $\mathrm{Na}$ & O5 & $97.76(13)$ & $\mathrm{C} 31$ & $\mathrm{C} 2$ & C41 & 113.4(3) \\
\hline $\mathrm{O} 1$ & $\mathrm{Na}$ & $\mathrm{C} 1$ & $66.11(10)$ & $\mathrm{C} 1$ & C11 & $\mathrm{C} 12$ & $120.9(3)$ \\
\hline $\mathrm{O} 1$ & $\mathrm{Na}$ & $\mathrm{C} 2$ & $89.64(10)$ & $\mathrm{C} 1$ & $\mathrm{C} 11$ & $\mathrm{C} 16$ & 119.1(3) \\
\hline $\mathrm{O} 2$ & $\mathrm{Na}$ & $\mathrm{O} 3$ & $135.50(11)$ & $\mathrm{C} 12$ & C11 & $\mathrm{C} 16$ & 119.9(3) \\
\hline $\mathrm{O} 2$ & $\mathrm{Na}$ & $\mathrm{O} 4$ & $71.51(10)$ & $\mathrm{O} 1$ & $\mathrm{C} 12$ & $\mathrm{C} 11$ & $120.7(3)$ \\
\hline $\mathrm{O} 2$ & $\mathrm{Na}$ & O5 & $78.33(12)$ & O1 & $\mathrm{C} 12$ & $\mathrm{C} 13$ & 122.1(3) \\
\hline $\mathrm{O} 2$ & $\mathrm{Na}$ & $\mathrm{C} 1$ & $64.30(10)$ & $\mathrm{C} 11$ & $\mathrm{C} 12$ & $\mathrm{C} 13$ & 117.1(3) \\
\hline $\mathrm{O} 2$ & $\mathrm{Na}$ & $\mathrm{C} 2$ & $69.01(10)$ & $\mathrm{C} 12$ & $\mathrm{C} 13$ & $\mathrm{C} 14$ & 122.1(4) \\
\hline $\mathrm{O} 3$ & $\mathrm{Na}$ & $\mathrm{O} 4$ & $77.84(10)$ & $\mathrm{C} 13$ & C14 & $\mathrm{C} 15$ & $121.8(4)$ \\
\hline $\mathrm{O} 3$ & $\mathrm{Na}$ & O5 & $143.82(13)$ & $\mathrm{C} 14$ & $\mathrm{C} 15$ & $\mathrm{C} 16$ & 116.1(4) \\
\hline $\mathrm{O} 3$ & $\mathrm{Na}$ & $\mathrm{C} 1$ & $81.21(10)$ & $\mathrm{C} 14$ & $\mathrm{C} 15$ & $\mathrm{C} 17$ & $122.6(4)$ \\
\hline $\mathrm{O} 3$ & $\mathrm{Na}$ & $\mathrm{C} 2$ & $67.72(10)$ & $\mathrm{C} 16$ & $\mathrm{C} 15$ & $\mathrm{C} 17$ & 121.2(4) \\
\hline $\mathrm{O} 4$ & $\mathrm{Na}$ & O5 & $110.08(13)$ & $\mathrm{C} 11$ & $\mathrm{C} 16$ & $\mathrm{C} 15$ & $123.0(4)$ \\
\hline $\mathrm{O} 4$ & $\mathrm{Na}$ & $\mathrm{C} 1$ & $83.71(10)$ & $\mathrm{C} 15$ & $\mathrm{C} 17$ & C18A & $116.9(6)$ \\
\hline $\mathrm{O} 4$ & $\mathrm{Na}$ & $\mathrm{C} 2$ & $58.99(9)$ & $\mathrm{C} 15$ & $\mathrm{C} 17$ & C19A & $111.2(6)$ \\
\hline O5 & $\mathrm{Na}$ & $\mathrm{C} 1$ & $133.80(13)$ & $\mathrm{C} 15$ & $\mathrm{C} 17$ & $\mathrm{C} 20 \mathrm{~A}$ & $112.2(6)$ \\
\hline O5 & $\mathrm{Na}$ & $\mathrm{C} 2$ & $147.32(13)$ & $\mathrm{C} 15$ & $\mathrm{C} 17$ & C18B & $106.4(7)$ \\
\hline $\mathrm{C} 1$ & $\mathrm{Na}$ & $\mathrm{C} 2$ & $26.85(10)$ & $\mathrm{C} 15$ & $\mathrm{C} 17$ & $\mathrm{C} 19 \mathrm{~B}$ & 107.6(7) \\
\hline $\mathrm{Na}$ & $\mathrm{O} 1$ & $\mathrm{C} 12$ & $119.4(2)$ & $\mathrm{C} 15$ & $\mathrm{C} 17$ & $\mathrm{C} 20 \mathrm{~B}$ & $112.3(8)$ \\
\hline $\mathrm{Na}$ & $\mathrm{O} 2$ & $\mathrm{C} 3$ & $121.7(3)$ & C18A & $\mathrm{C} 17$ & C19A & 104.1(7) \\
\hline $\mathrm{Na}$ & $\mathrm{O} 2$ & $\mathrm{C} 22$ & $121.8(2)$ & C18A & $\mathrm{C} 17$ & $\mathrm{C} 20 \mathrm{~A}$ & $108.7(8)$ \\
\hline $\mathrm{C} 3$ & $\mathrm{O} 2$ & $\mathrm{C} 22$ & $116.5(3)$ & C19A & $\mathrm{C} 17$ & $\mathrm{C} 20 \mathrm{~A}$ & $102.5(7)$ \\
\hline $\mathrm{Na}$ & $\mathrm{O} 3$ & C32 & $127.0(2)$ & $\mathrm{C} 18 \mathrm{~B}$ & $\mathrm{C} 17$ & C19B & $106.5(9)$ \\
\hline $\mathrm{Na}$ & $\mathrm{O} 4$ & $\mathrm{C} 4$ & $116.5(2)$ & C18B & $\mathrm{C} 17$ & C20B & $107.4(10)$ \\
\hline $\mathrm{Na}$ & $\mathrm{O} 4$ & $\mathrm{C} 42$ & $117.4(2)$ & C19B & $\mathrm{C} 17$ & C20B & 116.1(10) \\
\hline $\mathrm{C} 4$ & $\mathrm{O} 4$ & $\mathrm{C} 42$ & 117.3(3) & $\mathrm{C} 1$ & $\mathrm{C} 21$ & $\mathrm{C} 22$ & $123.6(3)$ \\
\hline $\mathrm{Na}$ & O5 & $\mathrm{C} 51$ & $118.0(3)$ & $\mathrm{C} 1$ & $\mathrm{C} 21$ & $\mathrm{C} 26$ & $118.5(3)$ \\
\hline $\mathrm{Na}$ & O5 & C54 & $128.4(4)$ & $\mathrm{C} 22$ & $\mathrm{C} 21$ & $\mathrm{C} 26$ & $117.7(3)$ \\
\hline C51 & O5 & C54 & $110.2(5)$ & $\mathrm{O} 2$ & $\mathrm{C} 22$ & $\mathrm{C} 21$ & $116.9(3)$ \\
\hline $\mathrm{Na}$ & $\mathrm{C} 1$ & $\mathrm{C} 2$ & $78.4(2)$ & $\mathrm{O} 2$ & $\mathrm{C} 22$ & $\mathrm{C} 23$ & $123.1(4)$ \\
\hline $\mathrm{Na}$ & $\mathrm{C} 1$ & $\mathrm{C} 11$ & $98.2(2)$ & $\mathrm{C} 21$ & $\mathrm{C} 22$ & $\mathrm{C} 23$ & $120.0(4)$ \\
\hline $\mathrm{Na}$ & $\mathrm{C} 1$ & $\mathrm{C} 21$ & $101.2(2)$ & $\mathrm{C} 22$ & $\mathrm{C} 23$ & $\mathrm{C} 24$ & $120.8(4)$ \\
\hline $\mathrm{C} 2$ & $\mathrm{C} 1$ & $\mathrm{C} 11$ & $122.2(3)$ & $\mathrm{C} 23$ & $\mathrm{C} 24$ & $\mathrm{C} 25$ & $121.4(4)$ \\
\hline $\mathrm{C} 2$ & $\mathrm{C} 1$ & $\mathrm{C} 21$ & 121.3(3) & $\mathrm{C} 24$ & $\mathrm{C} 25$ & $\mathrm{C} 26$ & $116.7(4)$ \\
\hline $\mathrm{C} 11$ & $\mathrm{C} 1$ & $\mathrm{C} 21$ & $115.9(3)$ & $\mathrm{C} 24$ & $\mathrm{C} 25$ & $\mathrm{C} 27$ & $122.3(4)$ \\
\hline $\mathrm{Na}$ & $\mathrm{C} 2$ & $\mathrm{C} 1$ & $74.7(2)$ & $\mathrm{C} 26$ & $\mathrm{C} 25$ & $\mathrm{C} 27$ & 121.0(4) \\
\hline $\mathrm{Na}$ & $\mathrm{C} 2$ & $\mathrm{C} 31$ & $99.7(2)$ & $\mathrm{C} 21$ & $\mathrm{C} 26$ & $\mathrm{C} 25$ & $123.3(4)$ \\
\hline
\end{tabular}


Table 4. Selected Interatomic Angles (continued)

$\begin{array}{llllllll}\text { Atom1 } & \text { Atom2 } & \text { Atom3 } & \text { Angle } & \text { Atom1 } & \text { Atom2 } & \text { Atom3 } & \text { Angle } \\ \text { C25 } & \text { C27 } & \text { C28 } & 109.4(4) & \text { C39A } & \text { C37 } & \text { C40A } & 109.1(7) \\ \text { C25 } & \text { C27 } & \text { C29 } & 112.2(4) & \text { C38B } & \text { C37 } & \text { C39B } & 113.7(11) \\ \text { C25 } & \text { C27 } & \text { C30 } & 110.3(4) & \text { C38B } & \text { C37 } & \text { C40B } & 114.8(10) \\ \text { C28 } & \text { C27 } & \text { C29 } & 103.8(5) & \text { C39B } & \text { C37 } & \text { C40B } & 93.8(8) \\ \text { C28 } & \text { C27 } & \text { C30 } & 110.3(6) & \text { C2 } & \text { C41 } & \text { C42 } & 121.1(3) \\ \text { C29 } & \text { C27 } & \text { C30 } & 110.7(6) & \text { C2 } & \text { C41 } & \text { C46 } & 120.8(3) \\ \text { C2 } & \text { C31 } & \text { C32 } & 123.4(3) & \text { C42 } & \text { C41 } & \text { C46 } & 118.0(4) \\ \text { C2 } & \text { C31 } & \text { C36 } & 117.4(3) & \text { O4 } & \text { C42 } & \text { C41 } & 115.8(3) \\ \text { C32 } & \text { C31 } & \text { C36 } & 119.1(3) & \text { O4 } & \text { C42 } & \text { C43 } & 124.5(4) \\ \text { O3 } & \text { C32 } & \text { C31 } & 121.0(3) & \text { C41 } & \text { C42 } & \text { C43 } & 119.7(4) \\ \text { O3 } & \text { C32 } & \text { C33 } & 121.9(3) & \text { C42 } & \text { C43 } & \text { C44 } & 119.7(4) \\ \text { C31 } & \text { C32 } & \text { C33 } & 117.1(3) & \text { C43 } & \text { C44 } & \text { C45 } & 122.6(4) \\ \text { C32 } & \text { C33 } & \text { C34 } & 122.0(3) & \text { C44 } & \text { C45 } & \text { C46 } & 116.2(4) \\ \text { C33 } & \text { C34 } & \text { C35 } & 121.8(3) & \text { C44 } & \text { C45 } & \text { C47 } & 123.2(4) \\ \text { C34 } & \text { C35 } & \text { C36 } & 115.7(3) & \text { C46 } & \text { C45 } & \text { C47 } & 120.6(4) \\ \text { C34 } & \text { C35 } & \text { C37 } & 122.7(4) & \text { C41 } & \text { C46 } & \text { C45 } & 123.7(4) \\ \text { C36 } & \text { C35 } & \text { C37 } & 121.6(4) & \text { C45 } & \text { C47 } & \text { C48 } & 111.0(5) \\ \text { C31 } & \text { C36 } & \text { C35 } & 124.2(3) & \text { C45 } & \text { C47 } & \text { C49 } & 107.6(5) \\ \text { C35 } & \text { C37 } & \text { C38A } & 111.8(5) & \text { C45 } & \text { C47 } & \text { C50 } & 111.4(5) \\ \text { C35 } & \text { C37 } & \text { C39A } & 107.7(5) & \text { C48 } & \text { C47 } & \text { C49 } & 112.6(8) \\ \text { C35 } & \text { C37 } & \text { C40A } & 112.8(5) & \text { C48 } & \text { C47 } & \text { C50 } & 105.3(8) \\ \text { C35 } & \text { C37 } & \text { C38B } & 112.7(9) & \text { C49 } & \text { C47 } & \text { C50 } & 108.9(8) \\ \text { C35 } & \text { C37 } & \text { C39B } & 111.7(7) & \text { O5 } & \text { C51 } & \text { C52 } & 106.2(5) \\ \text { C35 } & \text { C37 } & \text { C40B } & 108.6(5) & \text { C51 } & \text { C52 } & \text { C53 } & 105.1(5) \\ \text { C38A } & \text { C37 } & \text { C39A } & 101.3(6) & \text { C52 } & \text { C53 } & \text { C54 } & 108.4(6) \\ \text { C38A } & \text { C37 } & \text { C40A } & 113.3(6) & \text { O5 } & \text { C54 } & \text { C53 } & 110.1(7)\end{array}$


Table 5. Anisotropic Displacement Parameters $\left(U_{\mathrm{ij}}, \AA^{2}\right)$

\begin{tabular}{|c|c|c|c|c|c|c|}
\hline Atom & $U_{11}$ & $U_{22}$ & $U_{33}$ & $U_{23}$ & $U_{13}$ & $U_{12}$ \\
\hline $\mathrm{Na}$ & $0.0617(10)$ & $0.0447(9)$ & $0.0346(8)$ & $0.0178(7)$ & $0.0081(7)$ & $0.0126(7)$ \\
\hline $\mathrm{O} 1$ & $0.0506(15)$ & $0.0379(14)$ & $0.0307(13)$ & $0.0113(11)$ & $0.0138(11)$ & $0.0122(12)$ \\
\hline $\mathrm{O} 2$ & $0.0674(18)$ & $0.0435(16)$ & $0.0364(15)$ & $0.0175(13)$ & $0.0021(13)$ & $0.0154(14)$ \\
\hline $\mathrm{O} 3$ & $0.0552(16)$ & $0.0388(14)$ & $0.0326(14)$ & $0.0128(11)$ & $0.0181(12)$ & $0.0173(12)$ \\
\hline $\mathrm{O} 4$ & $0.0503(16)$ & $0.0500(16)$ & $0.0363(15)$ & $0.0103(12)$ & $-0.0009(12)$ & $0.0171(13)$ \\
\hline $\mathrm{O} 5$ & $0.103(3)$ & $0.063(2)$ & $0.0476(19)$ & $0.0266(16)$ & $0.0197(18)$ & $0.0136(19)$ \\
\hline $\mathrm{C} 1$ & $0.045(2)$ & $0.0333(19)$ & $0.0258(17)$ & $0.0096(14)$ & $0.0094(15)$ & $0.0136(16)$ \\
\hline $\mathrm{C} 2$ & $0.044(2)$ & $0.0371(19)$ & $0.0243(17)$ & $0.0095(15)$ & $0.0071(15)$ & $0.0160(16)$ \\
\hline $\mathrm{C} 3$ & $0.074(3)$ & $0.062(3)$ & $0.053(3)$ & $0.031(2)$ & $-0.005(2)$ & $0.016(2)$ \\
\hline $\mathrm{C} 4$ & $0.060(3)$ & $0.059(3)$ & $0.043(2)$ & $0.014(2)$ & $-0.007(2)$ & $0.016(2)$ \\
\hline $\mathrm{C} 11$ & $0.0393(19)$ & $0.0337(19)$ & $0.0316(19)$ & $0.0093(15)$ & $0.0058(15)$ & $0.0108(15)$ \\
\hline C12 & $0.0373(19)$ & $0.0314(18)$ & $0.0347(19)$ & $0.0086(15)$ & $0.0074(15)$ & $0.0055(15)$ \\
\hline C13 & $0.047(2)$ & $0.055(3)$ & $0.047(2)$ & $0.013(2)$ & $0.0127(19)$ & $0.023(2)$ \\
\hline $\mathrm{C} 14$ & $0.060(3)$ & $0.077(3)$ & $0.047(3)$ & $0.016(2)$ & $0.006(2)$ & $0.041(2)$ \\
\hline $\mathrm{C} 15$ & $0.062(3)$ & $0.059(3)$ & $0.041(2)$ & $0.014(2)$ & $0.0057(19)$ & $0.032(2)$ \\
\hline $\mathrm{C} 16$ & $0.049(2)$ & $0.044(2)$ & $0.035(2)$ & $0.0127(17)$ & $0.0118(17)$ & $0.0226(18)$ \\
\hline $\mathrm{C} 17$ & $0.096(4)$ & $0.095(4)$ & $0.041(2)$ & $0.021(3)$ & $0.006(2)$ & $0.059(3)$ \\
\hline $\mathrm{C} 21$ & $0.046(2)$ & $0.037(2)$ & $0.035(2)$ & $0.0142(16)$ & $0.0110(16)$ & $0.0147(17)$ \\
\hline $\mathrm{C} 22$ & $0.057(2)$ & $0.039(2)$ & $0.039(2)$ & $0.0171(18)$ & $0.0076(18)$ & $0.0113(18)$ \\
\hline $\mathrm{C} 23$ & $0.082(3)$ & $0.047(3)$ & $0.052(3)$ & $0.028(2)$ & $0.006(2)$ & $0.016(2)$ \\
\hline $\mathrm{C} 24$ & $0.083(3)$ & $0.037(2)$ & $0.061(3)$ & $0.021(2)$ & $0.005(2)$ & $0.013(2)$ \\
\hline $\mathrm{C} 25$ & $0.057(3)$ & $0.043(2)$ & $0.051(3)$ & $0.013(2)$ & $0.009(2)$ & $0.012(2)$ \\
\hline $\mathrm{C} 26$ & $0.049(2)$ & $0.042(2)$ & $0.041(2)$ & $0.0150(18)$ & $0.0098(18)$ & $0.0159(18)$ \\
\hline $\mathrm{C} 27$ & $0.066(3)$ & $0.044(2)$ & $0.057(3)$ & $0.008(2)$ & $0.001(2)$ & $0.011(2)$ \\
\hline $\mathrm{C} 28$ & $0.129(6)$ & $0.111(6)$ & $0.065(4)$ & $-0.002(4)$ & $0.014(4)$ & $0.018(5)$ \\
\hline C29 & $0.164(7)$ & $0.058(4)$ & $0.093(5)$ & $-0.012(3)$ & $-0.035(5)$ & $0.037(4)$ \\
\hline $\mathrm{C} 30$ & $0.109(6)$ & $0.153(8)$ & $0.112(6)$ & $-0.047(5)$ & $-0.036(5)$ & $0.063(6)$ \\
\hline C31 & $0.0344(18)$ & $0.0364(19)$ & $0.0286(18)$ & $0.0124(15)$ & $0.0036(14)$ & $0.0102(15)$ \\
\hline C32 & $0.0356(18)$ & $0.0368(19)$ & $0.0291(18)$ & $0.0128(15)$ & $0.0051(14)$ & $0.0113(15)$ \\
\hline C33 & $0.046(2)$ & $0.038(2)$ & $0.035(2)$ & $0.0122(16)$ & $0.0084(16)$ & $0.0145(17)$ \\
\hline C34 & $0.049(2)$ & $0.044(2)$ & $0.042(2)$ & $0.0242(18)$ & $0.0098(18)$ & $0.0105(18)$ \\
\hline $\mathrm{C} 35$ & $0.044(2)$ & $0.052(2)$ & $0.037(2)$ & $0.0205(18)$ & $0.0111(17)$ & $0.0150(18)$ \\
\hline C36 & $0.044(2)$ & $0.044(2)$ & $0.0307(19)$ & $0.0125(16)$ & $0.0103(16)$ & $0.0174(17)$ \\
\hline C37 & $0.066(3)$ & $0.063(3)$ & $0.041(2)$ & $0.025(2)$ & $0.022(2)$ & $0.017(2)$ \\
\hline $\mathrm{C} 41$ & $0.043(2)$ & $0.040(2)$ & $0.038(2)$ & $0.0172(17)$ & $0.0090(16)$ & $0.0140(17)$ \\
\hline $\mathrm{C} 42$ & $0.041(2)$ & $0.046(2)$ & $0.042(2)$ & $0.0184(18)$ & $0.0064(17)$ & $0.0143(17)$ \\
\hline $\mathrm{C} 43$ & $0.049(2)$ & $0.063(3)$ & $0.059(3)$ & $0.027(2)$ & $0.003(2)$ & $0.022(2)$ \\
\hline $\mathrm{C} 44$ & $0.058(3)$ & $0.074(3)$ & $0.075(3)$ & $0.035(3)$ & $0.018(2)$ & $0.040(3)$ \\
\hline $\mathrm{C} 45$ & $0.062(3)$ & $0.057(3)$ & $0.064(3)$ & $0.023(2)$ & $0.017(2)$ & $0.032(2)$ \\
\hline $\mathrm{C} 46$ & $0.052(2)$ & $0.046(2)$ & $0.047(2)$ & $0.0174(19)$ & $0.0129(19)$ & $0.0212(19)$ \\
\hline
\end{tabular}


Table 5. Anisotropic Displacement Parameters (continued)

\begin{tabular}{lllllll} 
Atom & $U_{11}$ & $U_{22}$ & $U_{33}$ & $U_{23}$ & $U_{13}$ & \multicolumn{1}{c}{$U_{12}$} \\
C47 & $0.094(4)$ & $0.070(3)$ & $0.079(4)$ & $0.013(3)$ & $0.017(3)$ & $0.054(3)$ \\
C48 & $0.369(17)$ & $0.186(10)$ & $0.081(5)$ & $0.040(6)$ & $0.094(8)$ & $0.205(12)$ \\
C49 & $0.246(12)$ & $0.062(5)$ & $0.169(9)$ & $0.002(5)$ & $0.088(9)$ & $0.029(6)$ \\
C50 & $0.147(8)$ & $0.170(9)$ & $0.202(11)$ & $-0.039(8)$ & $0.015(7)$ & $0.117(8)$ \\
C51 & $0.089(4)$ & $0.063(3)$ & $0.102(4)$ & $0.046(3)$ & $0.030(3)$ & $0.029(3)$ \\
C52 & $0.121(5)$ & $0.111(5)$ & $0.146(7)$ & $0.098(5)$ & $0.078(5)$ & $0.067(5)$ \\
C53 & $0.144(7)$ & $0.149(8)$ & $0.078(5)$ & $0.057(5)$ & $0.048(5)$ & $0.062(6)$ \\
C54 & $0.195(9)$ & $0.149(7)$ & $0.045(4)$ & $0.017(4)$ & $0.035(5)$ & $-0.021(6)$
\end{tabular}

The form of the anisotropic displacement parameter is:

$\exp \left[-2 \pi^{2}\left(h^{2} a^{* 2} U_{11}+k^{2} b^{* 2} U_{22}+l^{2} c^{* 2} U_{33}+2 k l b^{*} c^{*} U_{23}+2 h l a^{*} c^{*} U_{13}+2 h k a^{*} b^{*} U_{12}\right)\right]$ 
Table 6. Derived Atomic Coordinates and Displacement Parameters for Hydrogen Atoms

\begin{tabular}{|c|c|c|c|c|}
\hline Atom & $x$ & $y$ & $z$ & $U_{\text {eq }}, \AA^{2}$ \\
\hline $\mathrm{H} 3 \mathrm{O}$ & 0.3682 & -0.0735 & 0.0011 & 0.063 \\
\hline $\mathrm{H} 3 \mathrm{~A}$ & 0.2803 & 0.2595 & -0.0741 & 0.075 \\
\hline H3B & 0.3768 & 0.3750 & -0.0377 & 0.075 \\
\hline $\mathrm{H} 3 \mathrm{C}$ & 0.2560 & 0.3413 & 0.0115 & 0.075 \\
\hline $\mathrm{H} 4 \mathrm{~A}$ & 0.1122 & 0.0037 & -0.1230 & 0.068 \\
\hline H4B & 0.0828 & 0.1068 & -0.1045 & 0.068 \\
\hline $\mathrm{H} 4 \mathrm{C}$ & -0.0030 & 0.0201 & -0.0699 & 0.068 \\
\hline H13 & 0.7809 & 0.1193 & 0.1391 & 0.060 \\
\hline H14 & 0.8070 & 0.1282 & 0.2846 & 0.070 \\
\hline H16 & 0.5041 & 0.2161 & 0.3310 & 0.050 \\
\hline $\mathrm{H}_{18 \mathrm{~A}^{a}}$ & 0.6199 & 0.2343 & 0.5431 & 0.113 \\
\hline $\mathrm{H} 18 \mathrm{~B}^{a}$ & 0.5105 & 0.1949 & 0.4614 & 0.113 \\
\hline $\mathrm{H} 18 \mathrm{C}^{a}$ & 0.6211 & 0.3034 & 0.4842 & 0.113 \\
\hline $\mathrm{H}_{19 \mathrm{~A}^{a}}$ & 0.8772 & 0.2215 & 0.4324 & 0.116 \\
\hline $\mathrm{H}^{19 \mathrm{~B}^{a}}$ & 0.8299 & 0.2482 & 0.5282 & 0.116 \\
\hline $\mathrm{H} 19 \mathrm{C}^{a}$ & 0.8380 & 0.3206 & 0.4715 & 0.116 \\
\hline $\mathrm{H} 20 \mathrm{~A}^{a}$ & 0.7103 & 0.0466 & 0.3931 & 0.122 \\
\hline $\mathrm{H} 20 \mathrm{~B}^{a}$ & 0.5697 & 0.0367 & 0.4165 & 0.122 \\
\hline $\mathrm{H} 20 \mathrm{C}^{a}$ & 0.6872 & 0.0908 & 0.4944 & 0.122 \\
\hline $\mathrm{H} 18 \mathrm{D}^{b}$ & 0.4768 & 0.1495 & 0.4330 & 0.102 \\
\hline $\mathrm{H} 18 \mathrm{E}^{b}$ & 0.5461 & 0.1236 & 0.5053 & 0.102 \\
\hline $\mathrm{H} 18 \mathrm{~F}^{b}$ & 0.5134 & 0.0487 & 0.4036 & 0.102 \\
\hline $\mathrm{H} 19 \mathrm{D}^{b}$ & 0.6407 & 0.3173 & 0.4620 & 0.103 \\
\hline $\mathrm{H}_{19 \mathrm{E}^{b}}$ & 0.7891 & 0.3350 & 0.4709 & 0.103 \\
\hline $\mathrm{H}_{19 \mathrm{~F}^{b}}$ & 0.7054 & 0.3000 & 0.5422 & 0.103 \\
\hline $\mathrm{H} 20 \mathrm{D}^{b}$ & 0.7761 & 0.1451 & 0.5028 & 0.112 \\
\hline $\mathrm{H} 20 \mathrm{E}^{b}$ & 0.8509 & 0.1721 & 0.4250 & 0.112 \\
\hline $\mathrm{H} 20 \mathrm{~F}^{b}$ & 0.7375 & 0.0652 & 0.4029 & 0.112 \\
\hline $\mathrm{H} 23$ & 0.4615 & 0.4927 & 0.0814 & 0.072 \\
\hline $\mathrm{H} 24$ & 0.5713 & 0.6166 & 0.2149 & 0.074 \\
\hline H26 & 0.5905 & 0.3971 & 0.3052 & 0.053 \\
\hline $\mathrm{H} 28 \mathrm{~A}$ & 0.6352 & 0.6308 & 0.4975 & 0.140 \\
\hline H28B & 0.5810 & 0.5115 & 0.4395 & 0.140 \\
\hline $\mathrm{H} 28 \mathrm{C}$ & 0.5051 & 0.5835 & 0.4315 & 0.140 \\
\hline H29A & 0.7146 & 0.7568 & 0.4308 & 0.139 \\
\hline H29B & 0.5817 & 0.7085 & 0.3676 & 0.139 \\
\hline $\mathrm{H} 29 \mathrm{C}$ & 0.7117 & 0.7270 & 0.3253 & 0.139 \\
\hline H30A & 0.8376 & 0.6507 & 0.4359 & 0.174 \\
\hline H30B & 0.8403 & 0.6145 & 0.3306 & 0.174 \\
\hline $\mathrm{H} 30 \mathrm{C}$ & 0.7883 & 0.5316 & 0.3774 & 0.174 \\
\hline
\end{tabular}


Table 6. Derived Parameters for Hydrogen Atoms (continued)

\begin{tabular}{|c|c|c|c|c|}
\hline Atom & $x$ & $y$ & $z$ & $U_{\text {eq }}, \AA^{2}$ \\
\hline H33 & 0.2261 & -0.1729 & 0.0710 & 0.048 \\
\hline H34 & 0.1183 & -0.1845 & 0.1907 & 0.053 \\
\hline H36 & 0.1980 & 0.1183 & 0.2807 & 0.047 \\
\hline $\mathrm{H}_{38 \mathrm{~A}^{a}}$ & 0.1215 & -0.1657 & 0.3375 & 0.085 \\
\hline $\mathrm{H}^{2} 8 \mathrm{~B}^{a}$ & -0.0204 & -0.1967 & 0.2921 & 0.085 \\
\hline $\mathrm{H} 38 \mathrm{C}^{a}$ & 0.0147 & -0.1440 & 0.3979 & 0.085 \\
\hline $\mathrm{H}_{39 \mathrm{~A}^{a}}$ & 0.2494 & 0.0054 & 0.4157 & 0.095 \\
\hline $\mathrm{H} 39 \mathrm{~B}^{a}$ & 0.1367 & 0.0251 & 0.4703 & 0.095 \\
\hline $\mathrm{H} 39 \mathrm{C}^{a}$ & 0.2077 & 0.1001 & 0.4196 & 0.095 \\
\hline $\mathrm{H}_{40 \mathrm{~A}^{a}}$ & -0.0675 & -0.0097 & 0.3887 & 0.109 \\
\hline $\mathrm{H}_{40 \mathrm{~B}^{a}}$ & -0.0888 & -0.0471 & 0.2823 & 0.109 \\
\hline $\mathrm{H} 40 \mathrm{C}^{a}$ & 0.0024 & 0.0667 & 0.3389 & 0.109 \\
\hline $\mathrm{H} 38 \mathrm{D}^{b}$ & 0.0725 & -0.0941 & 0.4276 & 0.122 \\
\hline $\mathrm{H} 38 \mathrm{E}^{b}$ & 0.2097 & -0.0610 & 0.3964 & 0.122 \\
\hline $\mathrm{H} 38 \mathrm{~F}^{b}$ & 0.0999 & -0.1637 & 0.3355 & 0.122 \\
\hline $\mathrm{H} 39 \mathrm{D}^{b}$ & 0.0668 & 0.1072 & 0.3595 & 0.112 \\
\hline $\mathrm{H}_{39 \mathrm{E}^{b}}$ & 0.1692 & 0.1076 & 0.4299 & 0.112 \\
\hline $\mathrm{H}_{39 \mathrm{~F}^{b}}$ & 0.0209 & 0.0619 & 0.4361 & 0.112 \\
\hline $\mathrm{H} 40 \mathrm{D}^{b}$ & -0.0918 & -0.0304 & 0.2806 & 0.070 \\
\hline $\mathrm{H} 40 \mathrm{E}^{b}$ & -0.1164 & -0.0865 & 0.3521 & 0.070 \\
\hline $\mathrm{H} 40 \mathrm{~F}^{b}$ & -0.0966 & -0.1458 & 0.2532 & 0.070 \\
\hline $\mathrm{H} 43$ & -0.0178 & 0.1766 & 0.0096 & 0.066 \\
\hline $\mathrm{H} 44$ & -0.0440 & 0.3057 & 0.1287 & 0.075 \\
\hline H46 & 0.2615 & 0.3230 & 0.2721 & 0.057 \\
\hline H48A & 0.1088 & 0.4654 & 0.4242 & 0.222 \\
\hline H48B & 0.2011 & 0.4040 & 0.3844 & 0.222 \\
\hline $\mathrm{H} 48 \mathrm{C}$ & 0.0521 & 0.3441 & 0.3763 & 0.222 \\
\hline H49A & 0.1856 & 0.5748 & 0.3331 & 0.210 \\
\hline H49B & 0.1726 & 0.5216 & 0.2274 & 0.210 \\
\hline $\mathrm{H} 49 \mathrm{C}$ & 0.2770 & 0.5136 & 0.2907 & 0.210 \\
\hline H50A & -0.0385 & 0.4846 & 0.3338 & 0.221 \\
\hline H50B & -0.0977 & 0.3631 & 0.2871 & 0.221 \\
\hline $\mathrm{H} 50 \mathrm{C}$ & -0.0548 & 0.4335 & 0.2277 & 0.221 \\
\hline H51A & 0.6483 & 0.2752 & -0.0706 & 0.095 \\
\hline H51B & 0.5507 & 0.3259 & -0.0923 & 0.095 \\
\hline H52A & 0.6191 & 0.3168 & -0.2234 & 0.126 \\
\hline H52B & 0.7207 & 0.2702 & -0.1999 & 0.126 \\
\hline H53A & 0.5107 & 0.1682 & -0.3179 & 0.140 \\
\hline H53B & 0.6072 & 0.1204 & -0.2898 & 0.140 \\
\hline H54A & 0.3744 & 0.0997 & -0.2490 & 0.186 \\
\hline
\end{tabular}


Table 6. Derived Parameters for Hydrogen Atoms (continued)

$\begin{array}{lcccc}\text { Atom } & x & y & z & U_{\mathrm{eq}}, \AA^{2} \\ \text { H54B } & 0.4672 & 0.0460 & -0.2266 & 0.186 \\ \mathrm{H}^{2} \mathrm{SA}^{c} & -0.0349 & 0.4030 & 0.0129 & 0.214 \\ \mathrm{H}^{c} \mathrm{SB}^{c} & -0.0944 & 0.4191 & -0.0702 & 0.214 \\ \mathrm{H}^{c} \mathrm{SA}^{c} & -0.2296 & 0.4807 & 0.0085 & 0.214 \\ \mathrm{H}^{c} \mathrm{SB}^{c} & -0.1956 & 0.4356 & 0.0802 & 0.214 \\ \mathrm{H}^{c} \mathrm{SA}^{c} & -0.1635 & 0.6127 & 0.1507 & 0.214 \\ \mathrm{H}^{c} \mathrm{SB}^{c} & -0.0631 & 0.6400 & 0.0851 & 0.214 \\ \mathrm{H} 3 S C^{c} & -0.0313 & 0.5949 & 0.1571 & 0.214\end{array}$

${ }^{a}$ Included with an occupancy factor of 0.6. ${ }^{b}$ Includd with an occupancy factor of 0.4. ${ }^{c}$ Included with an occupancy factor of 0.5 . 